\title{
Kerr-de Sitter quasinormal modes via accessory parameter expansion
}

\author{
Fábio Novaes, ${ }^{a}$ Cássio I.S. Marinho, ${ }^{b}$ Máté Lencsés ${ }^{a}$ and Marc Casals ${ }^{b, c}$ \\ ${ }^{a}$ International Institute of Physics, Federal University of Rio Grande do Norte, \\ Campus Universitário, \\ Lagoa Nova, Natal-RN 59078-970, Brazil \\ ${ }^{b}$ Centro Brasileiro de Pesquisas Fúsicas (CBPF), \\ Rio de Janeiro, CEP 22290-180, Brazil \\ ${ }^{c}$ School of Mathematics and Statistics, University College Dublin, \\ Belfield, Dublin 4, Ireland \\ E-mail: fabionsantos@gmail.com, marinho@cbpf.br, \\ matelencses@gmail.com, mcasals@cbpf .br
}

ABSTRACT: Quasinormal modes are characteristic oscillatory modes that control the relaxation of a perturbed physical system back to its equilibrium state. In this work, we calculate QNM frequencies and angular eigenvalues of Kerr-de Sitter black holes using a novel method based on conformal field theory. The spin-field perturbation equations of this background spacetime essentially reduce to two Heun's equations, one for the radial part and one for the angular part. We use the accessory parameter expansion of Heun's equation, obtained via the isomonodromic $\tau$-function, in order to find analytic expansions for the QNM frequencies and angular eigenvalues. The expansion for the frequencies is given as a double series in the rotation parameter $a$ and the extremality parameter $\epsilon=\left(r_{C}-r_{+}\right) / L$, where $L$ is the de Sitter radius and $r_{C}$ and $r_{+}$are the radii of, respectively, the cosmological and event horizons. Specifically, we give the frequency expansion up to order $\epsilon^{2}$ for general $a$, and up to order $\epsilon^{3}$ with the coefficients expanded up to $(a / L)^{3}$. Similarly, the expansion for the angular eigenvalues is given as a series up to $(a \omega)^{3}$ with coefficients expanded for small $a / L$. We verify the new expansion for the frequencies via a numerical analysis and that the expansion for the angular eigenvalues agrees with results in the literature.

Keywords: Black Holes, Classical Theories of Gravity, Conformal Field Theory

ARXIV EPRINT: 1811.11912 


\section{Contents}

1 Introduction 1

2 Kerr-de Sitter black holes $\quad 5$

3 Scattering on Kerr-de Sitter black holes $\quad 8$

3.1 Linear field perturbations 8

3.2 Reduction of radial and angular equations to Heun equations 9

$\begin{array}{lll}3.3 & \text { Kerr-de Sitter connection coefficients and greybody factor } & 12\end{array}$

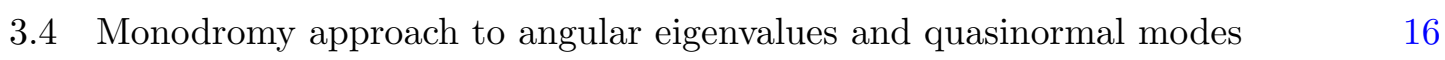

3.5 Quasinormal modes in the rotating Nariai limit 21

4 Accessory parameter expansion $\quad 24$

4.1 How to obtain the APE 25

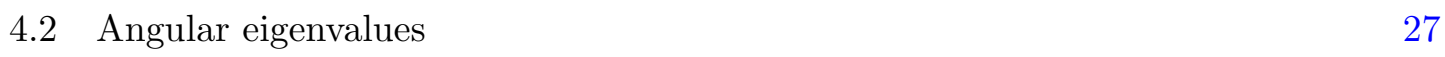

$\begin{array}{lll}4.3 & \text { Quasinormal mode frequencies } & 28\end{array}$

5 Comparison with numerical calculation $\quad 31$

6 Conclusions 31

A Gauge transformations of Fuchsian equations $\quad 35$

$\begin{array}{ll}\text { A.1 Radial master equation } & 37\end{array}$

$\begin{array}{lll}\text { A.2 Angular master equation } & 41\end{array}$

B Alternative derivation of quasinormal mode equation $\quad 42$

C Accessory parameter expansion from isomonodromic $\tau$-function $\quad 43$

D Coefficients of the quasinormal mode expansion $\quad 45$

$\begin{array}{lll}\text { E Coefficients of the angular eigenvalue expansion } & 47\end{array}$

\section{Introduction}

Quasinormal Modes (QNMs) are free oscillatory modes of an open physical system. Contrary to normal modes, QNMs oscillate but also decay exponentially in time. The exponential ringdown of these modes is thus characterized by complex frequencies $\omega_{\mathrm{QNM}}=\omega_{R}+i \omega_{I}$, where $\omega_{R} \in \mathbb{R}$ corresponds to the frequency of the oscillation and $\omega_{I}<0$ sets the timescale of the decay. The study of QNMs and their exponentially-growing counterparts $\left(\omega_{I}>0\right)$ is important for, e.g., the linear stability analysis of background spacetimes (e.g., [1]), to characterize the ringdown stage of a waveform from a black hole binary inspiral [2] and for setting the thermalization scale in holographic gauge theories [3]. For reviews on QNMs, see, e.g., $[4,5]$. 
Black holes are compact objects characterized by the existence of an event horizon. Among the class of stationary and axisymmetric solutions of General Relativity, we can find exact black hole spacetimes. They are classified by their mass $M$, angular momentum $J$, charge $Q$ and by the cosmological constant $\Lambda$. In the following, we use the more convenient angular momentum parameter per unit mass, $a \equiv J / M$, and the de Sitter radius $L \equiv \sqrt{3 / \Lambda}(\Lambda>0)$.

In this paper, we obtain QNMs and angular eigenvalues for the spin- $s$ perturbation equations of four-dimensional Kerr-de Sitter black holes $(\Lambda>0)$. These black holes contain four horizons and we focus on the QNMs obtained from solutions which are valid between the outer event horizon (at radius $r_{+}$) and the cosmological horizon (at radius $r_{C} \geq r_{+}$).

A standard approach for studying massless spin-field perturbations of black hole spacetimes is to write the spin- $s$ field equations using the Newman-Penrose formalism. This formalism enables the decoupling and simplification of the linear field perturbation equations into one single ("master") partial differential equation for any Petrov type D spacetime. In the absence of sources, this master equation is separable into two ordinary differential equations (ODEs) for modes with fixed frequency $\omega$ and azimuthal angular number $m$. This approach was first pursued by Teukolsky for a Kerr black hole [6]. More generally, one can find a master equation for spin $s=0, \pm 1 / 2, \pm 1, \pm 3 / 2, \pm 2$ massless field perturbations of any Petrov type D spacetime [7-10], including Kerr-de Sitter black holes. The associated ODEs are all reducible to Heun equations [10,11]. In this work, we exploit an integrable structure behind Heun equations, deeply related to 2D conformal field theory (CFT), to find QNMs and angular eigenvalues of Kerr-de Sitter black holes.

Heun equations are ODEs with four regular singular points [12]. We can fix these points to be at the values $z=0,1, x, \infty$ of the independent variable, without loss of generality. These equations can then be characterized by the moduli parameter $x$, its local monodromy data and the so-called accessory parameter. The monodromy data governs the behaviour of the analytically continued solutions around the singular points, while the accessory parameter contains global information of the solutions. Heun equations can be formally extended to have an extra apparent singularity, with trivial monodromy. The position of the apparent singularity can then be continuously changed in a precise way to leave unaffected the monodromy data. This is called the isomonodromic deformation of the Heun equation $[13,14]$. Perhaps surprisingly, this deformation is Hamiltonian and integrable. One can use this structure to construct an expansion of the accessory parameter in $x$ with coefficients given in terms of the local and global monodromy data [15, 16]. We call this the Accessory Parameter Expansion (APE), revised in section 4.1. This approach was successfully applied in the context of $2 \mathrm{D}$ conformal field theory in relation to the classical limit of conformal blocks [16] and for 2D conformal mappings from a simply connected planar region to the interior of a circular arc quadrilateral [17]. For other approaches to obtaining the APE, we refer the reader to $[15,18,19]$.

In this work, we use the APE to calculate Kerr-de Sitter QNMs and angular eigenvalues for the first time. In particular, we apply the APE to the case of Kerr-de Sitter black holes near the extremal limit $r_{C} \rightarrow r_{+}$, also called the rotating Nariai limit, about both the upper 
and lower superradiant bounds. ${ }^{1}$ More specifically, we obtain an analytical expansion for the QNM frequencies of the form:

$$
{ }_{s} \omega_{\ell m}=m \Omega_{+}+\epsilon\left[\bar{\omega}_{0}(a)+\bar{\omega}_{1}(a) \epsilon^{1}+\bar{\omega}_{2}(a) \epsilon^{2}+\mathcal{O}\left(\epsilon^{3}\right)\right],
$$

in the extremality parameter $\epsilon \equiv\left(r_{C}-r_{+}\right) / L$, where $\bar{\omega}_{0,1,2}$ are coefficients we determine. Here and in what follows $\Omega_{k}, k=+, C$ denotes the angular velocity of the horizon $r_{k}$, $k=+, C$. We provide this expansion: (i) up to third order in $\epsilon$ with the coefficients expanded up to third order in $a / L$; (ii) up to second order in $\epsilon$ for general $a$. The expansion in eq. (1.1) is about $m \Omega_{+}$(unexpanded in $\epsilon$ ). The expansion about $m \Omega_{C}$ can be obtained by letting $\Omega_{+} \rightarrow \Omega_{C}, \epsilon \rightarrow-\epsilon$ and taking the complex conjugate of the right hand side of eq. (1.1). We thus show, and check numerically, that the lower superradiant bound $\omega=m \Omega_{C}$ is an accumulation point of QNMs, similarly to the upper bound $\omega=m \Omega_{+}$.

To the best of our knowledge, only the term $\bar{\omega}_{0}$ in eq. (1.1) for the specific case of $s=0$ and coupling constant with the Ricci scalar $\xi=0$ had so far been obtained in the literature. It was obtained in [20] by calculating QNMs directly on the rotating Nariai geometry, which is found by a near horizon limit of the Kerr-de Sitter spacetime. Apart from the APE derivation of eq. (1.1), we also calculate QNMs directly in the rotating Nariai geometry in the case of $s=0$ and general $\xi$, thus obtaining an alternative derivation of $\bar{\omega}_{0}$ for this case. Overall, we believe that the expressions we derive for $\bar{\omega}_{0}$ for non-zero spin (as well as for $s=0$ and $\xi \neq 0$ ) and for $\bar{\omega}_{1,2}$ for any spin are new. We have checked our high-order general-spin eq. (1.1) against a numerical analysis that we have carried out using Leaver's method to high accuracy. This method was first used in [21] to obtain Kerr-de Sitter QNMs and angular eigenvalues and our results are consistent with that work.

Using the same APE method, we derive an analytical expansion for the angular eigenvalue

$$
{ }_{s} \lambda_{\ell m}=\sum_{\substack{n, k=0 \\ n+k=3}}^{3} \lambda_{n, k} \alpha^{n}(a \omega)^{k}+\sum_{\substack{n, k=0 \\ n+k=4}}^{4} \mathcal{O}\left(\alpha^{n}(a \omega)^{k}\right)
$$

where $\lambda_{n, k}$ are the coefficients, obtained as a small $\alpha \equiv a / L$ expansion from the APE. For convenience, we will reorganize the explicit coefficients of eq. (1.2) as a finite series

$$
{ }_{s} \lambda_{\ell m}=\sum_{k=0}^{3} \lambda_{\omega, k}(a \omega)^{k}+\mathcal{O}\left((a \omega)^{4}\right)
$$

where the coefficients $\lambda_{\omega, k} \equiv \sum_{n=0}^{3-k} \lambda_{n, k} \alpha^{n}+\mathcal{O}\left(\alpha^{4-k}\right), k=0, \ldots, 3$, are given in appendix E. In the rest of this paper, we will only refer to the coefficients in eq. (1.3).

Kerr-de Sitter angular eigenvalues were first obtained numerically in [9]. Our expansion for the eigenvalues for small $\alpha$ and aw agrees with that already obtained in [11] using a completely different method (and with that in [22] in the Kerr limit $L \rightarrow \infty$ ). Our

\footnotetext{
${ }^{1}$ Superradiance is a scattering phenomenon whereby a field wave extracts rotational energy from a rotating black hole.
} 
eigenvalue derivation here is a proof of principle of the APE, which is extendable to higher orders than what we present here.

Summing up, with our method, we obtain: (i) an angular eigenvalue expansion for small $a \omega$ and $a / L$ consistent with $[11,22]$, and (ii) a new expansion for the QNM frequencies near the rotating Nariai limit. For the reader who is only interested in the final result, the coefficients in the QNM expansion (1.1) are given in eqs. (4.15) and (4.18) and in appendix D, and the coefficients in the eigenvalue expansion (1.3) are given in appendix E.

There exist various other methods for calculating QNM frequencies, from asymptotic analyses to numerical techniques. Ref. [21] provides a numerical calculation of QNMs of Kerr-de Sitter black holes, for modes between $r_{C}$ and $r_{+}$, by using a method originally developed by Leaver $[23,24]$. Their analysis suggests that the QNM frequencies accumulate towards $\omega=m \Omega_{+}$in each of the extremal limits $r_{+} \rightarrow r_{-}$and $r_{+} \rightarrow r_{C}$, where $\Omega_{+}$is the angular velocity of the event horizon $r_{+}$. The frequency $\omega=m \Omega_{+}$is the upper bound of the superradiant regime. For non-extremal (i.e., $r_{+} \neq r_{C}$ ) Kerr-de Sitter black holes, the superradiant regime is $|m| \Omega_{C}<|\omega|<|m| \Omega_{+}$, where $\Omega_{C}$ is the angular velocity of the cosmological horizon $r_{C}$. Other works we found in the literature on QNMs in Kerr-de Sitter are the following: [25] provides an analytic expansion of the QNM with the lowest overtone $(N=0)$ in the double approximation of small $a$ (to linear order) and large- $(\ell+1 / 2)$ (up to order $\left.(\ell+1 / 2)^{-6}\right)$, where $\ell$ is the multipolar number; similarly, [26] provides a BohrSommerfeld type of condition which QNMs for small $a$ must satisfy and which could be solved numerically, being better suited for larger $\ell$; within the context of the so-called strong cosmic censorship conjecture, [27] obtained an analytic expression for the QNMs for $\ell=|m| \gg 1$ and complement it with a numerical study.

We finish the introduction by outlining the contents of this paper. We describe the geometry and configuration space of the Kerr-de Sitter black hole in section 2. In section 3, we review the spin-field master equation in Kerr-de Sitter and the scattering problem. We express the product of scattering coefficients, the so-called greybody factor, in terms of the monodromies of the radial ODE. We then describe the monodromy conditions for angular eigenvalues and QNMs. We finish section 3 by obtaining QNMs directly in the rotating Nariai geometry [20] and obtaining the same result from the full Heun equation in Kerrde Sitter under the appropriate limits. In section 4, we review how to obtain the APE via the isomonodromic $\tau$-function, first proposed in [28]. In subsections 4.2 and 4.3, we apply the APE around $\epsilon=0$ to obtain expansions for the angular eigenvalues and QNMs. In section 5, we compare our analytic results with a numerical calculation using Leaver's method. In section 6 we present our conclusions and potential future developments.

We also include complementary sections with more details on our results. In appendix A, we introduce the gauge transformations of Fuchsian equations used to reduce the master equations to Heun equations. In appendix B, we present an alternative derivation of the leading order QNM equation. In appendix C, we review how to obtain the APE from the isomonodromic $\tau$-function as in [16], with the crucial modification of expanding the monodromies in $\epsilon$. Finally, in appendices D and $\mathrm{E}$ we display the coefficients of the QNM expansion and angular eigenvalues respectively.

In this paper, we choose Planck units $\hbar=c=G=1$. 


\section{Kerr-de Sitter black holes}

The Kerr-de Sitter metric [29] can be written in Chambers-Moss coordinates [9] as

$$
d s^{2}=-\frac{\Delta_{r}}{\rho^{2} \chi^{4}}\left(d t-a \sin ^{2} \theta d \phi\right)^{2}+\frac{\Delta_{\theta} \sin ^{2} \theta}{\rho^{2} \chi^{4}}\left(a d t-\left(r^{2}+a^{2}\right) d \phi\right)^{2}+\rho^{2}\left(\frac{d \theta^{2}}{\Delta_{\theta}}+\frac{d r^{2}}{\Delta_{r}}\right),
$$

where

$$
\begin{array}{rlrl}
\chi^{2} & \equiv 1+\alpha^{2}, & \rho^{2} \equiv r^{2}+a^{2} \cos ^{2} \theta, \\
\Delta_{\theta} \equiv 1+\alpha^{2} \cos ^{2} \theta, & \Delta_{r} \equiv\left(r^{2}+a^{2}\right)\left(1-\frac{r^{2}}{L^{2}}\right)-2 M r
\end{array}
$$

and $\alpha \equiv a / L$. The coordinate ranges are $t, r \in \mathbb{R}, \theta \in[0, \pi], \phi \in[0,2 \pi)$. The solutions are labeled by the parameters $(a, M, L)$, where $M$ is the mass, $J=M a$ is the angular momentum and $L \equiv \sqrt{3 / \Lambda}$ is the de Sitter radius, where $\Lambda$ is the cosmological constant. In particular, the Ricci scalar is equal to $R=12 / L^{2}$. When $L \rightarrow \infty$, we recover the Kerr metric in Boyer-Lindquist coordinates and when $a=0$ we recover the Schwarzschild-de Sitter metric.

The polynomial $\Delta_{r}$ has four roots: $r_{i}, i \in \mathcal{I}$, where $\mathcal{I}=\{C,-,+,--\}$, with $r_{--}=$ $-\left(r_{-}+r_{+}+r_{C}\right)$. It is easy to check that, in order to have a black hole in a de Sitter Universe, i.e, for the two largest roots to be positive, $0<r_{+} \leq r_{C}$, then the other two roots must be real and must satisfy $r_{--}<0 \leq r_{-} \leq r_{+}<r_{C}$, and thus $r_{-}$is the inner Cauchy horizon. This implies that $r_{C}, r_{+}$and $r_{-}$are the radii of, respectively, the cosmological horizon, the (outer) event horizon and the (inner) Cauchy horizon. The negative horizon $r_{--}$is reachable if one goes inside the inner horizon and, avoiding the ring-like singularity (located at $r=0, \theta=\pi / 2$ ), goes to negative values of $r$, thus reaching another asymptotically de Sitter region. The global extension of the Kerr-de Sitter black hole is described in [9, 29-31] and is shown in figure 1.

The limit $r_{+} \rightarrow r_{-}$corresponds to an extremal black hole $\left(r_{+}=r_{-}\right.$is an event horizon), whereas $r_{+} \rightarrow r_{C}$ corresponds to an extreme naked singularity $\left(r_{+}=r_{C}\right.$ is a cosmological horizon) [31] and it is called the rotating Nariai limit [20,32]. ${ }^{2}$ The extremal black hole is a maximally-rotating black hole, and it is interesting to note that it can have $a>M$ ( $a=M$ being the maximal rotating limit in Kerr).

Two or more roots of $\Delta_{r}$ coincide if and only if the discriminant $\mathcal{D}=L^{-12} \prod_{i \neq j \in \mathcal{I}}\left(r_{i}-r_{j}\right)$ is zero. It is possible to write the discriminant in terms of only $M / L$ and $a / L$. One can show that $\mathcal{D} \geq 0$ and $M, a \geq 0$ if and only if all the roots of $\Delta_{r}$ are real and $r_{C} \geq r_{+} \geq$ $r_{-} \geq 0>r_{--}$. This condition thus defines the region in green in figure 2, corresponding to the parameter space where black hole solutions exist [9].

The QNM expansion that we calculate later in this paper is most easily organized as a series in $a$ and in the extremality parameter $\epsilon \equiv\left(r_{C}-r_{+}\right) / L$, instead of $a$ and $M$. The

\footnotetext{
${ }^{2}$ We note, however, that ref. [31] mentions that "there is no clear, universally-agreed-upon definition for naked singularities when the cosmological constant is non-zero". An observer outside the cosmological horizon $r_{C}$ would call the extremal $r_{+}=r_{C}$ solution an extremal black hole, for instance.
} 


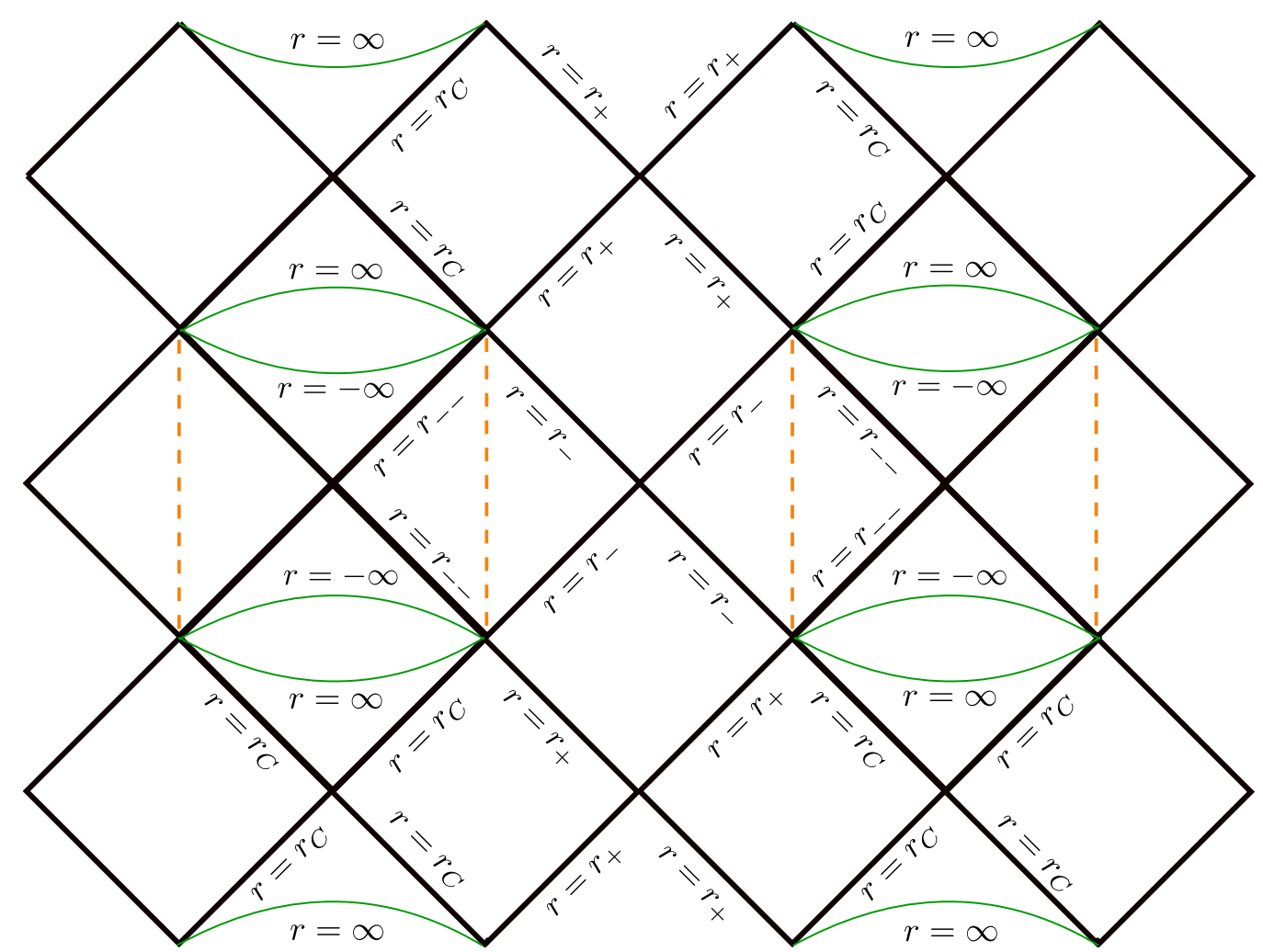

Figure 1. Kerr-de Sitter causal diagram for $\theta=0$. The ring-like singularity at $r=0, \theta=\pi / 2$ is denoted by the orange dashed lines.

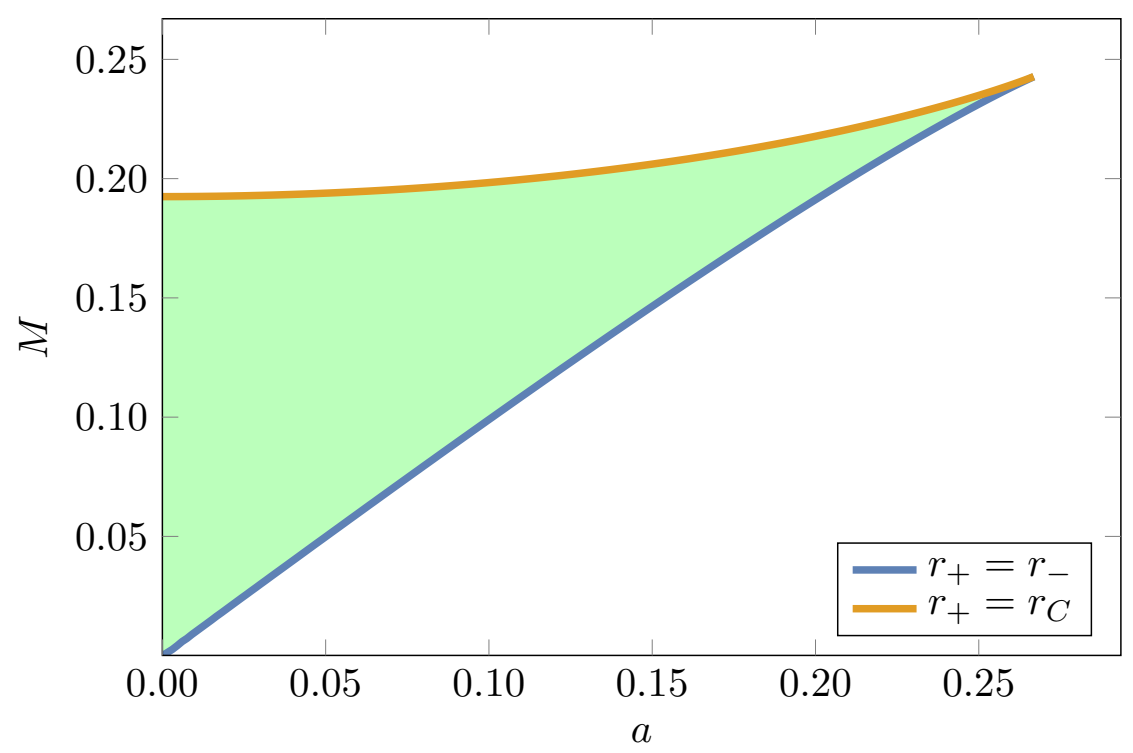

Figure 2. The green region correspond to values of $(M, a)$ (with $L=1$ ) representing black hole solutions. The blue line corresponds to $r_{+}=r_{-}$and the orange line to $r_{+}=r_{C}$. 


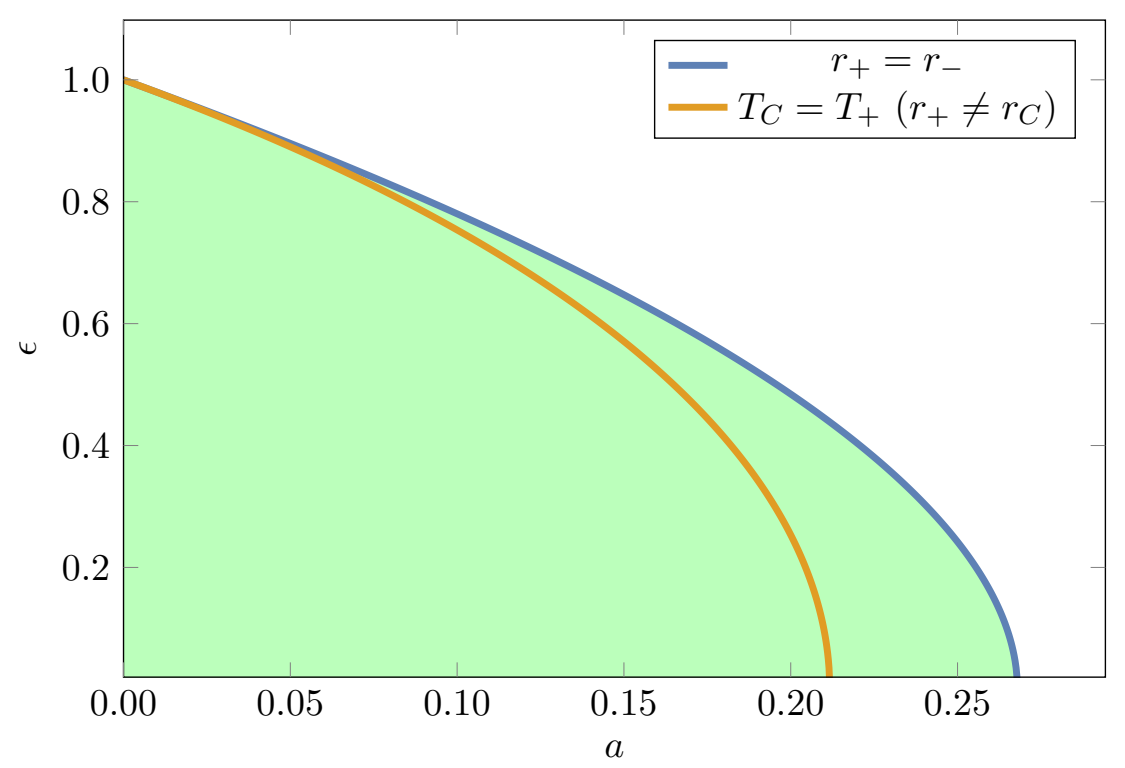

Figure 3. Kerr-de Sitter configuration space in terms of $(\epsilon, a)$ with $L=1$. The green region corresponds to the same one in figure 2 of Kerr-de Sitter black holes. The $\epsilon=0$ line corresponds to the rotating Nariai limit $r_{+}=r_{C}$ and the point $\epsilon=1, a=0$ corresponds to empty de Sitter spacetime. The blue line corresponds to the extremal limit $r_{+}=r_{-}$, while the orange line corresponds to the equal temperature condition $T_{C}=T_{+}$for $r_{+} \neq r_{C}$ (lukewarm solutions).

horizons are given by

$$
\begin{array}{r}
r_{+}=r_{C}-\epsilon L, \quad r_{--}=-r_{+}-r_{C}-r_{-}, \\
r_{C}=\frac{\epsilon L}{2}+\frac{L}{2 \sqrt{3}} \sqrt{2 \sqrt{\left(\alpha^{2}+\epsilon^{2}-1\right)^{2}-12 \alpha^{2}}-2 \alpha^{2}+\epsilon^{2}+2} \\
r_{-}=\frac{L}{2 \sqrt{3}}\left(\sqrt{-4 \sqrt{\left(\alpha^{2}+\epsilon^{2}-1\right)^{2}-12 \alpha^{2}}-8 \alpha^{2}-5 \epsilon^{2}+8}\right. \\
\left.\quad-\sqrt{2 \sqrt{\left(\alpha^{2}+\epsilon^{2}-1\right)^{2}-12 \alpha^{2}}-2 \alpha^{2}+\epsilon^{2}+2}\right) .
\end{array}
$$

Each horizon $r_{k}, k \in \mathcal{I}$, has an associated temperature $T_{k}$ and an angular velocity $\Omega_{k}$ given by

$$
T_{k}=\frac{\left|\Delta_{r}^{\prime}\left(r_{k}\right)\right|}{4 \pi \chi^{2}\left(r_{k}^{2}+a^{2}\right)}, \quad \Omega_{k}=\frac{a}{r_{k}^{2}+a^{2}},
$$

where the prime denotes derivative with respect to the argument of $\Delta_{r}(r)$. The configuration space in terms of $(\epsilon, a)$ is given in figure 3. The extremal limit corresponds to the blue line, while the rotating Nariai limit is clearly the $\epsilon=0$ line. Given the temperatures of the event and cosmological horizons, respectively, $T_{+}$and $T_{C}$, the orange line in figure 3 corresponds to the condition $T_{+}=T_{C}$ for $r_{+} \neq r_{C}$, called lukewarm solutions in [20]. As noted in [20], these solutions are not in thermal equilibrium, as the two horizons can still exchange angular momentum while keeping the temperature fixed. Finally, it is worth mentioning that, recently, it was shown that the region between the event and cosmological horizons of Kerr-de Sitter is non-linearly stable for small angular momentum [33]. 


\section{Scattering on Kerr-de Sitter black holes}

\subsection{Linear field perturbations}

The analysis of linear field perturbations is important in order to address, for example, the linear stability of the spacetime and wave scattering around black holes. The standard formalism describing spin-field perturbations of the Kerr black hole was set up by Teukolsky [6]. Teukolsky used the so-called Newman-Penrose formalism, whereby the various field components are projected onto a null tetrad. Teukolsky decoupled the equations for the spin-field perturbations in Kerr, obtaining one single master equation which is obeyed by all possible spins of the field. Furthermore, in Boyer-Lindquist coordinates, the Teukolsky master equation essentially separates into two ODEs, one for the radial coordinate and the other one for the polar angular coordinate. This approach was later generalized to a Kerr-de Sitter background spacetime in $[8,9,11]$. The two ODEs are actually of Heun type [12] for any Petrov type D spacetime with a cosmological constant [10].

We note that there are different tetrad normalization choices in the literature, but the resulting equations are easily obtained by simple transformations that do not change the linear analysis. Here we follow the conventions of [11, 21], which are different from those in $[7,9]$. We next review the radial and angular equations for linear spin-field perturbations of Kerr-de Sitter.

Let $\psi_{s, \omega, m}(t, \phi, r, \theta)=e^{-i \omega t} e^{i m \phi} R_{s, \omega, m}(r) S_{s, \omega, m}(\theta)$ be a mode solution of the master equation for the Kerr-de Sitter metric in Chambers-Moss coordinates (2.1). ${ }^{3}$ Here, $\omega \in \mathbb{C}$ is the mode frequency and $m \in \mathbb{Z}$ its azimuthal number. The spin-s master equation separates into the following angular and radial ODEs $[8,9,11]$ :

$$
\begin{array}{r}
{\left[\partial_{u} \Delta_{u} \partial_{u}-\frac{1}{\Delta_{u}}\left(H+\frac{s}{2} \Delta_{u}^{\prime}\right)^{2}+2 s H^{\prime}-X_{s}\right] S_{s, \omega, m}(u)=0,} \\
{\left[\Delta_{r}^{-s} \partial_{r} \Delta_{r}^{s+1} \partial_{r}+\frac{1}{\Delta_{r}}\left(W^{2}-i s W \Delta_{r}^{\prime}\right)+2 i s W^{\prime}-Y_{s}\right] R_{s, \omega, m}(r)=0,}
\end{array}
$$

where

$$
W(r) \equiv \chi^{2}\left[\omega\left(r^{2}+a^{2}\right)-a m\right], \quad H(u) \equiv \chi^{2}\left[a \omega\left(1-u^{2}\right)-m\right]
$$

and

$$
\begin{array}{rlrl}
Y_{s}(r) & \equiv \frac{2}{L^{2}}(s+1)(2 s+1) r^{2}+{ }_{s} \lambda_{\ell m}-s\left(1-\alpha^{2}\right), & X_{s}(u) & \equiv 2\left(2 s^{2}+1\right) \alpha^{2} u^{2}-{ }_{s} \lambda_{\ell m}, \\
u & \equiv \cos \theta, & \Delta_{u}(u) \equiv\left(1-u^{2}\right)\left(1+\alpha^{2} u^{2}\right),
\end{array}
$$

with ${ }_{s} \lambda_{\ell m}={ }_{s} \lambda_{\ell m}(a \omega, \alpha)$ being the angular eigenvalue. ${ }^{4}$ The prime in $\Delta_{r}^{\prime}$ denotes derivative with respect to $r$ and in $\Delta_{u}^{\prime}$ with respect to $u$. Notice that the $s=0$ radial equation above

\footnotetext{
${ }^{3}$ The mode solutions, as well as various other quantities throughout the paper, depend on the value of the angular eigenvalue, which is usually labeled by the multipolar index $\ell$. In this paper, we omit this label unless strictly necessary, in order to avoid index cluttering.

${ }^{4}$ Our angular eigenvalue ${ }_{s} \lambda_{\ell m}$, when evaluated in Kerr (i.e, for $L \rightarrow \infty$ ), is equal to $\lambda+s$, where $\lambda$ is in Teukolsky's [6].
} 
corresponds to the Klein-Gordon equation with conformal coupling [21], i.e., with Ricci scalar coupling parameter $\xi=1 / 6$.

The solutions of the angular equation (3.1a), assuming regularity at $u=-1$ and +1 , are usually called (Anti-)de Sitter spheroidal harmonics [7] and its eigenvalues ${ }_{s} \lambda_{\ell m}=$ ${ }_{s} \lambda_{\ell m}(a \omega, \alpha)$ are only known numerically [21] or as an expansion to order $\mathcal{O}\left(\alpha^{2},(a \omega)^{2}\right)$, as derived in [11].

The radial and angular ODEs have certain symmetries. In particular, eq. (3.1a) is symmetric under $(s, \omega, m) \rightarrow(-s,-\omega,-m)$. This implies that ${ }_{-s} \lambda_{\ell,-m}(a \omega, \alpha)={ }_{s} \lambda_{\ell m}(-a \omega, \alpha)$ and a similar symmetry for the angular eigenfunction. Moreover, one can also show that $\Delta_{r}^{-s} R_{-s,-\omega,-m}$ is a solution of (3.1b) if $R_{s, \omega, m}$ is a solution.

\subsection{Reduction of radial and angular equations to Heun equations}

In the following, we want to analyze the connection problem for the angular and radial equations (3.1). By the connection problem we mean rewriting the solution near a certain singular point of the differential equation in terms of a linear combination of solutions near another singular point. The tasks of finding the eigenvalues of the angular equation and the QNMs of the radial equation can both be described in terms of the connection problem $[28,34,35]$, as we show in the next subsection. It is thus helpful to reduce the radial and angular equations to some known special form, so that we can understand the structure of the solutions. In this section, we are going to apply Möbius transformations to the radial and angular variables $r$ and $u$. This will map the roots of the polynomials $\Delta_{r}(r)$ and $\Delta_{u}(u)$ to $\left(z_{k}\right)_{k \in \mathcal{J}} \equiv(0,1, x, \infty)$, with $\mathcal{J} \equiv\{0,1, x, \infty\}$.

Equations (3.1a) and (3.1b) naively possess five singular points each. In the radial case, these points are the four roots of $\Delta_{r}(r)$, i.e., $r_{k}, k \in \mathcal{I}=\{C,-,+,--\}$, together with the point $r=\infty$. In the angular case, the singular points are the four roots of $\Delta_{u}(u)$, i.e. $u= \pm 1, \pm \frac{i}{\alpha}$, together with the point $u=\infty$. For $\Lambda \neq 0$, all singular points of the radial and angular equations are regular and thus both equations are Fuchsian. Surprisingly, as firstly shown in [11], both equations can be reduced to Heun equations [12], i.e., Fuchsian equations with four regular singular points. After a series of transformations, the points $r=\infty$ and $u=\infty$ cease to be singular points of the corresponding ODEs, thus being characterized as removable singularities [36]. We rederive this result in a systematic way in appendix A. On the other hand, for $\Lambda=0$ there is a confluence of singular points, producing irregular singular points at $r=\infty$ and at $u=\infty$. Both radial and angular equations then become confluent Heun equations [34, 37]. In the following, we derive the Heun equations from the angular (3.1a) and radial (3.1b) equations.

Generic Heun equation. The basis of our method is to classify the Heun equation in terms of its monodromy data. For a function $y=y(z)$, the Heun equation can be written in the canonical form (see appendix A or $[12,36]$ )

$$
\frac{d^{2} y}{d z^{2}}+\left(\frac{1-2 \theta_{0}}{z}+\frac{1-2 \theta_{1}}{z-1}+\frac{1-2 \theta_{x}}{z-x}\right) \frac{d y}{d z}+\left(\frac{b_{1} b_{2}}{z(z-1)}-\frac{x(x-1) K_{x}}{z(z-1)(z-x)}\right) y(z)=0,
$$

where the singular points are $\left(z_{k}\right)_{k \in \mathcal{J}} \equiv(0,1, x, \infty)$, with $x \in \mathbb{C}$. The coefficients $\boldsymbol{\theta} \equiv\left(\theta_{0}, \theta_{1}, \theta_{x}, \theta_{\infty}\right)$, also known as the local monodromy coefficients (to which we shall 
simply refer as monodromies), correspond to half of the difference of Frobenius exponents of Frobenius series solutions $y_{k}^{(j)}, j=1,2$ close to the singular points. That is,

$$
y_{k}^{(1)}(z)=\left(z-z_{k}\right)^{2 \theta_{k}}\left(1+\mathcal{O}\left(z-z_{k}\right)\right), \quad y_{k}^{(2)}(z)=\left(1+\mathcal{O}\left(z-z_{k}\right)\right), \quad k=0,1, x .
$$

In their turn, $b_{1}$ and $b_{2}$ are the Frobenius exponents at $z=\infty$ :

$$
y_{\infty}^{(1)}(z)=z^{-b_{1}}\left(1+\mathcal{O}\left(z^{-1}\right)\right), \quad y_{\infty}^{(2)}(z)=z^{-b_{2}}\left(1+\mathcal{O}\left(z^{-1}\right)\right),
$$

with

$$
2 \theta_{\infty} \equiv b_{1}-b_{2} .
$$

The regularity of $z=\infty$ in (3.5) implies the Fuchs condition

$$
b_{1}+b_{2}+\sum_{k \in \mathcal{J}} 2 \theta_{k}=2 .
$$

The two relations in eqs. (3.8) and (3.9) can be used to write the Frobenius exponents $b_{1,2}$ in terms of the $\theta$ 's as

$$
b_{1}=-\sum_{k \in \mathcal{J}} \theta_{k}+1+\theta_{\infty}, \quad b_{2}=-\sum_{k \in \mathcal{J}} \theta_{k}+1-\theta_{\infty} .
$$

The accessory parameter $K_{x}$ is the only parameter which, for generic monodromy data, cannot be written only in terms of $\theta$ 's, as it also depends on global information of the solutions. Therefore, the Heun equation is completely determined by five parameters for fixed moduli parameter $x$ : $\mathcal{M}_{\text {Heun }} \equiv\left(\theta_{0}, \theta_{1}, \theta_{x}, \theta_{\infty} ; K_{x}\right)$.

Angular equation. The Frobenius exponents of local solutions of (3.1a) around $\left(u_{k}\right)_{k \in \mathcal{J}} \equiv\left(-1, \frac{i}{\alpha}, 1,-\frac{i}{\alpha}\right)$ are given by

$$
\theta_{k}=-\operatorname{res}_{u=u_{k}} \frac{H(u)}{\Delta_{u}(u)}-\frac{s}{2}, k \in \mathcal{J} .
$$

Explicitly, the difference of Frobenius exponents is given by

$$
\begin{aligned}
& 2 \theta_{0}=m-s, \quad 2 \theta_{1}=\frac{i\left(\alpha^{2}+1\right) a \omega}{\alpha}-i \alpha m-s, \\
& 2 \theta_{x}=-m-s, \quad 2 \theta_{\infty}=-\frac{i\left(\alpha^{2}+1\right) a \omega}{\alpha}+i \alpha m-s .
\end{aligned}
$$

Starting with eq. (3.1a), we make the coordinate transformation

$$
z(u)=\zeta_{\infty} \frac{u+1}{u+i / \alpha}, \quad \zeta_{\infty}=\frac{2 i}{(i+\alpha)},
$$

whereby

$$
\left(u_{k}\right)_{k \in \mathcal{J}}=\left(-1, \frac{i}{\alpha}, 1,-\frac{i}{\alpha}\right) \mapsto\left(z_{k}\right)_{k \in \mathcal{J}}=(0,1, x, \infty)
$$

and

$$
x=\frac{4 i \alpha}{(i+\alpha)^{2}} .
$$


We also make the s-homotopic transformation [36]

$$
S_{s, \omega, m}(u)=z^{-\theta_{0}}(z-1)^{-\theta_{1}}(z-x)^{-\theta_{x}}\left(z-\zeta_{\infty}\right) f(z),
$$

which, as proven in appendix A.2, reduces (3.1a) to the Heun equation

$$
\begin{aligned}
& \frac{d^{2} f}{d z^{2}}+\left(\frac{1-2 \theta_{0}}{z}+\frac{1-2 \theta_{1}}{z-1}+\frac{1-2 \theta_{x}}{z-x}\right) \frac{d f}{d z}+ \\
& \quad+\left(\frac{(1+2 s)\left(1+2 s+2 \theta_{\infty}\right)}{z(z-1)}-\frac{x(x-1) K_{x}}{z(z-1)(z-x)}\right) f=0
\end{aligned}
$$

The accessory parameter is

$$
\begin{aligned}
K_{x}= & \frac{(2 s+1)\left(\theta_{0}+\theta_{x}+s\right)-s}{x}+\frac{(2 s+1)\left(\theta_{1}+\theta_{x}+s\right)-s}{x-1} \\
& +\frac{2 s^{2}+1}{\zeta_{\infty}-x}-\frac{1}{4 \zeta_{\infty}^{2}} \frac{2\left(2 s^{2}+1\right) x^{2}+{ }_{s} \lambda \lambda_{\ell m} \zeta_{\infty}^{4}}{x(x-1)}
\end{aligned}
$$

where we used the fact that $\alpha^{2}=-x^{2} / \zeta_{\infty}^{4}$. Notice that

$$
\sum_{k \in \mathcal{J}} \theta_{k}=-2 s-\sum_{k \in \mathcal{J}} H_{k}=-2 s
$$

as the sum of residues $\sum_{k} H_{k}=\sum_{k} \operatorname{res}_{u=u_{k}}\left(H(u) / \Delta_{u}(u)\right)$ is zero from the Cauchy theorem. It should be clear that the extra property (3.19) is not generally true for an arbitrary Heun equation; it is a special condition for the black hole case and reduces the number of parameters from five to four, as one of the monodromies is determined in terms of the others.

The $x \rightarrow 0(\alpha=a / L \rightarrow 0)$ limit of (3.17) is regular for $L$ fixed, as neither the $\theta_{k}$ 's nor " $x(x-1) K_{x}$ " diverge, whereas the coefficients diverge when taking the same limit but with $a$ fixed, instead of $L$. The first limit is the Schwarzschild-de Sitter limit, whereas the second one corresponds to the Kerr limit. In the limit $a \rightarrow 0$, we have ${ }_{s} \lambda_{\ell m} \rightarrow \ell(\ell+1)-s^{2}$.

Radial equation. The Frobenius exponents of the radial solutions are $\theta_{k}=i W_{k}+s / 2$, where $W_{k} \equiv \operatorname{res}_{r=r_{k}} \frac{W(r)}{\Delta_{r}(r)}, k \in \mathcal{J}$, are residues (see appendix A.1). Explicitly,

$$
\theta_{k}=i \chi^{2}\left(\frac{\omega\left(r_{k}^{2}+a^{2}\right)-a m}{\Delta_{r}^{\prime}\left(r_{k}\right)}\right)+\frac{s}{2}= \pm \frac{i}{4 \pi}\left(\frac{\omega-\Omega_{k} m}{T_{k}}\right)+\frac{s}{2}, \quad k \in \mathcal{J} .
$$

The plus or minus sign in (3.20) arises from the sign of $\Delta_{r}^{\prime}\left(r_{k}\right)$, chosen in such a way that the temperatures $T_{k}$ are positive $[9,38]$. From the residue theorem, we have that

$$
\sum_{k \in \mathcal{J}} \theta_{k}=2 s
$$

which implies, from eq. (3.10), that $b_{2}=1-2 s$ and $b_{1}=1-2 s+\theta_{\infty}$. In order to obtain the Heun equation from eq. (3.1b), we apply the coordinate transformation

$$
z(r)=\zeta_{\infty} \frac{r-r_{C}}{r-r_{--}}, \quad \zeta_{\infty}=\frac{r_{-}-r_{--}}{r_{-}-r_{C}}, \quad x=\zeta_{\infty} \frac{r_{+}-r_{C}}{r_{+}-r_{--}},
$$


corresponding to the mapping

$$
r=\left(r_{--}, r_{-}, r_{+}, r_{C}, \infty\right) \quad \mapsto \quad z=\left(\infty, 1, x, 0, \zeta_{\infty}\right), \quad 0<x<1,
$$

and the s-homotopic transformation

$$
f(z)=z^{\frac{s}{2}+\theta_{0}}(z-1)^{\frac{s}{2}+\theta_{1}}(z-x)^{\frac{s}{2}+\theta_{x}}\left(z-\zeta_{\infty}\right)^{-1-2 s} R_{s, \omega, m}(r) .
$$

The transformations (3.22) and (3.24) result in the Heun equation

$$
\begin{aligned}
& \frac{d^{2} f}{d z^{2}}+\left(\frac{1-2 \theta_{0}}{z}+\frac{1-2 \theta_{1}}{z-1}+\frac{1-2 \theta_{x}}{z-x}\right) \frac{d f}{d z}+ \\
& \quad+\left(\frac{(1-2 s)\left(1-2 s+2 \theta_{\infty}\right)}{z(z-1)}-\frac{x(x-1) K_{x}}{z(z-1)(z-x)}\right) f=0
\end{aligned}
$$

According to (A.40), the accessory parameter in (3.25) is given by

$$
\begin{aligned}
K_{x}= & \frac{(1-2 s)\left(\theta_{x}+\theta_{0}-s\right)+2 s}{x}+\frac{(1-2 s)\left(\theta_{x}+\theta_{1}-s\right)+2 s}{x-1}- \\
& -\frac{(1+s)(1+2 s)}{x-\zeta_{\infty}}+\frac{L^{2}}{x(x-1)} \frac{2(2 s+1)(s+1) r_{+}^{2} / L^{2}+{ }_{s} \lambda_{\ell m}-s\left(1-a^{2} / L^{2}\right)}{\left(r_{C}-r_{-}\right)\left(r_{+}-r_{--}\right)} .
\end{aligned}
$$

Notice that we use some of the same symbols in the radial and in the angular equations, but it should be clear from the context whether such symbol corresponds to the radial or to the angular case.

The limits $x \rightarrow 0$ and $x \rightarrow 1$ correspond to the two possible extremal limits: $r_{+} \rightarrow r_{C}$ and $r_{+} \rightarrow r_{-}$, respectively. If we do not make any assumptions on $\omega$, two of the $\theta_{k}$ 's diverge in each of these limits and so the resulting confluence yields an irregular singular point (confluent Heun equation). However, if we expand $\omega=m \Omega_{+}+x \tilde{\omega}+\mathcal{O}\left(x^{2}\right)$ (or starting the series with $m \Omega_{C}$ ), with some coefficient $\tilde{\omega}$, and take the limit $x \rightarrow 0$ in eq. (3.25), then the $\theta_{k}$ 's are all finite and we obtain a regular singularity from the coalescence of singularities. We can also expand $\omega=m \Omega_{+}+(1-x) \tilde{\omega}+\mathcal{O}\left((x-1)^{2}\right)$ (or starting with $m \Omega_{-}$), take the limit $x \rightarrow 1$ to reach the same conclusion. ${ }^{5}$ As we show below, this procedure for $x \rightarrow 0$ gives the near-horizon, near-extremal limit associated to the rotating Nariai limit.

\subsection{Kerr-de Sitter connection coefficients and greybody factor}

A standard approach for analytically calculating (approximations to) scattering coefficients is to match series expansions near each singular point of the radial ODE in an intermediate region. In many cases, the radial ODE can be solved exactly in the asymptotic regions in terms of known special functions (such as hypergeometric functions) - see, e.g., [39-41] for QNMs in near-extremal Kerr. However, when trying that approach for the Kerr-(Anti-)de Sitter family of solutions, one obtains a Heun or confluent Heun equation in the simplest of the cases. Therefore, in order to reduce the ODEs in these cases to the hypergeometric

\footnotetext{
${ }^{5}$ These observations are compatible with the definition of confluence in [36]: a coalescence of singularities only increases the rank of the resulting singularity if some of the parameters of the equation diverge as $x \rightarrow 0$ (or, similarly, as $x \rightarrow 1$ or $x \rightarrow \infty$ ).
} 
equation, one needs to make some extra assumption, such as low frequency [42] or large (Anti)-de Sitter radius [43].

Another approach for calculating scattering coefficients in the case of Heun equations is the so-called Mano-Suzuki-Takasugi (MST) method [12, 44-46]. This method consists of expressing the solutions as infinite series of hypergeometric functions, and it was used in $[11,47,48]$ in Kerr-de Sitter. Given a certain augmented convergence condition, these series solutions converge in an ellipsis with two singular points as foci.

Here we take another path and use the monodromy approach to scattering amplitudes, first discussed in [34] and later generalized in [28, 35, 37]. It is worth mentioning that a monodromy approach for calculating QNMs with large imaginary part has been developed in, e.g., $[49,50]$.

Ingoing and outgoing solutions. Let us first focus on the radial equation (3.1b) supposing that we know the angular eigenvalue ${ }_{s} \lambda_{\ell m}$. For definiteness, we consider the problem of connecting a local solution near the black hole horizon $r_{+}$to a local solution near the cosmological horizon $r_{C}$. Let us define two linearly independent solutions $R_{ \pm}^{(j)}(r)$ by their local behaviour near $r=r_{j}$, for $j=+, C$, as

$$
\begin{array}{ll}
R_{ \pm}^{(+)}(r)=\left(r-r_{+}\right)^{-\frac{s}{2} \pm \theta_{+}}\left(1+\mathcal{O}\left(r-r_{+}\right)\right), & \theta_{+}=i \frac{\tilde{\omega}_{+}}{2 \kappa_{+}}+\frac{s}{2} \\
R_{ \pm}^{(C)}(r)=\left(r-r_{C}\right)^{-\frac{s}{2} \pm \theta_{C}}\left(1+\mathcal{O}\left(r-r_{C}\right)\right), & \theta_{C}=-i \frac{\tilde{\omega}_{C}}{2 \kappa_{C}}+\frac{s}{2},
\end{array}
$$

where

$$
\tilde{\omega}_{j} \equiv \omega-m \Omega_{j}, \quad \kappa_{j} \equiv 2 \pi T_{j}
$$

and $\kappa_{j}$ is the surface gravity (see eq. (2.4) for the angular velocity and temperature definitions and eq. (3.20) for the $\theta$ 's).

The notion of ingoing and outgoing solutions is essential to define a scattering problem. Here these notions should match the ingoing and outgoing null coordinates in Kerr-de Sitter, $(v, u)$ respectively:

$$
d t=d v-\frac{\chi^{2}\left(r^{2}+a^{2}\right)}{\Delta_{r}} d r, \quad d t=d u+\frac{\chi^{2}\left(r^{2}+a^{2}\right)}{\Delta_{r}} d r,
$$

which in turn motivate the definition of the radial tortoise coordinate $r_{*}$ as

$$
d r_{*}=\frac{\chi^{2}\left(r^{2}+a^{2}\right)}{\Delta_{r}} d r
$$

This implies that $r_{*} \rightarrow+\infty$ as $r \rightarrow r_{C}$ and $r_{*} \rightarrow-\infty$ as $r \rightarrow r_{+}$. Partly in terms of $r_{*}$, the above local solutions may be written as

$$
R_{ \pm}^{(j)}(r) \propto\left(r-r_{j}\right)^{-\frac{1}{2}(s \mp s)} e^{ \pm i \tilde{\omega}_{j} r_{*}}\left(1+\mathcal{O}\left(r-r_{j}\right)\right), \quad j=C,+.
$$

Therefore, travelling waves have the form $\exp \left(-i\left(\omega t \pm \tilde{\omega}_{j} r_{*}\right)\right)$ and the phase velocity depends on the relative sign of the real part of the frequencies, i.e.,

$$
\begin{array}{lll}
\Re(\omega) \Re\left(\tilde{\omega}_{j}\right)>0 & \Rightarrow & R_{+}^{(j)} \text { is outgoing and } R_{-}^{(j)} \text { is ingoing at } r=r_{j}, \\
\Re(\omega) \Re\left(\tilde{\omega}_{j}\right)<0 & \Rightarrow & R_{+}^{(j)} \text { is ingoing and } R_{-}^{(j)} \text { is outgoing at } r=r_{j} .
\end{array}
$$


In our case, as $r_{C} \geq r_{+}>0$, we have that $\Omega_{+} \geq \Omega_{C}$. This allows for the possibility $|m| \Omega_{+}>|\Re(\omega)|>|m| \Omega_{C}$, which for $\omega \in \mathbb{R}$ corresponds to the superradiant regime, valid only for bosons $[48,51]$. The physical definition of superradiance is that the net flux of radiation with respect to each horizon should have alternate signs. Given that the notion of ingoing and outgoing is equivalent to the sign of the radiation flux, for $\omega \in \mathbb{R}$, we have

$$
\begin{array}{lll}
\tilde{\omega}_{C} \tilde{\omega}_{+} \geq 0 & \Rightarrow \quad \text { no superradiance } \\
\tilde{\omega}_{C} \tilde{\omega}_{+}<0 \quad \Rightarrow \quad \text { superradiance. }
\end{array}
$$

For further details, we refer the reader to [48]. Notice that definitions (3.32) and (3.33) are invariant under $(\omega, m) \rightarrow(-\omega,-m)$.

Connection coefficients for radial solutions. We now wish to obtain formulas relating the local solutions $R_{ \pm}^{(j)}, j=+, C$. This means writing a set of local solutions at a singular point as a linear combination of a set of local solutions at another singular point in terms of certain connection coefficients: transmission coefficients $\mathcal{T}_{s}$ and reflection coefficients $\mathcal{R}_{s}$. These coefficients become scattering coefficients if the frequency is real, in which case we can consistently define the radiation flux at each one of the horizons. We classify the scattering problem between $r_{+}$and $r_{C}$ into two possibilities with respect to the boundary conditions: purely ingoing or purely outgoing solutions at one of the horizons.

We define the $I N$ mode by imposing the boundary conditions [40],

$$
R_{\mathrm{in} ; s}^{(+)} \sim\left\{\begin{array}{lr}
\frac{1}{\mathcal{T}_{s}} R_{-}^{(C)}-\frac{\mathcal{R}_{s}}{\mathcal{T}_{s}} R_{+}^{(C)}, & r \rightarrow r_{C}, \\
R_{-}^{(+)}, & r \rightarrow r_{+},
\end{array}\right.
$$

where $\mathcal{T}_{s}=\mathcal{T}_{s}(\omega, m)$ and $\mathcal{R}_{s}=\mathcal{R}_{s}(\omega, m)$ are complex connection coefficients. If $\Re(\omega) \Re\left(\tilde{\omega}_{j}\right)>0$, this corresponds to a purely ingoing solution at $r_{+}$. We can obtain another linearly independent solution from (3.34) via the discrete transformation

$$
\star: f_{s}(\omega, m) \mapsto f_{s}^{\star}(\omega, m) \equiv f_{-s}(-\omega,-m),
$$

and multiplying the solution by $\Delta_{r}^{-s}$, i.e.,

$$
R_{\text {out } ; s}^{(+)} \equiv \Delta_{r}^{-s}\left(R_{\text {in;s }}^{(+)}\right)^{\star} \sim \begin{cases}\left(\Delta_{r}^{\prime}\left(r_{C}\right)\right)^{-s}\left(\frac{1}{\mathcal{T}_{s}^{\star}} R_{+}^{(C)}-\frac{\mathcal{R}_{s}^{\star}}{\mathcal{T}_{s}^{\star}} R_{-}^{(C)}\right), & r \rightarrow r_{C}, \\ \left(\Delta_{r}^{\prime}\left(r_{+}\right)\right)^{-s} R_{+}^{(+)}, & r \rightarrow r_{+} .\end{cases}
$$

If $\omega \in \mathbb{R}$, then $(\omega, m) \rightarrow(-\omega,-m)$ is equivalent to taking the complex conjugate solution, and so

$$
R_{\text {out } ; s}^{(+)}=\Delta_{r}^{-s}\left(R_{\mathrm{in} ;-s}^{(+)}\right)^{*} .
$$

This implies that $\mathcal{R}_{s}^{\star}=\mathcal{R}_{-s}^{*}$ and $\mathcal{T}_{s}^{\star}=\mathcal{T}_{-s}^{*}$ when $\omega \in \mathbb{R}$.

The "Wronskian" between two solutions is

$$
\mathcal{W}_{s}\left[u_{1} ; u_{2}\right] \equiv-i \Delta_{r}^{s+1}(r)\left(u_{1} \frac{d u_{2}}{d r}-u_{2} \frac{d u_{1}}{d r}\right) \text {. }
$$


It is a simple fact that this current is constant in $r$, as long as $u_{1}$ and $u_{2}$ obey the same radial equation (3.1b). Evaluating the quantities at $r=r_{+}$, we obtain the following expression:

$$
\left.\mathcal{W}_{\text {hor }}^{(+)} \equiv \mathcal{W}_{s}\left[R_{\text {in;s }}^{(+)} ; R_{\text {out; } s}^{(+)}\right]\right|_{r=r_{+}}=-2 i \theta_{+} \Delta_{r}^{\prime}\left(r_{+}\right),
$$

whose real part represents the flux of radiation transmitted into the black hole when $\omega \in \mathbb{R}$. Evaluating the quantities at $r=r_{C}$, yields for the total flux,

$$
\left.\mathcal{W}_{\text {cosm }}^{(+)} \equiv \mathcal{W}_{s}\left[R_{\mathrm{in} ; s}^{(+)} ; R_{\text {out } ; s}^{(+)}\right]\right|_{r=r_{C}}=2 i \theta_{C} \Delta_{r}^{\prime}\left(r_{C}\right) \frac{\left(\mathcal{R}_{s} \mathcal{R}_{s}^{\star}-1\right)}{\mathcal{T}_{s} \mathcal{T}_{s}^{\star}}=\mathcal{W}_{\text {ref }}^{(+)}+\mathcal{W}_{\mathrm{in}}^{(+)},
$$

where

$$
\mathcal{W}_{\text {in }}^{(+)} \equiv-2 i \theta_{C} \Delta_{r}^{\prime}\left(r_{C}\right) \frac{1}{\mathcal{T}_{s} \mathcal{T}_{s}^{\star}}, \quad \mathcal{W}_{\text {ref }}^{(+)} \equiv 2 i \theta_{C} \Delta_{r}^{\prime}\left(r_{C}\right) \frac{\mathcal{R}_{s} \mathcal{R}_{s}^{\star}}{\mathcal{T}_{s} \mathcal{T}_{s}^{\star}}
$$

Because the flux is conserved, $\mathcal{W}_{\text {cosm }}^{(+)}=\mathcal{W}_{\text {hor }}^{(+)}$and thus

$$
\mathcal{R}_{s} \mathcal{R}_{s}^{\star}+\tau_{s} \mathcal{T}_{s} \mathcal{T}_{s}^{\star}=1, \quad \tau_{s}(\omega, m) \equiv \frac{\theta_{+} \Delta_{r}^{\prime}\left(r_{+}\right)}{\theta_{C} \Delta_{r}^{\prime}\left(r_{C}\right)}=\frac{2 \chi^{2}\left(r_{+}^{2}+a^{2}\right) \tilde{\omega}_{+}-i s \Delta_{r}^{\prime}\left(r_{+}\right)}{2 \chi^{2}\left(r_{C}^{2}+a^{2}\right) \tilde{\omega}_{C}-i s \Delta_{r}^{\prime}\left(r_{C}\right)} .
$$

The greybody factor $G_{s}(\omega, m)$ is defined to be the ratio between the horizon flux and the incoming flux

$$
G_{s}(\omega, m)=\frac{\mathcal{W}_{\mathrm{hor}}^{(+)}}{\mathcal{W}_{\text {in }}^{(+)}}=\tau_{s}(\omega, m) \mathcal{T}_{s}(\omega, m) \mathcal{T}_{s}^{\star}(\omega, m) .
$$

For $\omega \in \mathbb{R}$ and outside the superradiant regime, the greybody factor must be real and positive. This has been proven in [48] using the Teukolsky-Starobinsky identities, which relate $\mathcal{T}_{-s}$ with $\mathcal{T}_{s}$. This procedure also makes the superradiance regime explicit. We refer the reader to [48] for details.

We can do the same exercise for the UP mode, defined via the following boundary condition at the cosmological horizon:

$$
R_{\text {out } ; s}^{(C)} \sim \begin{cases}R_{+}^{(C)}, & r \rightarrow r_{C}, \\ \frac{1}{\tilde{\mathcal{T}}_{s}} R_{+}^{(+)}-\frac{\tilde{\mathcal{R}}_{s}}{\tilde{\mathcal{T}}_{s}} R_{-}^{(+)}, & r \rightarrow r_{+},\end{cases}
$$

and the reflected mode, which is defined via the boundary condition:

$$
R_{\mathrm{in} ; s}^{(C)} \sim \begin{cases}\left(\Delta_{r}^{\prime}\left(r_{C}\right)\right)^{-s} R_{-}^{(C)}, & r \rightarrow r_{C}, \\ \left(\Delta_{r}^{\prime}\left(r_{+}\right)\right)^{-s}\left(\frac{1}{\tilde{\mathcal{T}}_{s}^{\star}} R_{-}^{(+)}-\frac{\tilde{\mathcal{R}}_{s}^{\star}}{\tilde{\mathcal{T}}_{s}^{\star}} R_{+}^{(+)}\right), & r \rightarrow r_{+},\end{cases}
$$

where $\tilde{\mathcal{T}}_{s}^{\star} \equiv \tilde{\mathcal{T}}_{-s}(-\omega,-m)$ and $\tilde{\mathcal{R}}_{s}^{\star} \equiv \tilde{\mathcal{R}}_{-s}(-\omega,-m)$. We then obtain similar results for the flux as above for the purely in/outgoing solutions on the event horizon:

$$
\begin{aligned}
& \left.\mathcal{W}_{\text {cosm }}^{(C)} \equiv \mathcal{W}_{s}\left[R_{\text {in } ; s}^{(C)} ; R_{\text {out } ; s}^{(C)}\right]\right|_{r=r_{C}}=-2 i \theta_{C} \Delta_{r}^{\prime}\left(r_{C}\right), \\
& \left.\mathcal{W}_{\text {hor }}^{(C)} \equiv \mathcal{W}_{s}\left[R_{\text {in } ; s}^{(C)} ; R_{\text {out } ; s}^{(C)}\right]\right|_{r=r_{+}}=2 i \theta_{+} \Delta_{r}^{\prime}\left(r_{+}\right) \frac{\left(\tilde{\mathcal{R}}_{s} \tilde{\mathcal{R}}_{s}^{\star}-1\right)}{\tilde{\mathcal{T}}_{s} \tilde{\mathcal{T}}_{s}^{\star}}=\mathcal{W}_{\text {ref }}^{(+)}+\mathcal{W}_{\text {in }}^{(+)} .
\end{aligned}
$$


Then, $\mathcal{W}_{\text {cosm }}^{(C)}=\mathcal{W}_{\text {hor }}^{(C)}$ implies that

$$
\tilde{\mathcal{R}}_{s} \tilde{\mathcal{R}}_{s}^{\star}+\tau_{s}^{-1} \tilde{\mathcal{T}}_{s} \tilde{\mathcal{T}}_{s}^{\star}=1
$$

Now, writing $\tau_{s}=\left|\tau_{s}\right| e^{i \phi}, \phi=\arg \left(\tau_{s}\right)$, we define the vectors

$$
\begin{aligned}
& \Phi_{+}(r) \equiv\left|\tau_{s}\right|^{-1 / 2} e^{-i \phi / 2}\left(R_{\text {in } ; s}^{(+)}(r) \quad R_{\text {out } ; s}^{(+)}(r)\right), \\
& \Phi_{C}(r) \equiv\left(R_{\text {in } ; s}^{(C)}(r) R_{\text {out } ; s}^{(C)}(r)\right)
\end{aligned}
$$

from the above linearly independent solutions. Then it is straightforward to show that $[34,40]$

$$
\Phi_{+} \mathcal{M}_{+C}=\Phi_{C}
$$

with the connection matrix given by

$$
\mathcal{M}_{+C}=\left(\begin{array}{cc}
\left(\overline{\mathcal{T}}_{s}^{\star}\right)^{-1} & \overline{\mathcal{R}}_{s}\left(\overline{\mathcal{T}}_{s}\right)^{-1} \\
\overline{\mathcal{R}}_{s}^{\star}\left(\overline{\mathcal{T}}_{s}^{\star}\right)^{-1} & \left(\overline{\mathcal{T}}_{s}\right)^{-1}
\end{array}\right)
$$

with

$$
\begin{aligned}
\overline{\mathcal{T}}_{s} \equiv\left|\tau_{s}\right|^{1 / 2} e^{i \phi / 2}\left(\Delta_{r}^{\prime}\left(r_{C}\right)\right)^{-s} \mathcal{T}_{s}, & \overline{\mathcal{R}}_{s} \equiv\left(\Delta_{r}^{\prime}\left(r_{C}\right)\right)^{-s} \mathcal{R}_{s}, \\
\overline{\mathcal{T}}_{s}^{\star} \equiv\left|\tau_{s}\right|^{1 / 2} e^{i \phi / 2}\left(\Delta_{r}^{\prime}\left(r_{C}\right)\right)^{s} \mathcal{T}_{s}^{\star}, & \overline{\mathcal{R}}_{s}^{\star} \equiv\left(\Delta_{r}^{\prime}\left(r_{C}\right)\right)^{s} \mathcal{R}_{s}^{\star} .
\end{aligned}
$$

The condition of unit determinant for $\mathcal{M}_{+C}$ is equivalent to the current conservation (3.42)

$$
\overline{\mathcal{R}}_{s} \overline{\mathcal{R}}_{s}^{\star}+\overline{\mathcal{T}}_{s} \overline{\mathcal{T}}_{s}^{\star}=1
$$

\subsection{Monodromy approach to angular eigenvalues and quasinormal modes}

Let us start by reviewing how we can write greybody factors in terms of monodromies [28, 34, 35, 37]. They informed reader eager to skip this technical discussion can find the main results for the Kerr-de Sitter greybody factor in (3.61), the quasinormal mode condition (3.63) and the angular eigenvalue condition (3.71).

A general solution of a second order linear ODE can be written as a linear combination of local solutions, such as those in (3.27) for the radial equation. Thus, we can define a vector of two general solutions by

$$
\Phi(r)=\Phi_{k}(r) g_{k}
$$

with $g_{k} \in \mathrm{SL}(2, \mathbb{C})$ constant matrices and $\Phi_{k}$ denotes a vector of local Frobenius solutions, with $k=1, \ldots, n$ referring to any of the $n$ singular points $r=r_{k}$ of the ODE. If we go around a positively-oriented loop $\gamma_{k}$ in the complex- $r$ plane enclosing only one of the singularities $\left(r_{k}\right)_{k=1, \ldots, n}$ (see figure 4 ), then $\Phi$ transforms to

$$
\Phi_{\gamma_{k}}(r)=\Phi(r) M_{k}
$$

where the initial value $\Phi$ of the solution at the loop is multiplied by a constant monodromy matrix $M_{k} \in \mathrm{SL}(2, \mathbb{C})$. 


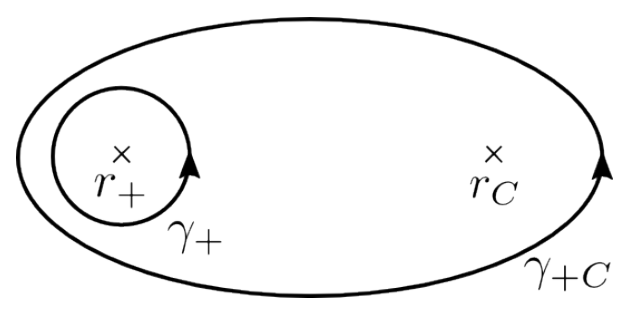

Figure 4. Example of local $\left(\gamma_{+}\right)$and composite $\left(\gamma_{+C}\right)$ monodromy paths in the complex- $r$ plane around the singular points $r=r_{+}$and $r_{C}$.

The matrices $g_{k}$ diagonalize the monodromy matrix $M_{k}$ if $2 \theta_{k} \notin \mathbb{Z}$, i.e.

$$
M_{k}=g_{k}^{-1}\left(\begin{array}{cc}
e^{2 \pi i \theta_{k}} & 0 \\
0 & e^{-2 \pi i \theta_{k}}
\end{array}\right) g_{k} .
$$

In principle, there should be an overall phase $e^{i \pi s}$ in (3.56), obtained from the common factor $\left(r-r_{k}\right)^{-s / 2}$ in the definition (3.27), but we absorb it, as a common overall factor, in the solution $\Phi$ to obtain $\operatorname{SL}(2, \mathbb{C})$ representations of the monodromy group. Notice that

$$
\operatorname{Tr} M_{k}=2 \cos \left(2 \pi \theta_{k}\right) .
$$

Given two singular points $r_{k}$ and $r_{j}$ with $j, k=1, \ldots, n$ and $j \neq k$, we wish to study the connection matrix

$$
\mathcal{M}_{j k}=g_{j} g_{k}^{-1},
$$

where the last equality follows from eq. (3.54). Thus, the connection matrix is constant and determined by the $g_{k}, k=1, \ldots, n$. Choosing a proper normalization for the local solutions, in terms of the $g_{k}$, such that $\operatorname{det} \mathcal{M}_{j k}=1$, we can write this connection matrix in the form (3.51).

Let us now choose a path $\gamma_{j k}$ which encloses two singular points $r_{j}$ and $r_{k}$, as in figure 4 , in such a way that the general solution changes to $\Phi_{\gamma_{j k}}(z)=\Phi(z) M_{j} M_{k}$. We define the composite monodromy coefficient $\sigma_{j k}$ by

$$
\operatorname{Tr}\left(M_{j} M_{k}\right)=-2 \cos \left(2 \pi \sigma_{j k}\right) .
$$

The reason for the extra minus sign here, in comparison with the definition of the single traces, is explained in appendix B. Applying eqs. (3.56), (3.58) and (3.59) to the case of (3.51), we find

$$
G_{s}=\overline{\mathcal{T}}_{s} \overline{\mathcal{T}}_{s}^{\star}=\frac{2 \sin \left(2 \pi \theta_{j}\right) \sin \left(2 \pi \theta_{k}\right)}{\cos \left(2 \pi\left(\theta_{j}-\theta_{k}\right)\right)+\cos \left(2 \pi \sigma_{j k}\right)} .
$$

This is the greybody factor for a connection matrix normalized as per eq. (3.51).

Quasinormal modes. For the radial equation (3.1b), according to (3.27) and (3.49), we can rewrite (3.60) as

$$
G_{s}(\omega, m)=\tau_{s} \mathcal{T}_{s}(\omega, m) \mathcal{T}_{-s}(-\omega,-m)=\frac{\sin \left(\pi\left(2 \theta_{+}-s\right)\right) \sin \left(\pi\left(2 \theta_{C}-s\right)\right)}{\cos \left(\pi\left(\sigma+\theta_{+}-\theta_{C}\right)\right) \cos \left(\pi\left(\sigma-\theta_{+}+\theta_{C}\right)\right)},
$$


where $\sigma \equiv \sigma_{+C}$. We wish to calculate QNM frequencies in Kerr-de Sitter. These frequencies are defined by the condition that the modes are purely ingoing at the black hole horizon and purely outgoing at the cosmological horizon. ${ }^{6}$ According to eq. (3.34), the QNM condition corresponds to $\left(\mathcal{T}_{s}\right)^{-1}=0$, i.e., poles of $\mathcal{T}_{s}$, which appears in eq. (3.61). The poles $\omega=\omega_{p}$ on the right hand side of eq. (3.61) are

$$
\sigma^{( \pm)}\left(\omega_{p}\right)= \pm\left(\theta_{C}-\theta_{+}\right)+N+\frac{1}{2}, \quad N \in \mathbb{Z}
$$

However, these poles may correspond to poles of $\tau_{s}$ or $\mathcal{T}_{s}^{\star}=\mathcal{T}_{-s}(-\omega,-m)$ as well. Not being able to distinguish between poles of $\mathcal{T}_{s}$ and those of $\mathcal{T}_{s}^{\star}$ or $\tau_{s}$ is a shortcoming of the monodromy formula above, as it only gives information about the product of these coefficients. However, below we recover the exact greybody factor in the rotating Nariai limit $r_{C} \rightarrow r_{+}$by using eq. (3.62). This will also allow us to identify the poles of $\mathcal{T}_{s}(\omega, m)$ with $N \in \mathbb{Z}^{+}$, and the poles of $\mathcal{T}_{-s}(-\omega,-m)$ with $N \in \mathbb{Z}^{-}$. While the former corresponds to the actual QNMs, the later corresponds to the reflected QNMs, and thus, in principle, they are not QNMs. In order to assess the possible poles coming from $\tau_{s}$, one needs to generalize the Teukolsky-Starobinsky identities obtained in ref. [47] for complex $\omega$. We leave this for future work.

In section 4, we are going to apply the APE derived in ref. [16] to find QNMs. Because the APE has the symmetry $\sigma \rightarrow-\sigma$, which is also compatible with eq. (3.60), changing the sign in front of $\left(\theta_{C}-\theta_{+}\right)$in eq. (3.62) is equivalent to letting $N \rightarrow-N-1$. Therefore, from the APE point of view, there is no distinction between the positive and negative $N$ possible poles in (3.62) and, from here on, we choose the QNM condition to be

$$
\sigma^{(+)}\left({ }_{s} \omega_{\ell m}\right)=\theta_{C}-\theta_{+}+N+\frac{1}{2}, \quad N \in \mathbb{Z}^{+} .
$$

As mentioned above, the condition $N \geq 0$ will be justified in the rotating Nariai limit in the next subsection. Finally, it is worth mentioning that, in this paper, we focus on the radial equation for which $2 \theta_{k} \notin \mathbb{Z}$, and so we do not cater for any potential QNM frequencies that could correspond to $2 \theta_{k} \in \mathbb{Z}$. This can introduce logarithm singularities in the solutions and thus change the QNM condition above.

Accumulation points of quasinormal modes. As a function of complex frequency, the transmission coefficient may have not only poles (QNMs) but also branch cuts. Apart from spurious (unphysical) branch cuts arising from the angular eigenvalue [52, 53], the transmission coefficient may also have cuts arising from the behaviour of the radial potential which may have physical consequences. Let us first write the radial equation $(3.1 \mathrm{~b})$ as $\left(\partial_{r_{*}}^{2}+\mathcal{V}(r)\right) \mathcal{R}(r)=0$, where $\mathcal{R}(r) \equiv \Delta_{r}^{s / 2}\left(r^{2}+a^{2}\right)^{1 / 2} R_{s, \omega, m}(r)$ and the radial potential $\mathcal{V}(r)$ is straightforwardly read-off from eq. (3.1b). It is easy to check that, if $r_{C} \neq r_{+}$, then $\mathcal{V}(r) \rightarrow \mathcal{V}_{+, C}+\mathcal{O}\left(e^{\alpha_{+}, C} r_{*}\right)$, as $r \rightarrow r_{+, C}$, for some constants $\mathcal{V}_{+, C}$ and $\alpha_{+}>0$ and $\alpha_{C}<0$ Based on the arguments of [54] (see also [55]), this asymptotic behaviour does not lead to branch points in the complex $\omega$-plane for the radial solutions which obey purely-outgoing (or purely-ingoing) boundary conditions at either $r_{+}$or $r_{C}$. On the other hand, in the case

\footnotetext{
${ }^{6}$ This is in contrast with Kerr, where the QNMs are purely outgoing at $r=\infty$.
} 
$r_{+}=r_{C}$, this singular point becomes an irregular singular point and it is easy to show that, then, $\mathcal{V}(r) \rightarrow \overline{\mathcal{V}}_{C}+\mathcal{O}\left(1 / r_{*}\right)$ as $r \rightarrow r_{+}=r_{C}$, for some constant $\overline{\mathcal{V}}_{C}$. This is essentially due to the fact that $r_{*}=\mathcal{O}\left(1 /\left(r-r_{+}\right)\right)$as $r \rightarrow r_{+}$when $r_{+}=r_{C}$, as opposed to $r_{*}=\mathcal{O}\left(\ln \left(r-r_{+}\right)\right)$ as $r \rightarrow r_{+, C}$ when $r_{+} \neq r_{C}$. Furthermore, $\Re\left(\sqrt{\overline{\mathcal{V}}_{C}}\right)$ possesses a zero at the superradiant bound frequency (namely, at the frequency such that $\tilde{\omega}_{C}=0$; recall that, in this extremal case, it is $\left.\tilde{\omega}_{+}=\tilde{\omega}_{C}\right)$. According to ref. [54], this asymptotic behaviour means that radial solutions obeying purely-outgoing (or purely-ingoing) boundary conditions at $r \rightarrow r_{+}$(see eq. (3.31)) possess a branch point at $\tilde{\omega}_{C}=\left(\tilde{\omega}_{+}=\right) 0$ when $r_{+}=r_{C} \cdot{ }^{7}$ Such branch point is carried over to the corresponding transmission coefficient $\left(\mathcal{T}_{s}, \mathcal{T}_{s}^{\star}, \tilde{\mathcal{T}}_{s}, \tilde{\mathcal{T}}_{s}^{\star}\right)$.

The above relationship between asymptotic behaviour of the radial potential and branch points in the complex $\omega$-plane is already well-known in Kerr. Indeed, the transmission coefficient in Kerr possesses a branch point at $\omega=0$, associated with the irregular singular point at $r=\infty$. This branch point gives rise to a power-law tail decay at latetimes $[52,56,57]$. More relevantly to the study here, in extremal $\left(r_{+}=r_{-}\right)$Kerr, the transmission coefficient has an extra branch point at $\tilde{\omega}_{+}=0$, which gives rise $[58,59]$ to the so-called Aretakis phenomenon at the horizon [60]. It has been shown [39, 41, 58, 61] that, in near-extremal Kerr, QNMs accumulate near $\tilde{\omega}_{+}=0$, effectively leading to a branch cut originating at $\tilde{\omega}_{+}=0$ in (exactly) extremal Kerr spacetime. We expect that a similar accumulation of QNMs occurs in Kerr-de Sitter both near $\tilde{\omega}_{+}=0$ and near $\tilde{\omega}_{C}=0$ as the extremal limit $r_{+} \rightarrow r_{C}$ is approached. In section 4.3, we show that this is indeed the case.

Renormalized angular momentum parameter. In ref. [11], the authors use a series of hypergeometric functions to express the solution of Heun equations. The hypergeometric functions depend on the so-called renormalized angular momentum parameter $\nu$. As discussed in [35], $\nu$ parametrizes the monodromy at infinity of the hypergeometric functions. Because the hypergeometric differential equation possesses only three regular singular points, we see that its monodromy at infinity is equivalent to its composite monodromy. In its turn, this composite monodromy of the hypergeometric differential equation is equal to the composite monodromy $\sigma_{01}$ of the full Heun equation modulo $2 n$, where $n \in \mathbb{Z}$. That results in the following relationship: $\sigma_{01}=2\left(\nu-\theta_{0}+\theta_{1}\right)+1(\bmod 2 n)$, where $n \in \mathbb{Z}$.

Angular eigenvalues. In order to find the angular eigenvalues ${ }_{s} \lambda_{\ell m}$, we need to impose a boundary condition on the solutions of the spin- $s$ angular equation (3.1a). We are interested in solutions which are regular in the domain $u \in[-1,1]$, corresponding to $z \in[0, x]$ in the Heun equation (3.17). It is well-known in the literature [11] that $(s, \ell, m)$ are either all integers or half-integers and that

$$
\ell \geq \max (|s|,|m|)
$$

Therefore, $2 \theta_{0}=m-s$ and $2 \theta_{x}=-m-s$ are integers and so the difference between Frobenius exponents for solutions at either $z=0$ or $z=x$ is an integer. This opens up the

\footnotetext{
${ }^{7}$ We note that the mentioned asymptotic behaviours of the radial potential are independent of the 'centrifugal' barrier.
} 
possibility of a logarithmic singularity in one of the local solutions at each singular point. According to standard Frobenius analysis $[14,36]$, the local solutions in this case are

$$
\begin{cases}S_{\text {reg }}^{(k)} \sim\left(u-u_{k}\right)^{\left|\theta_{k}\right|}, & u \rightarrow u_{k},(k=0, x), \\ S_{\text {irr }}^{(k)} \sim\left(u-u_{k}\right)^{-\left|\theta_{k}\right|}+A_{k}(u-1)^{\left|\theta_{k}\right|} \log \left(u-u_{k}\right), & \end{cases}
$$

for some coefficients $A_{k}$. If we define the vectors $S^{(k)} \equiv\left(S_{\mathrm{reg}}^{(k)} S_{\mathrm{irr}}^{(k)}\right)$, we find the following form for their monodromy matrices (recall eq. (3.55)) at, respectively, $z=0$ and $x$ :

$$
J_{0} \equiv e^{2 \pi i\left|\theta_{0}\right|}\left(\begin{array}{ll}
1 & 1 \\
0 & 1
\end{array}\right), \quad J_{x} \equiv e^{2 \pi i\left|\theta_{x}\right|}\left(\begin{array}{ll}
1 & 1 \\
0 & 1
\end{array}\right)
$$

We thus define a general solution of the master angular equation (3.1a) as

$$
S_{s, \ell, m}^{(\mathrm{reg})} \sim \begin{cases}A_{\mathrm{reg}} S_{\text {reg }}^{(x)}-A_{\mathrm{irr}} S_{\mathrm{irr}}^{(x)}, & u \rightarrow 1, \\ S_{\text {reg }}^{(0)}, & u \rightarrow-1,\end{cases}
$$

and

$$
S_{s, \ell, m}^{(\mathrm{irr})} \sim \begin{cases}\tilde{A}_{\mathrm{irr}} S_{\mathrm{irr}}^{(x)}-\tilde{A}_{\mathrm{reg}} S_{\text {reg }}^{(x)}, & u \rightarrow 1, \\ S_{\mathrm{irr}}^{(0)}, & u \rightarrow-1,\end{cases}
$$

for some coefficients $A_{\text {reg }}=A_{\text {reg }}(\omega, m)$ and $A_{\text {irr }}=A_{\text {irr }}(\omega, m)$, and similarly for the tilde connection coefficients. Therefore, in this angular case, we have a connection problem similar to the QNM one above. Given that the monodromy matrices of the general solution $\left(S_{s, \ell, m}^{(\mathrm{reg})} S_{s, \ell, m}^{(\mathrm{irr})}\right)=S^{(k)} g_{k}$ are given by $M_{k}=g_{k}^{-1} J_{k} g_{k}$, with $k=0, x$, we calculate $\operatorname{Tr}\left(M_{0} M_{x}\right)=2 \cos (2 \pi \sigma)$ using eqs. (3.51), (3.58), (3.59) and (3.66) to obtain

$$
A_{\text {irr }}= \pm \sqrt{2\left(1+(-1)^{|m-s|+|m+s|} \cos (2 \pi \sigma)\right)} .
$$

The regularity condition corresponds to choosing $A_{\text {irr }}=0$ in eq. (3.67), which is equivalent to

$$
\sigma=\ell+\frac{1}{2}, \quad \ell \in \mathbb{Z}, \text { if } \max (|s|,|m|) \in \mathbb{Z} ; \quad \ell \in \mathbb{Z}+\frac{1}{2}, \text { if } \max (|s|,|m|) \in \mathbb{Z}+\frac{1}{2} .
$$

As we are going to see in section 4 , the parameter $\ell$ introduced in eq. (3.70) is the same $\ell$ that labels the angular eigenvalues ${ }_{s} \lambda_{\ell m}$ and so the same as in eq. (3.64). Taking this into account, the condition eq. (3.70) together with the constraint (3.64) yield

$$
\sigma=\ell+\frac{1}{2}, \quad \ell \geq \max (|s|,|m|) .
$$

In the next section, we shall use the constraints (3.63) and (3.71) on the composite monodromy parameter $\sigma_{0 x}$ in order to find, respectively QNM frequencies and angular eigenvalues. 


\subsection{Quasinormal modes in the rotating Nariai limit}

In this subsection, we focus on the region between $r_{+}$and $r_{C}$ (thus discarding the other horizons) and study the extremal limit $r_{+} \rightarrow r_{C}$, which is also called the rotating Nariai limit [20, 32, 62]. The rotating Nariai limit is applied directly on the metric and it yields a new geometry which is topologically equivalent to $d S_{2} \ltimes S^{2}{ }^{8}$ Here we review this limit by finding the QNM frequencies of a scalar field which possesses general coupling with the curvature of this new extremal-limit geometry. This slightly extends the results in ref. [20] for minimal coupling to general coupling. We also show how to obtain the scalar radial equation of the rotating Nariai metric directly from the spin- $s$ master radial equation $(3.1 \mathrm{~b})$ of Kerr-de Sitter. The result for QNMs that we obtain in this limit here will be used as a check of the leading-order term in the higher-order expansion in the extremality parameter $\epsilon=\left(r_{C}-r_{+}\right) / L$ that we obtain in the next section.

The metric in the rotating Nariai limit can be obtained from the Kerr-de Sitter metric in eq. (2.1) via the following coordinate transformation: ${ }^{9}$

$$
\bar{t} \equiv \bar{\kappa} \epsilon t, \quad \bar{y} \equiv \frac{r-\bar{r}_{+}}{\epsilon L}, \quad \bar{\phi} \equiv \phi-\Omega_{+} t,
$$

where

$$
\bar{\kappa} \equiv \frac{\left(\bar{r}_{+}-\bar{r}_{-}\right)\left(3 \bar{r}_{+}+\bar{r}_{-}\right)}{\chi^{2} L\left(a^{2}+\bar{r}_{+}^{2}\right)}
$$

where $\left.\bar{r}_{ \pm} \equiv r_{ \pm}\right|_{\epsilon=0}$. The location of the cosmological horizon is $\bar{y}=1$. If we now take $\epsilon \rightarrow 0$ in the metric (2.1) after the coordinate change (3.72), we find [20]

$$
d s^{2}=\Gamma(\theta)\left(-\bar{y}(1-\bar{y}) d \bar{t}^{2}+\frac{d \bar{y}^{2}}{\bar{y}(1-\bar{y})}+\alpha(\theta) d \theta^{2}\right)+\gamma(\theta)(d \bar{\phi}+\bar{y} \bar{\Omega} d \bar{t})^{2}
$$

where $\bar{y} \in(0,1), \bar{\phi} \sim \bar{\phi}+2 \pi$ and

$$
\begin{aligned}
\Gamma(\theta) & \equiv \frac{\rho_{+}^{2} L}{\chi^{2} \bar{\kappa}\left(a^{2}+\bar{r}_{+}^{2}\right)}, & \alpha(\theta) & \equiv \frac{\chi^{2} \bar{\kappa}\left(a^{2}+\bar{r}_{+}^{2}\right)}{L \Delta_{\theta}}, \quad \gamma(\theta) \equiv \frac{\Delta_{\theta}\left(\bar{r}_{+}^{2}+a^{2}\right)^{2} \sin ^{2} \theta}{\rho_{+}^{2} \chi^{4}}, \\
\bar{\Omega} & \equiv \frac{2 a \bar{r}_{+} L}{\bar{\kappa}\left(a^{2}+\bar{r}_{+}^{2}\right)^{2}}, & \rho_{+}^{2} & \equiv \bar{r}_{+}^{2}+a^{2} \cos ^{2} \theta .
\end{aligned}
$$

Let us now consider a scalar field, with general coupling parameter $\xi$, propagating on the rotating Nariai geometry of eq. (3.74). The corresponding Klein-Gordon equation separates by variables and, for a field mode $e^{-i \bar{\omega} \bar{t}+i m \bar{\phi}} S_{m}(\theta) R_{\omega m}(\bar{y})$, with $\bar{\omega} \in \mathbb{C}$ and $m \in \mathbb{Z}$, the resulting angular and radial equations are, respectively,

$$
\begin{array}{r}
{\left[\frac{d}{d \theta}\left(\Delta_{\theta} \sin \theta\right) \frac{d}{d \theta}+\frac{m^{2} \chi^{4} \rho_{+}^{4}}{\left(\bar{r}_{+}^{2}+a^{2}\right) \Delta_{\theta} \sin \theta}+\left(\frac{12 \xi}{L^{2}} a^{2} \cos ^{2} \theta-\lambda_{\ell m}\right) \sin \theta\right] S_{m}(\theta)=0,} \\
{\left[\frac{d}{d \bar{y}} \bar{y}(\bar{y}-1) \frac{d}{d \bar{y}}+\frac{(\bar{\omega}+m \bar{\Omega} y)^{2}}{\bar{y}(\bar{y}-1)}+\bar{\lambda}_{\ell m}\right] R_{\omega m}(\bar{y})=0,}
\end{array}
$$

\footnotetext{
${ }^{8}$ The semi-direct product $\ltimes$ here means that the isometries of $d S_{2}$ must be accompanied by $S^{2}$ isometries so that they are isometries of the full spacetime.

${ }^{9}$ The definitions used in [20] can be recovered by letting $\epsilon \rightarrow \frac{r_{C}}{L} \epsilon$ and $\bar{\kappa} \rightarrow \frac{L}{\chi^{2} r_{C}} \bar{\kappa}$.
} 
where

$$
\bar{\lambda}_{\ell m} \equiv \frac{L^{2}}{\left(3 \bar{r}_{+}+\bar{r}_{-}\right)\left(\bar{r}_{+}-\bar{r}_{-}\right)}\left(\lambda_{\ell m}+\frac{12 \xi}{L^{2}} \bar{r}_{+}^{2}\right)
$$

and $\lambda_{\ell m}$ is the eigenvalue of the angular equation (3.77). Notice that (3.77) does not depend on the frequency $\bar{\omega}$. Therefore, the angular eigenvalue $\lambda_{\ell m}$ does not depend on $\bar{\omega}$ either.

The radial equation (3.78) has three regular singular points at $\bar{y}=0,1$ and $\infty$ and can be transformed to the hypergeometric equation [20]

$$
\left[\frac{d^{2}}{d \bar{y}^{2}}+\left(\frac{C}{\bar{y}}+\frac{A+B+1-C}{\bar{y}-1}\right) \frac{d}{d \bar{y}}+\frac{A B}{\bar{y}(\bar{y}-1)}\right] \bar{f}_{\omega m}=0
$$

where $\bar{f}_{\omega m}(\bar{y}) \equiv \bar{y}^{(1-C) / 2}(\bar{y}-1)^{(C-A-B) / 2} R_{\omega m}(\bar{y})$, with parameters

$$
A \equiv \frac{1+i \beta}{2}+i m \bar{\Omega}, \quad B \equiv \frac{1-i \beta}{2}+i m \bar{\Omega}, \quad C \equiv 1-2 i \bar{\omega},
$$

and

$$
\beta \equiv \sqrt{4\left(m^{2} \bar{\Omega}^{2}+\bar{\lambda}_{\ell m}\right)-1} .
$$

The Frobenius exponents of (3.78) $\theta_{0,1, \infty}$ at, respectively, $\bar{y}=0,1$ and $\infty$ can be found from these parameters to be given via

$$
2 \theta_{1}=C-A-B=-2 i(\bar{\omega}+m \bar{\Omega}), \quad 2 \theta_{0}=1-C=2 i \bar{\omega}, \quad 2 \theta_{\infty}=A-B=i \beta .
$$

Let $R_{\text {in }}$ be a solution of (3.78) defined via the boundary condition

$$
R_{\text {in }} \sim \begin{cases}(\bar{y})^{-\theta_{0}} & \text { as } \quad \bar{y} \rightarrow 0, \\ A_{\ell m}^{(\text {out })}(1-\bar{y})^{-\theta_{1}}+A_{\ell m}^{(\text {in })}(1-\bar{y})^{\theta_{1}} & \text { as } \quad \bar{y} \rightarrow 1,\end{cases}
$$

for some coefficients $A_{\ell m}^{(\text {out })}$ and $A_{\ell m}^{(\text {in })}$. Assuming that $\Re \bar{\omega} \geq 0$, eq. (3.84) represents scattering in the region $\bar{y} \in(0,1)$ with a purely ingoing condition at $\bar{y}=0$, while the notion of ingoing and outgoing at $\bar{y}=1$ depends on the sign of $\Re(\bar{\omega}+m \bar{\Omega})$. According to the asymptotic behaviour of hypergeometric functions [63], we have

$$
\begin{aligned}
A_{\ell m}^{(\mathrm{in})} & =\frac{\Gamma(1-2 i \bar{\omega}) \Gamma(-2 i(\bar{\omega}+m \bar{\Omega}))}{\Gamma((1+i \beta) / 2-i m \bar{\Omega}-2 i \bar{\omega}) \Gamma((1-i \beta) / 2-i m \bar{\Omega}-2 i \bar{\omega})}, \\
A_{\ell m}^{(\mathrm{out})} & =\frac{\Gamma(1-2 i \bar{\omega}) \Gamma(2 i(\bar{\omega}+m \bar{\Omega}))}{\Gamma((1-i \beta) / 2+i m \bar{\Omega}) \Gamma((1+i \beta) / 2+i m \bar{\Omega})} .
\end{aligned}
$$

We find the QNM frequencies by requiring that $A_{\ell m}^{(\mathrm{in})}=0$ in the case $\Re(\bar{\omega}+m \bar{\Omega})>0 .{ }^{10}$ This condition straightforwardly yields that the QNM frequencies $\bar{\omega}=\bar{\omega}_{N \ell m}^{ \pm}$are given by

$$
2 \bar{\omega}_{N \ell m}^{ \pm}=-i\left(N+\frac{1}{2}\right)-m \bar{\Omega} \pm \sqrt{\bar{\lambda}_{\ell m}+m^{2} \bar{\Omega}^{2}-\frac{1}{4}}, \quad N=0,1,2, \ldots
$$

\footnotetext{
${ }^{10}$ For $\Re(\bar{\omega}+m \bar{\Omega})<0$, we should instead look for zeros of $A_{\ell m}^{(\text {out })}$, but it does not possess any non-trivial zeros.
} 
The greybody factor for real frequencies follows readily from eq. (3.85):

$$
G(\bar{\omega}, m)=1-\left|\frac{A_{\ell m}^{(\mathrm{out})}}{A_{\ell m}^{\text {(in })}}\right|^{2}=\frac{2 \sinh (2 \pi \bar{\omega}) \sinh (2 \pi(\bar{\omega}+m \bar{\Omega}))}{\cosh (2 \pi(2 \bar{\omega}+m \bar{\Omega}))+\cosh (\pi \beta)} .
$$

Eqs. (3.87) and (3.88) agree with [20] for $\xi=0$ (eq. (3.87) also agrees with the QNM frequencies of the geometry resulting from the extremal Nariai limit $r_{C} \rightarrow r_{+}$of Schwarzschildde Sitter spacetime [64] by setting $a=0, \xi=0, r_{-}=0$ and $\left.\lambda_{\ell m}=\ell(\ell+1)\right) .{ }^{11}$ Eqs. (3.87) and (3.88) are new results for the nonminimal coupling case $(\xi \neq 0)$.

Alternatively, the result (3.88) can be derived easily if we use the monodromy technique $[34,35]$ reviewed in the previous section. The greybody factor is given by eq. (3.60) with $\sigma$ replaced by $\theta_{\infty}$ (as explained below):

$$
\begin{aligned}
G(\bar{\omega}, m) & =\frac{2 \sin \left(2 \pi \theta_{0}\right) \sin \left(2 \pi \theta_{1}\right)}{\cos \left(2 \pi\left(\theta_{0}-\theta_{1}\right)\right)+\cos \left(2 \pi \theta_{\infty}\right)} \\
& =\frac{2 \sinh (2 \pi \bar{\omega}) \sinh (2 \pi(\bar{\omega}+m \bar{\Omega}))}{\cosh (2 \pi(2 \bar{\omega}+m \bar{\Omega}))+\cosh (\pi \beta)},
\end{aligned}
$$

which is exactly the same result as eq. (3.88). Therefore, we can avoid the calculation of the exact connection coefficients using the general formula (3.60).

The poles of the greybody factor eq. (3.89) are given by (3.87) but with $N \in \mathbb{Z}$ instead of $N=0,1,2, \ldots$ This is an intrinsic limitation of finding QNMs from the greybody factor, as the two possibilities of $N \in \mathbb{Z}^{+}$and $N \in \mathbb{Z}^{-}$appear here because we take the ratio of connection coefficients in (3.88). From the exact connection coefficients (3.85), we see that positive $N$ corresponds to $\bar{\omega}, m>0$ and negative $N$ corresponds to $\bar{\omega}, m<0$. The transformation $\{\bar{\omega}, m\} \rightarrow\{-\bar{\omega},-m\}$ is a symmetry of the radial equation (3.78) but changes the notion of ingoing and outgoing solutions. We can decide which branch to take by going back to the solutions (3.84).

Spin- $s$ quasinormal modes from the Kerr-de Sitter master equation. The formula (3.89) deserves further explanation. Firstly, notice that (3.60) is valid for a generic connection problem between two singular points of a Fuchsian equation which possesses any number of singular points, as long as the monodromy matrices are diagonalizable. Secondly, as the hypergeometric equation is a Fuchsian equation with three regular singular points, the composite monodromy around $\bar{y}=0$ and $\bar{y}=1$ must be equal to the monodromy $\theta_{\infty}$ at $\bar{y}=\infty$. This can be made even more precise by noticing that we can recover the rotating Nariai radial equation (3.78) for $\xi=1 / 6$ in canonical form directly from the full Heun equation in Kerr-de Sitter (3.25). Instead of the coordinate choice (3.22), we choose

$$
\begin{gathered}
z(r)=\zeta_{\infty} \frac{r-r_{+}}{r-r_{--}}, \quad \zeta_{\infty}=\frac{2 r_{C}+r_{+}+r_{-}}{r_{C}-r_{+}}, \quad x=\zeta_{\infty} \frac{r_{-}-r_{+}}{2 r_{-}+r_{C}+r_{-}}, \\
r=\left(r_{+}, r_{C}, r_{-}, r_{--}, \infty\right) \quad \mapsto \quad z=\left(0,1, x, \infty, \zeta_{\infty}\right) .
\end{gathered}
$$

\footnotetext{
${ }^{11}$ When comparing, one should taking into account the different coordinate times.
} 
This means that, with respect to the monodromies in eq. (3.20), we make the replacement

$$
\left(\theta_{0}, \theta_{1}, \theta_{x}, \theta_{\infty}\right) \mapsto\left(\theta_{1}, \theta_{x}, \theta_{0}, \theta_{\infty}\right)
$$

Both $\zeta_{\infty}$ and $x$ diverge in the extremal limit $r_{+} \rightarrow r_{C}$ as $\epsilon^{-1}$, and this implies that $\theta_{0}$ and $\theta_{1}$ diverge as $x \rightarrow \infty$. We can regularize the monodromies in eq. (3.20) by defining

$$
\bar{\omega} \equiv \frac{\omega-m \Omega_{+}}{\bar{\kappa} \epsilon},
$$

which gives, for small $\epsilon$ and fixed $\bar{\omega}$,

$$
\begin{aligned}
\theta_{0} & =\bar{\theta}_{0}+\mathcal{O}(\epsilon), & \theta_{1} & =\bar{\theta}_{1}+\mathcal{O}(\epsilon), \\
\theta_{x} & =i m \bar{\Omega} \frac{\left(3 \bar{r}_{+}+\bar{r}_{-}\right)}{4 \bar{r}_{+}}+\frac{s}{2}+\mathcal{O}(\epsilon), & \theta_{\infty} & =i m \bar{\Omega} \frac{\left(\bar{r}_{+}-\bar{r}_{-}\right)}{4 \bar{r}_{+}}+\frac{s}{2}+\mathcal{O}(\epsilon),
\end{aligned}
$$

with

$$
\bar{\theta}_{0} \equiv i \bar{\omega}+\frac{s}{2}, \quad \bar{\theta}_{1} \equiv-i(\bar{\omega}+m \bar{\Omega})+\frac{s}{2},
$$

where the leading order terms should be calculated at $\epsilon=0$. The accessory parameter $K_{x}$ in (A.40), with the appropriate identifications of eqs. (3.90) and (3.91), goes to zero as $x \rightarrow \infty$, but $x(x-1) K_{x} /(z-x)$ is finite. Applying this limit (i.e., $x \rightarrow \infty-$ or, equivalently, $\epsilon \rightarrow 0-$ with $z$ finite) to the Kerr-de Sitter eq. (3.25) yields

$$
\left[\frac{d^{2}}{d z^{2}}+\left(\frac{1-2 \bar{\theta}_{0}}{z}+\frac{1-2 \bar{\theta}_{1}}{z-1}\right) \frac{d}{d z}+\frac{{ }_{s} \bar{\lambda}_{\ell m}-2 s+i m(1-2 s) \bar{\Omega}}{z(z-1)}\right] f=0,
$$

and

$$
{ }_{s} \bar{\lambda}_{\ell m} \equiv \frac{L^{2}}{\left(3 \bar{r}_{+}+\bar{r}_{-}\right)\left(\bar{r}_{+}-\bar{r}_{-}\right)}\left(\left.{ }_{s} \lambda_{\ell m}\right|_{\epsilon \rightarrow 0}-s\left(1-\frac{a^{2}}{L^{2}}\right)+\frac{2}{L^{2}}(2 s+1)(s+1) \bar{r}_{+}^{2}\right),
$$

where we remind the reader that ${ }_{s} \lambda_{\ell m}$ is the Kerr-de Sitter angular eigenvalue. One can check that by taking the rotating Nariai limit on the master angular equation (3.77) in Kerr-de Sitter then, for $s=0$ and $\xi=1 / 6$, one recovers eq. (3.77) with $\left.{ }_{0} \lambda_{\ell m}\right|_{\epsilon \rightarrow 0}$ in the place of $\lambda_{\ell m}$ (and so they are equal when imposing the eigenvalue condition). Therefore, eq. (3.96) matches eq. (3.79) for $s=0$ and $\xi=1 / 6$. The reason for this relationship is that eq. (3.95) is equivalent to eq. (3.78) with $z=\bar{y}$ after an appropriate homotopic transformation (see appendix A for the general discussion). If we go to the next order in $\epsilon$ in the full master radial equation, we can start to calculate corrections to the extremal QNM result in eq. (3.87). That is what we achieve in the next section.

\section{Accessory parameter expansion}

In the previous section, we considered an arbitrary second order Fuchsian ODE and we expressed the greybody factor (written as a product of connection coefficients) in terms of the monodromies of local solutions - see eq. (3.60). By looking for poles of this formula we found condition (3.62) for $\sigma_{+C}$, which sets the boundary condition for QNMs (3.63)of the 
Kerr-dS master radial equation for $N \in \mathbb{Z}^{+}$. We also similarly discussed the monodromy condition (3.71) for angular eigenvalues. We thus mapped special boundary conditions for a solution of the radial or angular equation to constraints on its monodromy exponents. This approach was proposed for the confluent Heun equation in $[34,37]$ and for the Heun equation in $[28,35]$. We have in fact shown above that this method works in the particular case of the rotating Nariai limit. This limit yields a simpler case since the composite monodromy then reduces to a local monodromy of the hypergeometric equation. In this section, we apply the composite monodromy constraints to solutions of the Heun equation (3.5) obtained from the Kerr-de Sitter master equation. Below we present our results using the APE and the numerical checks done using Leaver's method.

\subsection{How to obtain the APE}

Here we review the mathematical background behind the APE and its derivation done in [16]. The monodromy group of functions on the complex plane with four regular singular points are labeled by six parameters [16]. Apart from the four monodromies $\boldsymbol{\theta}=\left(\theta_{0}, \theta_{1}, \theta_{x}, \theta_{\infty}\right)$ at the singular points, there are two extra composite monodromies, $\left(\sigma_{0 x}, \sigma_{1 x}\right)$. We thus define the monodromy data of the four-punctured sphere as the six parameters $\mathcal{M}_{\mathrm{SL}(2, \mathbb{C})} \equiv\left(\theta_{0}, \theta_{1}, \theta_{x}, \theta_{\infty} ; \sigma_{0 x}, \sigma_{1 x}\right)$. This means that the Heun equation is not the most general Fuchsian equation with four regular singular points, as it has only 5 parameters $\mathcal{M}_{\text {Heun }}=\left(\theta_{0}, \theta_{1}, \theta_{x}, \theta_{\infty} ; K_{x}\right)$ (see below eq. (3.10)). Instead, we can say that $\mathcal{M}_{\text {Heun }}$ corresponds to a reduced case of $\mathcal{M}_{\mathrm{SL}(2, \mathbb{C})}$ with only one composite monodromy, say, $\mathcal{M}_{\text {red }} \equiv\left(\theta_{0}, \theta_{1}, \theta_{x}, \theta_{\infty} ; \sigma_{0 x}, \sigma_{1 x}=\sigma_{1 x}\left(\sigma_{0 x}\right)\right)$. Then, $\mathcal{M}_{\text {red }}$ can be mapped to $\mathcal{M}_{\text {Heun }}$ via the relation $K_{x}=K_{x}\left(\boldsymbol{\theta}, \sigma_{0 x}, x\right)$. For fixed $x$, the APE provides the Riemann-Hilbert map between $\mathcal{M}_{\text {red }}$ and $\mathcal{M}_{\text {Heun }}$. The small-x expansion of this relation is called the accessory parameter expansion (APE). In ref. [16], the APE was obtained using isomonodromic deformations, as we review below. For other approaches to obtaining the APE, we refer the reader to, for example, $[15,18,19]$. For recent numerical results on finding $2 \mathrm{D}$ conformal maps from the APE using the approach of [16, 28], see [17].

The APE obtained in [16] depends on monodromy data $\mathcal{M}_{\text {red }}$ which is independent of $x$. This is contrary to what happens in the case of the ODEs satisfied by perturbations of Kerr-de Sitter black holes. In the case of the radial ODE in Kerr-de Sitter, $x \propto \epsilon$ and the monodromies diverge for small $\epsilon$. However, as discussed in section 3 , if we fix the frequency $\bar{\omega}$ defined in eq. (3.92), the monodromies converge as an expansion in $\epsilon$ and the accessory parameter is analytic for small $\epsilon$. Therefore, by setting $\sigma \equiv \sigma_{0 x}$ and expanding all parameters $(x(\epsilon), \boldsymbol{\theta}(\epsilon), \sigma(\epsilon))$ in terms of $\epsilon$ as

$$
\begin{aligned}
x & =\epsilon\left(x_{0}+\epsilon x_{1}+\ldots\right), & & \sigma=\sigma_{0}+\epsilon \sigma_{1}+\ldots, \\
\theta_{k} & =\theta_{k, 0}+\epsilon \theta_{k, 1}+\ldots, & & k \in \mathcal{J},
\end{aligned}
$$

we obtain the APE

$$
K_{x}=\frac{K_{x,-1}}{\epsilon}+\sum_{n=0}^{\infty} K_{x, n} \epsilon^{n},
$$

for some coefficients $x_{j}$ and $\theta_{k, j}$ and functions $K_{x, n}=K_{x, n}\left(x_{j}, \theta_{k, j}, \sigma_{j}\right), j \geq 0, n \geq-1$, $k=0,1, x, \infty$. 
In the case of the angular ODE, we have $x \propto \alpha$ and we set $\epsilon=\alpha$. The monodromies in eq. (3.12) converge for small $\alpha$ and fixed $L$ and we obtain the APE for the angular eigenvalue from eq. (4.2). In the case of QNMs, we instead set $\epsilon=\left(r_{C}-r_{+}\right) / L$ and use the frequency definition in eq. (3.92), which gives a regular expansion for the monodromies as $\epsilon \rightarrow 0$ for fixed $\bar{\omega}$. Given the APE expansions in both cases, we can equate it with the exact accessory parameters coming from the angular and radial ODEs (see, respectively, eqs. (3.18) and (3.26)), using the exact monodromies $\boldsymbol{\theta}$ and the QNM condition eq. (3.63) and the angular eigenvalue condition eq. (3.71). This procedure, which we shall present in more detail below, will give the expansions in eqs. (1.1) and (1.3). First, however, we shall show how to obtain the APE from a technique called isomonodromic deformation [65-67].

The most general monodromy data $\mathcal{M}_{\mathrm{SL}(2, \mathbb{C})}$ for an ODE with four singular points includes two composite monodromies, $\left(\sigma_{0 x}, \sigma_{1 x}\right)$. However, as we commented above, the Heun equation (3.5) has only one extra parameter $K_{x}$. To account for these two composite monodromies, we write the most general Fuchsian equation as

$$
\begin{aligned}
& y^{\prime \prime}+\left(\frac{1-2 \theta_{0}}{z}+\frac{1-2 \theta_{1}}{z-1}+\frac{2-2 \theta_{x}}{z-t}-\frac{1}{z-\lambda(t)}\right) y^{\prime}+ \\
& +\left(\frac{b_{1} b_{2}}{z(z-1)}-\frac{t(t-1) K\left(\theta_{0}, \theta_{1}, \theta_{x}-\frac{1}{2}, \theta_{\infty} ; \lambda(t), \mu(t), t\right)}{z(z-1)(z-t)}+\frac{\lambda(t)(\lambda(t)-1) \mu(t)}{z(z-1)(z-\lambda(t))}\right) y=0,
\end{aligned}
$$

where $\theta_{\infty}=b_{1}-b_{2}, \lambda(t)$ and $\mu(t)$ are some $t$-dependent functions with $t \in \mathbb{C}$ and $^{12}$

$$
\begin{aligned}
K\left(\theta_{0}, \theta_{1}, \theta_{x}-\right. & \left.\frac{1}{2}, \theta_{\infty} ; \lambda, \mu, t\right) \\
& \equiv \frac{\lambda(\lambda-1)(\lambda-t)}{t(t-1)}\left[\mu^{2}-\left(\frac{2 \theta_{0}}{\lambda}+\frac{2 \theta_{1}}{\lambda-1}+\frac{2 \theta_{x}-2}{\lambda-t}\right) \mu+\frac{b_{1} b_{2}}{\lambda(\lambda-1)}\right] .
\end{aligned}
$$

The form of (4.4) follows by the requirement that $\lambda(t)$ is an apparent singularity, i.e., the solution has no branch cut at this point. The ODE parameters can be mapped to $\left(\sigma_{0 x}, \sigma_{1 x}\right)$ via initial conditions for the isomonodromic flow $(\lambda(t), \mu(t))$, whose Hamiltonian is given by eq. (4.4). However, there is a special set of initial conditions at $t=x[28,35]$ that reduce the general eq. (4.3) to the canonical Heun equation (3.5) with the same monodromy data $\mathcal{M}_{\text {Heun }}$. These initial conditions are given by

$$
t=x, \quad \lambda(x)=x, \quad \mu(x)=-\frac{K_{x}}{2 \theta_{x}-1} .
$$

In this case, we reduce the number of parameters of the ODE from two $(\lambda, \mu)$ to only one $\left(K_{x}\right)$, and similarly for the composite monodromies, since we have $\sigma_{1 x}=\sigma_{1 x}\left(\sigma_{0 x}, x\right)$. This is explained in full detail in ref. [16]. Here we just report on the solution for the accessory parameter.

The main difference between the current work and [16] is that here we want $K_{x}$ as an expansion in $\epsilon$ using the expansion of the monodromy data in (4.1), as described above.

\footnotetext{
${ }^{12}$ The parameter $\lambda$ here is unrelated to the angular eigenvalue ${ }_{s} \lambda_{\ell m}$; these are the symbols typically used in the literature of isomonodromic deformations.
} 
This changes the expansion obtained in [16], which becomes more complicated. It is more convenient to carry out the expansion for the accessory parameter of the ODE in the normal form (see appendix A for definitions)

$$
\mathcal{H}_{x} \equiv K_{x}+\frac{\left(1-2 \theta_{0}\right)\left(1-2 \theta_{x}\right)}{2 x}+\frac{\left(1-2 \theta_{1}\right)\left(1-2 \theta_{x}\right)}{2(x-1)} .
$$

This is the form most straightforwardly connected to 2D CFT. Using the procedure outlined in appendix $\mathrm{C}$, we find

$$
\begin{aligned}
\mathcal{H}_{x}= & \frac{-4 \theta_{0,0}^{2}-4 \theta_{x, 0}^{2}+4 \sigma_{0}^{2}+1}{4 x_{0} \epsilon}+\frac{\left(-4 \theta_{\infty, 0}^{2}+4 \theta_{1,0}^{2}+4 \sigma_{0}^{2}-1\right)\left(-4 \theta_{0,0}^{2}+4 \theta_{x, 0}^{2}+4 \sigma_{0}^{2}-1\right)}{32 \sigma_{0}^{2}-8} \\
& +\frac{x_{1}\left(4 \theta_{0,0}^{2}+4 \theta_{x, 0}^{2}-4 \sigma_{0}^{2}-1\right)+8 x_{0}\left(-\theta_{0,0} \theta_{0,1}-\theta_{x, 0} \theta_{x, 1}+\sigma_{0} \sigma_{1}\right)}{4 x_{0}^{2}}+\mathcal{O}(\epsilon),
\end{aligned}
$$

where we made use of the expansions in eqs. (4.1). We have calculated this series up to order $\epsilon^{3}$ in order to compare with the numerical results, but the higher-order terms are particularly long so we do not display them here.

The key point is that now we can equate two small- $\epsilon$ expansions for $\mathcal{H}_{x}$. One is the APE (4.7). The other expansion is obtained from eq. (4.6) with the accessory parameter given by eq. (3.26) or (3.18) in, respectively, the radial or angular cases, and expanded for small $\epsilon$. We call the equality between these two small- $\epsilon$ expansions for $\mathcal{H}_{x}$ the "APE equation". We shall next proceed to do this, first for the angular case and then for the radial case.

\subsection{Angular eigenvalues}

Here we wish to apply the APE in eq. (4.7) to find the angular eigenvalues ${ }_{s} \lambda_{\ell m}$ in eq. (3.1a). The expansion parameter in APE here is $\epsilon=\alpha=a / L$ and we expand in $\alpha$ only after replacing $a$ by $\alpha L$ in the monodromies in eq. (3.12). Since $a$ in eq. (3.12) only appears in the combination $a \omega / \alpha$, the mentioned expansion procedure is equivalent to expanding for both small $\alpha$ and small $a \omega$ (without the replacement of $a$ by $\alpha L$ ), both quantities being of the same order in smallness. From the APE point of view, we expand it up to order $\alpha^{3}$, which, from the previous observation, gives all powers $\alpha^{n}(a \omega)^{m}$ for $n+m=0, \ldots, 3$ of ${ }_{s} \lambda_{\ell m}(a \omega, \alpha)$, as in (1.2).

The monodromies (3.12) are polynomial in $\alpha$ (after replacing $a$ by $\alpha L$ ) and we expand $x$ to one order higher in $\alpha$

$$
x=\frac{4 i \alpha}{(i+\alpha)^{2}}=-4 i \alpha+8 \alpha^{2}+12 i \alpha^{3}-16 \alpha^{4}+O\left(\alpha^{5}\right)
$$

Using eqs. (3.18), (4.6) and (4.8) we obtain the following expansion for the accessory parameter:

$$
\begin{aligned}
x(x-1) \mathcal{H}_{x}= & \left(4 i \alpha^{3}+3 \alpha^{2}-2 i \alpha-1\right){ }_{s} \lambda_{\ell m}+\alpha^{3}\left(4 L \omega(m-s)-2 i m^{2}-10 i m s+6 i\right) \\
& +2 \alpha^{2}(-2 i L \omega(m-s)-m(m+3 s)+2)+2 \alpha(L \omega(s-m)+i(m(m+s)-1))+ \\
& +\frac{m^{2}-s^{2}-1}{2}+O\left(\alpha^{4}\right) .
\end{aligned}
$$


Equating this with the APE (4.7), using the monodromies (3.12) and the angular eigenvalue condition (3.71) yields the APE equation in the angular case. We then use this APE equation to isolate for ${ }_{s} \lambda_{\ell m}$, thus yielding an expression which we expand for small $\alpha$. We thus obtain eq. (1.3), where the explicit form of the coefficients $\lambda_{\omega, k}$ expanded for small $\alpha$ is given in appendix E. We have checked that this result matches (in the orders contained in both expansions) the angular eigenvalue expansion up to order $\left(\alpha^{2}, \omega^{2}\right)$ calculated in [11]. We have also checked that, in the Kerr limit $L \rightarrow \infty$, all the orders that we give agree with the small-aw expansion given in [22]. Thus, the eigenvalue expansion that we have obtained already exists in the literature (split between [11] and [22]) — the derivation here serves to validate the APE and provides a way of extending the expansion to higher orders.

\subsection{Quasinormal mode frequencies}

Let us now calculate the QNM frequencies in Kerr-de Sitter around the rotating Nariai limit using the APE. This calculation is more complicated than in the angular eigenvalue case, as both sides of the APE equation depend explicitly on powers of the frequency $\omega$. Therefore, we cannot just isolate $\omega$ as we did for the angular eigenvalue in the angular case. However, as mentioned above, the QNM frequency can still be calculated order-by-order in $\epsilon$ as displayed in eq. (1.1). We show here the explicit calculation near the rotating Nariai limit and just display the result up to order $\epsilon$. We, in fact, obtained the expansion up to order $\left(\epsilon^{3}, a^{3}\right)$ and provide it in appendix D. We numerically-checked the higher order expansion, which is discussed in the next section.

In this section, we will use the change of coordinates given by eq. (3.22), where, in particular, $r=r_{C}$ is mapped to $z=0$ and $r=r_{+}$is mapped to $z=x$. The expansion parameter here is $\epsilon=\left(r_{C}-r_{+}\right) / L$. Therefore,

$$
x=\left(\frac{2 r_{-}+r_{+}+r_{C}}{2 r_{+}+r_{C}+r_{-}}\right) \frac{r_{C}-r_{+}}{r_{C}-r_{-}} \sim \frac{2\left(\bar{r}_{+}+\bar{r}_{-}\right)}{\left(3 \bar{r}_{+}+\bar{r}_{-}\right)\left(\bar{r}_{+}-\bar{r}_{-}\right)} L \epsilon \quad \text { as } \quad r_{C} \rightarrow r_{+} .
$$

So the extremal limit $r_{C} \rightarrow r_{+}$corresponds to $x \rightarrow 0$. We expand the monodromies (3.20) by expressing $\omega=m \Omega_{+}+\epsilon \bar{\kappa} \bar{\omega}$ and expanding for small $\epsilon$ with $\bar{\omega}$ fixed:

$$
\begin{array}{lll}
\theta_{0}=-i(\bar{\omega}+m \bar{\Omega})+\frac{s}{2}+\mathcal{O}(\epsilon), & \theta_{1}=i m \bar{\Omega} \frac{\left(3 \bar{r}_{+}+\bar{r}_{-}\right)}{4 \bar{r}_{+}}+\frac{s}{2}+\mathcal{O}(\epsilon), \\
\theta_{x}=i \bar{\omega}+\frac{s}{2}+\mathcal{O}(\epsilon), & \theta_{\infty}=i m \bar{\Omega} \frac{\left(\bar{r}_{+}-\bar{r}_{-}\right)}{4 \bar{r}_{+}}+\frac{s}{2}+\mathcal{O}(\epsilon),
\end{array}
$$

where $\bar{\Omega}$ is defined in eq. (3.76) and $\bar{\kappa}$ in eq. (3.73).

We now set out to obtain the APE equation. First, we expand

$$
\bar{\kappa} \bar{\omega}=\bar{\omega}_{0}+\bar{\omega}_{1} \epsilon+\bar{\omega}_{2} \epsilon^{2}+\mathcal{O}\left(\epsilon^{3}\right)
$$

for some coefficients $\bar{\omega}_{0,1,2}$ which we set out to determine. In order to obtain one of the sides of the APE equation, we use eq. (4.6) for $\mathcal{H}_{x}$ with the accessory parameter given by eq. (3.26) consistently expanded for small $\epsilon$. For this expansion, we use eq. (4.11) for the monodromies and eq. (4.12) for the frequency, which in its turn is also used for expanding the angular eigenvalue in $\epsilon$. 
The other side of the APE equation is the APE (4.7). This expression depends on the coefficients of the expansion of the monodromies as well as on $\sigma$, for which we use the QNM condition given by eq. (3.63). In order to obtain the final expansion for $\mathcal{H}_{x}$ from the APE, because of the replacement in eq. (3.63), the monodromies need to be expanded as per eq. (4.11) and the frequency needs to be expanded as per eq. (4.12).

We have now explained how to obtain the two sides of the radial APE equation. This APE equation gives polynomial equations for the coefficients of $\bar{\omega}$ at each order in $\epsilon$. The first coefficient $\bar{\omega}_{0}$ obeys a quadratic equation and the other $\omega_{1,2, \ldots}$ obey linear equations, depending on the previously obtained ones in a recursive way. We next proceed to give the explicit expression for $\bar{\omega}$ to leading order in $\epsilon$, which coincides with the rotating Nariai limit result.

The first side of the APE equation, which is obtained from eqs. (3.26), (4.6), (4.11) and (4.12), reads, to leading order in $\epsilon$,

$$
\begin{aligned}
x(x-1) \mathcal{H}_{x} & ={ }_{s} \bar{\lambda}_{\ell m}-2 s^{2}+2 s \theta_{x}+2 \theta_{0}\left(s-\theta_{x}\right)-s-\frac{1}{2}+\mathcal{O}(\epsilon) \\
& =-2\left(\bar{\omega}_{0}\right)^{2}-2 m \bar{\Omega} \bar{\omega}_{0}+{ }_{s} \bar{\lambda}_{\ell m}-s(1+i m \bar{\Omega})-\frac{s^{2}+1}{2}+\mathcal{O}(\epsilon),
\end{aligned}
$$

where ${ }_{s} \bar{\lambda}_{\ell m}$ is given in eq. (3.96). Equating the expansion for $\mathcal{H}_{x}$ coming from (4.13) with the one coming from the APE (4.7) as explained above, yields, to leading order, the following quadratic equation for $\bar{\omega}_{0}$ :

$$
\left(\frac{\bar{\omega}_{0}}{\bar{\kappa}}\right)^{2}+(m \bar{\Omega}+i(N+1 / 2)) \frac{\bar{\omega}_{0}}{\bar{\kappa}}+\frac{1}{4}\left({ }_{s} \bar{\lambda}_{\ell m}-2 i m \bar{\Omega}(N+1 / 2)+N(N+1)-s(s+1)\right)=0,
$$

with $N \in \mathbb{Z}$ (which comes from the QNM condition in eq. (3.63)). The two solutions of this equation are

$$
\frac{2 \bar{\omega}_{0}}{\bar{\kappa}}=-i\left(N+\frac{1}{2}\right)-m \bar{\Omega} \pm \sqrt{{ }_{s} \bar{\lambda}_{\ell m}+m^{2} \bar{\Omega}^{2}-s(s+1)-\frac{1}{4}} .
$$

This expression agrees with eq. (3.87) for $s=0$ and $\xi=1 / 6$. As dictated by eq. (3.87), from now on $N$ is restricted to be a non-negative integer - we note that the APE method does not determine the sign of $N$, this is just imposed via an independent calculation (namely, the one that led to eq. (3.87)). ${ }^{13}$

Putting together eqs. (3.92), (4.12) and (4.15), we have that the near-extremality expansion for the QNM frequencies in Kerr-de Sitter is:

$$
{ }_{s} \omega_{\ell m}=m \Omega_{+}+\frac{\bar{\kappa} \epsilon}{2}\left[-i\left(N+\frac{1}{2}\right)-m \bar{\Omega} \pm \sqrt{{ }_{s} \bar{\lambda}_{\ell m}+m^{2} \bar{\Omega}^{2}-s(s+1)-\frac{1}{4}}\right]+\mathcal{O}\left(\epsilon^{2}\right),
$$

where $N=0,1,2, \ldots$ We remind the reader that $\Omega_{+}, \bar{\Omega}, \bar{\kappa}$ and ${ }_{s} \bar{\lambda}_{\ell m}$ are given in, respectively, eqs. (2.4), (3.76), (3.73) and (3.96). To the best of our knowledge, this result

\footnotetext{
${ }^{13}$ Even though eq. (3.87) is only valid for $s=0$, we assume that the condition $N=0,1,2, \ldots$ also holds for the other spins.
} 
is new in the literature. We emphasize that, even though we write eq. (4.16) as a small$\epsilon$ expansion, $\Omega_{+}$in it is exact and so not expanded for small $\epsilon$ (the reason being that $\Omega_{+}$became buried within $\bar{\omega}$ in eq. (3.92), which we kept fixed when carrying out the expansions).

We remind the reader that, up to this point, all of our expressions in this subsection were considering the limit $r \rightarrow r_{+}$(together with the small- $\epsilon$ expansion). By doing this, we obtained the modes that approach $m \Omega_{+}$in eq. (4.16). In order to obtain the modes that, instead, approach $m \Omega_{C}$, all we have to do is, in the quantities in eq. (4.16), swap $r_{+} \leftrightarrow r_{C}$ (which, in particular, implies $\epsilon \rightarrow-\epsilon$ ) and take the complex conjugate. Under this transformation, we obtain:

$$
{ }_{s} \omega_{\ell m}=m \Omega_{C}+\frac{\bar{\kappa} \epsilon}{2}\left[-i\left(N+\frac{1}{2}\right)+m \bar{\Omega} \pm \sqrt{{ }_{s} \bar{\lambda}_{\ell m}+m^{2} \bar{\Omega}^{2}-s(s+1)-\frac{1}{4}}\right]+\mathcal{O}\left(\epsilon^{2}\right)
$$

Eqs. (4.16) and (4.17) show that, as advanced in section 3.4, both the lower and the upper superradiant bound limits (i.e., $m \Omega_{C}$ and $m \Omega_{C}$, respectively) are accumulation points of QNMs as the extremal limit $r_{C} \rightarrow r_{+}$is approached. In order to obtain the next order correction $\bar{\omega}_{1}$, we need to expand all quantities in eqs. (4.10), (4.11) and (4.13) to one order higher in $\epsilon$. We then obtain:

$$
\begin{aligned}
\bar{\omega}_{1}= & \frac{L \bar{\kappa}\left[\left(\bar{r}_{+}-\bar{r}_{-}\right)\left(\bar{r}_{-}+3 \bar{r}_{+}\right)\right]^{-1}}{4\left(2 \bar{\omega}_{0} / \bar{\kappa}+m \bar{\Omega}+i(N+1 / 2)\right)}\left[i m \bar{\Omega}(2 N+1) \bar{r}_{+}\left(2-L \chi^{2} \bar{\kappa}\right)+\left.L \frac{d}{d \epsilon}{ }_{s} \lambda_{\ell m}\right|_{\epsilon=0}+\right. \\
& \left.+\frac{m \bar{\Omega}\left(\bar{r}_{+}+\bar{r}_{-}\right)^{2}\left(2 s^{2}+s-{ }_{s} \bar{\lambda}_{\ell m}\right)\left(m \bar{\Omega}+2 \bar{\omega}_{0} / \bar{\kappa}\right)}{2 \bar{r}_{+}\left({ }_{s} \bar{\lambda}_{\ell m}+m^{2} \bar{\Omega}^{2}-s^{2}-s\right)}\right]
\end{aligned}
$$

The $\epsilon$-derivative of ${ }_{s} \lambda_{\ell m}$ at $\epsilon=0$ can be written as

$$
\left.\frac{d}{d \epsilon}{ }_{s} \lambda_{\ell m}\right|_{\epsilon=0}=\bar{\kappa}\left(m \bar{\Omega}+2 \bar{\omega}_{0} / \bar{\kappa}\right) \frac{1}{2} \frac{d}{d \omega}{ }_{s} \lambda_{\ell m}\left(\omega=\frac{m a}{a^{2}+\bar{r}_{+}^{2}}\right) .
$$

To the best of our knowledge, no orders higher than leading-order in eq. (4.12) have been previously obtained in the literature for any spin. Above we have given explicit expressions for general $a$ for the two leading-order terms in eq. (4.12). Although one could, in principle, obtain higher order terms, we found it difficult to obtain them if keeping $\alpha=a / L$ general. On the other hand, by expanding the various quantities for small $\alpha$, we managed to reach up to one higher order in $\epsilon$. Specifically, what we did is the following. We obtained both eqs. (4.7) and (4.13) up to including $\mathcal{O}\left(\epsilon^{3}\right)$. We then expanded the coefficient of each $\epsilon$-order (including the $\bar{\omega}_{0}, \bar{\omega}_{1}, \ldots$ which appear within these coefficients, as well as the eigenvalue as given by (1.3) with eqs. (E.1)-(E.4)) for small $\alpha$ up to including $\mathcal{O}\left(\alpha^{3}\right)$. We then equated the left- and right-hand-sides at each order in this double series in $\alpha$ and $\epsilon$, thus obtaining an expansion for the QNM frequencies ${ }_{s} \omega_{\ell m}$ valid up to order $\left(\epsilon^{3}, \alpha^{3}\right)$. This expansion is the one in eq. (1.1), where the explicit form of the coefficients is given in appendix D. 


\section{Comparison with numerical calculation}

We have verified our expansions of the QNM frequencies and the angular eigenvalues with a numerical calculation. In this section, we first briefly sketch the approach used - we note that the method will be described in detail in [68]. We then present the numerical results as a check of the previous analyical expansions: eq. (1.1) (with the coefficients given in appendix D) for the QNM frequencies and eq. (1.3) for the angular eigenvalues.

The method we used for the numerical calculation is a direct implementation of the one described in [21], which, in its turn, is a generalization to Kerr-de Sitter of the technique introduced by Leaver in Kerr spacetime [23, 24]. Essentially, the method consists of expressing a solution of the radial ODE (3.1b) as a powers series in " $\left(r_{C}-r_{--}\right)\left(r-r_{+}\right) /((r-$ $\left.\left.r_{--}\right)\left(r_{C}-r_{+}\right)\right)$". By construction, this series satisfies the QNM boundary condition at the event horizon (which is the same as the ingoing boundary condition (3.34) there). However, this infinite series only satisfies the QNM boundary condition at the cosmological horizon (which is the same as the upgoing boundary condition (3.44) there) if it converges at that horizon. This convergence condition leads to an equation in terms of a 'radial' continued fraction which a frequency must satisfy for it to be a QNM frequency.

The method for calculating the angular eigenvalue is similar. One essentially expands a solution of the angular ODE (3.1a) about $\theta=\pi$, thus obeying the regularity condition there by construction. The regularity condition at the other endpoint, $\theta=0$, is only obeyed if ${ }_{s} \lambda_{\ell m}$ satisfies a certain equation in terms of an 'angular' continued fraction.

We numerically solve simultaneously for both the 'radial' continued fraction equation and the 'angular' continued fraction equation. The solution of this system of equations is then a QNM frequency $\omega={ }_{s} \omega_{\ell m}$ and an angular eigenvalue ${ }_{s} \lambda_{\ell m}$.

In figures 5 and 6 we plot token results of our numerical check. The plots show that each order in our small- $\epsilon$ expansion of the QNM frequencies consistently improves the expansion relative to the numerical results. The plots are for $s=-2$, but we also carried out numerical checks for $s=0,-1 / 2$ and -1 and found similarly good agreement with our analytical expansions.

\section{Conclusions}

Quasinormal Modes are an interesting topic of research from theoretical as well as observational points of view. In this work, we provided a new method for calculating QNMs frequencies and angular eigenvalues for Kerr-de Sitter black holes. This method consists of using the accessory parameter expansion, given in terms of monodromies of the associated Fuchsian ODE. In particular, we have extended the APE described in ref. [16] to the case where the monodromies are expanded in a small parameter $\epsilon$. This allowed us to obtain analytical expansions for both the angular eigenvalues and the QNM frequencies. Our expansion for the eigenvalues agrees with results in the literature, whereas, to the best of our knowledge, our high-order expansion for the frequencies is new. Finally, we provided numerical evidence that these analytical formulas are a very good approximation for reasonably small $\epsilon$. 

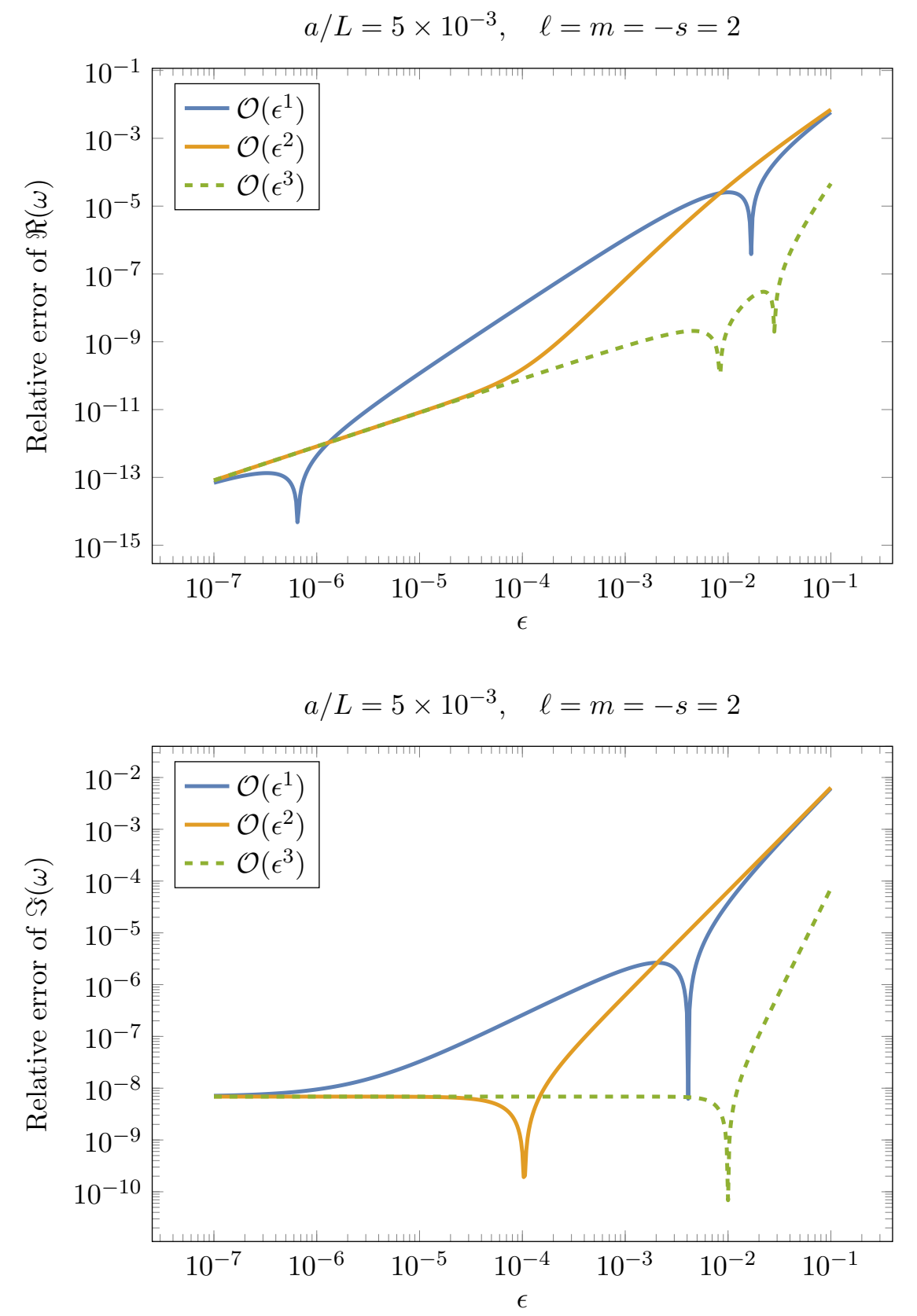

Figure 5. Relative error in the QNM frequencies when comparing our analytic expansion in eq. (1.1) with a numerical calculation. It is plotted as a function of $\epsilon$ and for the mode $s=-2$, $\ell=m=2$ and $a=5 \cdot 10^{-3} \mathrm{~L}$. The top graph is for the real part of the QNM frequency and the bottom graph for the imaginary part. Within each plot, the curve which is at the top corresponds to the relative error with the analytic expansion truncated to $O(\epsilon)$. The subsequent two lower curves correspond to truncation at $O\left(\epsilon^{2}\right)$ and $O\left(\epsilon^{3}\right)$, respectively. 

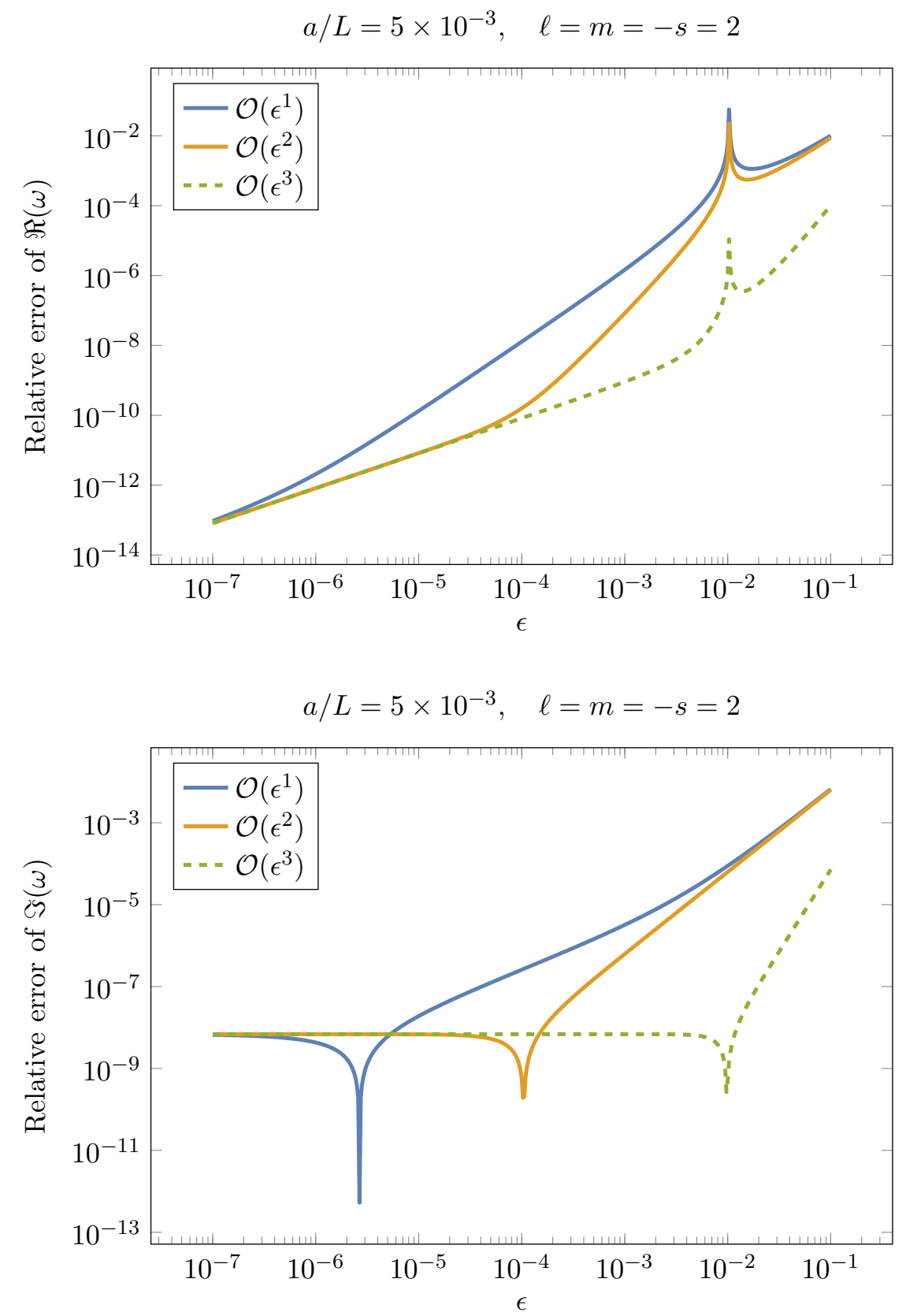

Figure 6. Similar to figure 5 but here it refers to the QNM frequency expansion about $m \Omega_{C}$ instead of $m \Omega_{+}$(i.e., the right hand side of eq. (1.1) after taking $\Omega_{+} \rightarrow \Omega_{C}, \epsilon \rightarrow-\epsilon$ and complex conjugating). 
There are a couple of directions for improving or extending our results. Firstly, as the $\tau$-function used here has a complete expansion, there is a possibility that a deeper study of its properties and symmetries can provide even more compact formulas or with faster convergence than the ones used here. Our implementation of the algorithm provided in [16] is limited as the expressions of order higher than $\epsilon^{3}$ are too cumbersome to be simplified with our available computing power. We are confident that simplification of these expressions is possible, but we leave this optimization for future work. Another possibility is to implement the APE numerically, as it was done in [17]. There are also other approaches to obtain the APE, like the ones in $[18,19,34,69]$ or the direct application of CFT operator product expansions, as a variation of what was done in $[15,16]$. Secondly, although our method works very well for a large region of configuration space $(\epsilon, a)$, we were limited to study the APE expansion close to the extremal limit $\epsilon \rightarrow 0$. We need the other extremal limit $\epsilon \rightarrow 1\left(r_{+} \rightarrow r_{-}\right)$in order to try to cover the full configuration space for Kerr-de Sitter black holes. This could in principle be implemented with an expansion of the $\tau$-function as $t \rightarrow 1$, but, in this case, the expansion would be in terms of the other composite monodromy $\sigma_{1 x}$. One would therefore need to study the relationship between $\sigma_{1 x}$ and $\sigma_{0 x}[15,70]$, which sets the relevant boundary conditions on the modes.

There are also other possible generalizations and applications of the APE method itself. For example, in [71], the scalar-field perturbation equations of a 5-dimensional Kerr-anti-de Sitter black hole were written as Heun equations and the eigenvalue and QNM conditions were written in terms of the monodromies (similarly to section 3.4 here in the case of Kerrde Sitter). The APE method applied to this black hole setting, verified numerically, is available in [72]. Moreover, the connection between the AdS/CFT approach to QNMs and the CFT structure behind isomonodromic method still remains to be completely understood.

One could also try to extend the APE to the case of an ODE with either less or more singular points. In particular, the APE could be used to study confluent cases of the Heun equation, corresponding to two regular and one irregular singular points. The linear perturbations of Kerr and Schwarzschild black holes are described by confluent Heun equations and the monodromy setup for these cases has been studied in [37]. However, this example still lacks a systematic expansion using the Painlevé $\mathrm{V} \tau$-function [73, 74], like in [16] for the Painlevé VI case. The same principle of finding the APE could also be used for an ODE possessing more than four singular points, using the respective $\tau$-function [75, 76]. It is known that linear perturbation equations for Kerr-NUT-(Anti-)de Sitter black holes are also reducible to separable ODEs for dimensions higher than four [77]. In particular, the number of singular points of these equations increases as the dimension increases.

Another potentially interesting, but speculative, line of investigation is to try to relate the isomonodromic approach with the Kerr/CFT correspondence [78], which so far only deals with the (near-)extremal $\left(r_{+}=r_{-}\right)$case [79]. The near-extremality expansion used here might be helpful to understand whether the CFT picture in the extremal case could in some sense be deformed to cater for the non-extremal case. In this sense, it is curious that the $\tau$-function can be understood as a $c=1 \mathrm{CFT}$ chiral correlator (even in the non-extremal case). However, the connection with the Kerr/CFT description, if any, still remains to be understood. Discussions along these lines can be found in $[28,71,80]$. In 
particular, there is also room to explore the Kerr-dS/CFT correspondence [62], which has been less explored than Kerr/CFT. The approach of [81] to obtain the full solutions of Heun's equation from CFT is also an interesting related direction.

Finally, the APE can also be used outside the realm of black hole physics. Particular examples are the Rabi model in quantum optics and its extensions $[82,83]$ and finding conformal mappings in 2-dimensions [17], as well as potential applications in condensed matter systems.

The APE method has opened new directions for studying spectral problems of Fuchsian ODEs - here, in particular, within the realm of black hole physics and Heun equations. We hope that the study presented here may be helpful for a better understanding of the integrable structure behind black holes, their QNMs and their connections to CFT.

\section{Acknowledgments}

We thank Alejandra Castro, Bruno Carneiro da Cunha and Amílcar Queiroz for stimulating discussions about this work. F.N. and M.L. acknowledge financial support from the Brazilian Ministry of Education (MEC). M.C. acknowledges partial financial support by CNPq (Brazil), process number 310200/2017-2.

\section{A Gauge transformations of Fuchsian equations}

Gauge transformations of Fuchsian equations are transformations that preserve the monodromy coefficients and the character of its singular points. ${ }^{14}$ There are two main gauge transformations in this sense: homographic transformations of the independent variable (also known as Möbius transformations) and s-homotopic transformations of the solution (i.e., multiplication by a solution of certain first order ODEs with polynomial coefficients) [36]. Here we apply these two transformations to reduce the master radial and angular equations (3.1) to its canonical and normal forms.

A faster route for obtaining the canonical form of eqs. (3.1a) and (3.1b) is to work directly with the corresponding linear system. Consider the following equation in selfadjoint form ${ }^{15}$

$$
\partial_{r}\left(U(r) \partial_{r} \psi(r)\right)-V(r) \psi(r)=0 .
$$

We consider the case that $U$ and $V$ are rational functions of $r$ and (A.1) has $n$ regular singular points at $r=r_{k}, k=1, \ldots, n$, with $r_{n}=\infty$. This equation can be rewritten as

$$
\left(\partial_{r}-A(r)\right) \Psi(r)=0, \quad A \equiv\left(\begin{array}{cc}
0 & U^{-1} \\
V & 0
\end{array}\right), \quad \Psi \equiv\left(\begin{array}{c}
\psi \\
U \partial_{r} \psi
\end{array}\right)
$$

As in electromagnetism, we shall call $A(r)$ the gauge connection of these Fuchsian equations. What happens to this system after performing a homographic transformation and a

\footnotetext{
${ }^{14}$ Except when these are apparent or removable singularities, which can be made explicit after a series of gauge transformations.

${ }^{15}$ We call the equation (A.1) self-adjoint even if its coefficients are complex.
} 
s-homotopic transformation? First, let us apply the following homographic transformation

$$
z \equiv \frac{d_{1} r+d_{2}}{d_{3} r+d_{4}} \quad \Rightarrow \quad \partial_{r}=F^{-1}(r) \partial_{z} \equiv \frac{d_{1} d_{4}-d_{2} d_{3}}{\left(d_{3} r+d_{4}\right)^{2}} \partial_{z}
$$

where $d_{1,2,3,4}$ are complex constants. This maps the points $r=r_{k}$ to new points $z=z_{k}$, $k=1, \ldots, n$, and leads to a new gauge connection

$$
\left(\partial_{z}-\tilde{A}(z)\right) \Psi(z)=0, \quad \tilde{A}=\left(\begin{array}{cc}
0 & \tilde{U}^{-1} \\
\tilde{V} & 0
\end{array}\right) \equiv\left(\begin{array}{cc}
0 & F U^{-1} \\
F V & 0
\end{array}\right)
$$

with $\Psi=\left(\psi(z), \tilde{U}(z) \partial_{z} \psi(z)\right)^{T}$. Now, let us apply the following s-homotopic transformation to the solution

$$
\psi=G(z) \tilde{\psi}=\prod_{i=1}^{n}\left(z-z_{i}\right)^{-\rho_{i} / 2} \tilde{\psi}, \quad \Rightarrow \quad \partial_{z} \psi=G \partial_{z} \tilde{\psi}+\left(\partial_{z} G\right) \tilde{\psi}=G\left(\partial_{z}+B\right) \tilde{\psi},
$$

for some $\rho_{i} \in \mathbb{C}$, where

$$
B \equiv-\sum_{i=1}^{n} \frac{\rho_{i} / 2}{z-z_{i}}
$$

Thus,

$$
\begin{aligned}
\Psi & =\left(G \tilde{\psi}, \tilde{U} G\left(\partial_{z}+B\right) \tilde{\psi}\right)^{T}=\hat{U}(z) \tilde{\Psi} \\
\partial_{z} \Psi & =\hat{U} \partial_{z} \tilde{\Psi}+\partial_{z} \hat{U} \tilde{\Psi}
\end{aligned}
$$

where

$$
\hat{U}(z) \equiv\left(\begin{array}{cc}
G & 0 \\
\tilde{U} G B & G
\end{array}\right), \quad \tilde{\Psi} \equiv\left(\tilde{\psi}, \tilde{U} \partial_{z} \tilde{\psi}\right)^{T}
$$

and the new gauge potential is $\overline{\tilde{A}}=\hat{U}^{-1} \tilde{A} \hat{U}-\hat{U}^{-1} \partial_{z} \hat{U}$. Calculating all terms, we have that

$$
\overline{\tilde{A}}=\left(\begin{array}{cc}
0 & \tilde{U}^{-1} \\
\tilde{V}-\tilde{U}\left(B^{2}+\partial_{z} B\right)-\partial_{z} \tilde{U} B & -2 B
\end{array}\right) .
$$

This can be simplified further by writing the linear system in terms of $\left(\tilde{\psi}, \partial_{z} \tilde{\psi}\right)^{T}$, removing $\tilde{U}$ by making

$$
\tilde{\Psi}=\left(\begin{array}{cc}
1 & 0 \\
0 & \tilde{U}
\end{array}\right)\left(\tilde{\psi}, \partial_{z} \tilde{\psi}\right)^{T}
$$

Removing the tildes and primes in the new gauge connection and linear system solution (i.e., relabel $\overline{\tilde{A}}$ by $A, \tilde{\Psi}$ by $\Psi$ and $\tilde{\psi}$ by $\psi$ ), we are left with a system of the form

$$
\partial_{z} \Psi=A(z) \Psi, \quad A=\left(\begin{array}{cc}
0 & 1 \\
\tilde{V} \tilde{U}^{-1}-\left(B^{2}+\partial_{z} B-B \partial_{z} \log \tilde{U}\right) & -2 B-\partial_{z} \log \tilde{U}
\end{array}\right),
$$

where $\Psi=\left(\psi, \partial_{z} \psi\right)^{T}$. This results in the following ODE for the transformed $\psi$

$$
\begin{gathered}
\partial_{z}^{2} \psi+P(z) \partial_{z} \psi+Q(z) \psi=0 \\
P(z) \equiv \partial_{z} \log \tilde{U}+2 B, \quad Q(z) \equiv B^{2}+\partial_{z} B+B \partial_{z} \log \tilde{U}-\tilde{V} \tilde{U}^{-1} .
\end{gathered}
$$




\section{A.1 Radial master equation}

The master radial equation $(3.1 \mathrm{~b})$ is of the form

$$
\partial_{r}\left(U(r) \partial_{r} R_{s, \omega, m}(r)\right)-V(r) R_{s, \omega, m}(r)=0,
$$

with

$$
\begin{aligned}
& U=\Delta_{r}^{s+1} \\
& V=U\left[-\left(\frac{W}{\Delta_{r}}\right)^{2}-i s\left(2 \frac{W^{\prime}}{\Delta_{r}}-\frac{W \Delta_{r}^{\prime}}{\Delta_{r}^{2}}\right)+\frac{Y_{s}}{\Delta_{r}}\right]
\end{aligned}
$$

We now apply the Möbius transformation

$$
z=\zeta_{\infty} \frac{\left(r-r_{1}\right)}{\left(r-r_{4}\right)} \equiv \frac{\left(r_{2}-r_{4}\right)}{\left(r_{2}-r_{1}\right)} \frac{\left(r-r_{1}\right)}{\left(r-r_{4}\right)}, \quad x \equiv \zeta_{\infty} \frac{r_{31}}{r_{34}}, \quad r_{i j} \equiv r_{i}-r_{j},
$$

with $i, j=1,2,3,4$, and the inverse transformation

$$
r=\frac{r_{4} z-r_{1} \zeta_{\infty}}{z-\zeta_{\infty}} \quad \Rightarrow \quad r-r_{i}=\frac{r_{4 i} z+r_{i 1} \zeta_{\infty}}{z-\zeta_{\infty}}, \quad i=1, \ldots, 4 .
$$

Then

$$
\partial_{r}=\frac{r_{14} \zeta_{\infty}}{\left(r-r_{4}\right)^{2}} \partial_{z}=-\frac{\left(z-\zeta_{\infty}\right)^{2}}{r_{41} \zeta_{\infty}} \partial_{z} \quad \Rightarrow \quad F(z)=-\frac{r_{41} \zeta_{\infty}}{\left(z-\zeta_{\infty}\right)^{2}}
$$

and

$$
\Delta_{r}=-\Delta_{r}^{\prime}\left(r_{4}\right) F(z) \frac{f(z)}{\left(z-\zeta_{\infty}\right)^{2}}, \quad f(z) \equiv z(z-1)(z-x) .
$$

We also apply the homotopic transformation

$$
R_{s, \omega, m}(r)=G(z) \psi(z), \quad G(z)=\left(z-\zeta_{\infty}\right)^{\beta} \prod_{i=1}^{3}\left(z-z_{i}\right)^{-\rho_{i} / 2},
$$

for some constant $\beta$, which generates a new ODE of the form (A.7). Notice that we omitted the indices $s, \omega, m$ in the solution, as they do not play any role in this appendix. To make (A.7) more explicit, first, we have

$$
\tilde{\Delta}_{r} \equiv F^{-1} \Delta_{r} \quad \Rightarrow \quad \tilde{U}=F^{-1} \Delta_{r}^{s+1}=F^{s} \tilde{\Delta}_{r}^{s+1}
$$

and

$$
\begin{aligned}
\frac{\tilde{V}}{\tilde{U}} & =F^{2} \frac{V}{U} \\
& =\left[-\left(\frac{W}{\tilde{\Delta}_{r}}\right)^{2}-i s\left(2 \frac{\partial_{z} W}{\tilde{\Delta}_{r}}-\frac{W \partial_{z}\left(F \tilde{\Delta}_{r}\right)}{F \tilde{\Delta}_{r}^{2}}\right)+F \frac{Y_{s}}{\tilde{\Delta}_{r}}\right] \\
& =\left[-\left(\frac{W}{\tilde{\Delta}_{r}}\right)^{2}-i s\left(2 \partial_{z}\left(\frac{W}{\tilde{\Delta}_{r}}\right)+\frac{W}{\tilde{\Delta}_{r}} \partial_{z} \log \left(\frac{\tilde{\Delta}_{r}}{F}\right)\right)+F \frac{Y_{s}}{\tilde{\Delta}_{r}}\right] .
\end{aligned}
$$


Then, we can find that

$$
P(z)=\frac{2(\beta-(2 s+1))}{z-\zeta_{\infty}}+\sum_{i=1}^{3} \frac{1+s-\rho_{i}}{z-z_{i}}
$$

and

$$
Q(z)=-\frac{\tilde{V}}{\tilde{U}}+\frac{\beta^{2}-\beta(4 s+3)}{\left(z-\zeta_{\infty}\right)^{2}}+\frac{q_{\infty}}{z-\zeta_{\infty}}+\sum_{i=1}^{3}\left(\frac{\rho_{i}\left(\rho_{i}-2 s\right) / 4}{\left(z-z_{i}\right)^{2}}+\frac{q_{i}}{z-z_{i}}\right)
$$

with

$$
\begin{aligned}
q_{\infty} & =-\sum_{i=1}^{3} \frac{(1+s) \beta+(1+2 s-\beta) \rho_{i}}{z_{i}-\zeta_{\infty}} \\
q_{i} & =\frac{(1+s) \beta+(1+2 s-\beta) \rho_{i}}{z_{i}-\zeta_{\infty}}+\sum_{j=1, j \neq i}^{3} \frac{\rho_{i} \rho_{j}-(1+s)\left(\rho_{i}+\rho_{j}\right)}{2\left(z_{i}-z_{j}\right)}, \quad i=1, \ldots, 3 .
\end{aligned}
$$

It is helpful to expand eq. (A.16) into partial fractions. Essentially, we have the following two types of terms:

$$
\frac{A r^{2}+B}{\tilde{\Delta}_{r}}, \quad\left(\frac{C r^{2}+D}{\tilde{\Delta}_{r}}\right) F(z),
$$

for some constants $A, B, C, D$. The main difference between these two terms is that the first one has no pole at $z=\zeta_{\infty}$, whereas the second one has a pole there due to the $F(z)$ factor. Thus, we have

$$
\frac{W}{\tilde{\Delta}_{r}}=\sum_{k=0,1, x} \frac{W_{k}}{z-z_{k}}, \quad W_{k} \equiv \operatorname{res}_{r=r_{k}} \frac{W(r)}{\Delta_{r}(r)}=\frac{\chi^{2}\left(\omega\left(r_{k}^{2}+a^{2}\right)-a m\right)}{\Delta_{r}^{\prime}\left(r_{k}\right)},
$$

and

$$
F \frac{Y_{s}}{\tilde{\Delta}_{r}}=\frac{2(s+1)(2 s+1)\left(\sum_{i=1}^{3} r_{i}-r_{4}\right) /\left(r_{14} \zeta_{\infty}\right)}{z-\zeta_{\infty}}-\frac{2(s+1)(2 s+1)}{\left(z-\zeta_{\infty}\right)^{2}}+\sum_{i=0,1, x} F\left(z_{i}\right) \frac{Y_{s, i}}{z-z_{i}},
$$

with

$$
Y_{s, i} \equiv \frac{2(s+1)(2 s+1) r_{i}^{2} / L^{2}-s\left(1-a^{2} / L^{2}\right)+{ }_{s} \lambda_{\ell m}}{\Delta_{r}^{\prime}\left(r_{i}\right)} .
$$

Then, going back to eq. (A.16), we get

$$
\begin{aligned}
-\frac{\tilde{V}}{\tilde{U}}= & \frac{2(s+1)(2 s+1)}{\left(z-\zeta_{\infty}\right)^{2}}-\frac{2(s+1)(2 s+1)\left(\sum_{i=1}^{3} r_{i}-r_{4}\right) /\left(r_{14} \zeta_{\infty}\right)}{z-\zeta_{\infty}}+ \\
& +\sum_{k=1}^{3}\left(\frac{W_{k}\left(W_{k}-i s\right)}{\left(z-z_{k}\right)^{2}}+\frac{v_{k}}{z-z_{k}}\right)
\end{aligned}
$$

with

$$
v_{k} \equiv-F\left(z_{k}\right) Y_{s, k}+\sum_{j=1, j \neq k}^{3} \frac{2 W_{k} W_{j}+i s\left(W_{k}+W_{j}\right)}{z_{k}-z_{j}}, \quad k=1,2,3 .
$$


Let us first check that the singularity $z=\zeta_{\infty}$ is removable. We start by demanding that

$$
\begin{aligned}
\operatorname{res}_{z=z_{k}} P(z) & =2(\beta-(2 s+1))=0, \\
\operatorname{res}_{z=z_{k}}\left(z-z_{k}\right) Q(z) & =\beta^{2}-(4 s+3) \beta+(2 s+1)(2 s+2)=0 .
\end{aligned}
$$

Both equations have a solution for $\beta=2 s+1$ and we will assume this value for $\beta$ from now on in this subsection. Now, quite surprisingly, substituting $\beta$ in eq. (A.18), we obtain

$$
\begin{aligned}
\operatorname{res}_{z=z_{k}} Q(z) & =-\frac{(2 s+2)(2 s+1)\left(\sum_{i=1}^{3} r_{i}-r_{4}\right)}{r_{14} \zeta_{\infty}}-\sum_{i=1}^{3} \frac{(1+s)(2 s+1)}{z_{i \infty}} \\
& =\frac{(2 s+1)(2 s+2)}{r_{14} \zeta_{\infty}}\left(r_{4}+\sum_{i=1}^{3} \frac{\left(r_{i}-r_{4}\right)}{2}-\sum_{i=1}^{3} r_{i}\right) \\
& =-\frac{(2 s+1)(2 s+2)}{2 r_{14} \zeta_{\infty}}\left(r_{4}+\sum_{i=1}^{3} r_{i}\right)=0,
\end{aligned}
$$

where in the second line we used $z_{i \infty}=-r_{14} \zeta_{\infty} / r_{i 4}$, coming from eq. (A.12), and in the last line we used the fact that there is no third order term in the polynomial $\Delta_{r}(r)$. Therefore, the singularity $z=\zeta_{\infty}$ is completely removable from the ODE. This fact has been proved for any type $\mathrm{D}$ vacuum solution with cosmological constant in [10]. Our derivation is an improvement to that work, as it gives the explicit dependence on the $\rho_{i}$ and a more compact and suggestive final result in terms of the monodromies.

At this stage, we have

$$
\begin{aligned}
P(z) & =\sum_{k=1}^{3} \frac{1+s-\rho_{k}}{z-z_{k}}, \\
Q(z) & =\sum_{k=1}^{3}\left(\frac{\rho_{k}\left(\rho_{k}-2 s\right) / 4+W_{k}\left(W_{k}-i s\right)}{\left(z-z_{k}\right)^{2}}+\frac{q_{k}+v_{k}}{z-z_{k}}\right), \\
q_{k} & =\frac{(1+s)(1+2 s)}{z_{k}-\zeta_{\infty}}+\sum_{j=1, j \neq k}^{3} \frac{\rho_{k} \rho_{j}-(1+s)\left(\rho_{k}+\rho_{j}\right)}{2\left(z_{k}-z_{j}\right)}, \\
v_{k} & =\frac{r_{41} \zeta_{\infty}}{\left(z_{k}-\zeta_{\infty}\right)^{2}} Y_{s, k}+\sum_{j=1, j \neq k}^{3} \frac{2 W_{k} W_{j}+i s\left(W_{k}+W_{j}\right)}{z_{k}-z_{j}}, \quad k=1,2,3 .
\end{aligned}
$$

We now have two possibilities to simplify the Heun equation (A.7): the canonical form and the normal form.

Canonical form. In order to find the canonical form of the Heun equation, we choose $\rho_{k}$ so as to cancel the $\left(z-z_{k}\right)^{-2}$ term in $Q(z)$ in (A.18). In order to find the Frobenius exponents $\rho_{k}$, we take

$$
\operatorname{res}_{z=z_{k}}\left(z-z_{k}\right) Q(z)=\frac{1}{4} \rho_{k}\left(\rho_{k}-2 s\right)+W_{k}\left(W_{k}-i s\right)=0 .
$$

Its roots are given by

$$
\rho_{k}^{(-)}=-2 i W_{k}, \quad \rho_{k}^{(+)}=2 i W_{k}+2 s \quad \Rightarrow \quad \rho_{k}^{( \pm)}=s \pm\left(2 i W_{k}+s\right), \quad k=1,2,3 .
$$


Then, if we define $\theta_{k}=i W_{k}+s / 2$ and choose $\rho_{k}=\rho_{k}^{(+)}=s+2 \theta_{k}$ we obtain

$$
\begin{aligned}
P(z) & =\sum_{k=1}^{3} \frac{1-2 \theta_{k}}{z-z_{k}}, \quad Q(z)=\sum_{k=1}^{3} \frac{Q_{k}}{z-z_{k}}, \\
Q_{k} & =\frac{(1+s)(1+2 s)}{z_{k}-\zeta_{\infty}}-F\left(z_{k}\right) Y_{s, k}+\sum_{j=1, j \neq k}^{3} \frac{(2 s-1)\left(\theta_{k}+\theta_{j}-s\right)-2 s}{z_{k}-z_{j}} .
\end{aligned}
$$

We can rewrite the last term in two convenient ways

$$
\begin{aligned}
-F\left(z_{k}\right) Y_{s, k} & =-\frac{L^{2}}{z_{k}-\zeta_{\infty}} \frac{\left(2(2 s+1)(s+1) r_{k}^{2} / L^{2}+{ }_{s} \lambda_{\ell m}-s\left(1-a^{2} / L^{2}\right)\right)}{\prod_{j=1, j \neq k}^{3}\left(r_{k}-r_{j}\right)} \\
& =\frac{\left(r_{1}-r_{4}\right) \zeta_{\infty}}{\Delta_{r}^{\prime}\left(r_{4}\right)} \frac{2(2 s+1)(s+1) r_{k}^{2} / L^{2}+{ }_{s} \lambda_{\ell m}-s\left(1-a^{2} / L^{2}\right)}{f^{\prime}\left(z_{k}\right)} \\
& =-\frac{L^{2}}{\left(r_{1}-r_{2}\right)\left(r_{3}-r_{4}\right)} \frac{\left(2(2 s+1)(s+1) r_{k}^{2} / L^{2}+{ }_{s} \lambda_{\ell m}-s\left(1-a^{2} / L^{2}\right)\right)}{f^{\prime}\left(z_{k}\right)}
\end{aligned}
$$

Comparing with the radial Heun equation (3.17), we can extract Heun's accessory parameter in (A.35) by $K_{x}=-\operatorname{res}_{z=x} Q(z)=-Q_{3}$ and this gives

$$
\begin{aligned}
K_{x}= & \frac{(1-2 s)\left(\theta_{x}+\theta_{0}-s\right)+2 s}{x}+\frac{(1-2 s)\left(\theta_{x}+\theta_{1}-s\right)+2 s}{x-1}- \\
& -\frac{1}{x-\zeta_{\infty}}\left[(1+s)(1+2 s)-\frac{\left(2(2 s+1)(s+1) r_{k}^{2}+{ }_{s} \lambda_{\ell m} L^{2}-s L^{2}\left(1-a^{2} / L^{2}\right)\right)}{\left(r_{3}-r_{1}\right)\left(r_{3}-r_{2}\right)}\right]
\end{aligned}
$$

or

$$
\begin{aligned}
K_{x}= & \frac{(1-2 s)\left(\theta_{x}+\theta_{0}-s\right)+2 s}{x}+\frac{(1-2 s)\left(\theta_{x}+\theta_{1}-s\right)+2 s}{x-1}- \\
& -\frac{(1+s)(1+2 s)}{x-\zeta_{\infty}}+\frac{1}{\left(r_{1}-r_{2}\right)\left(r_{3}-r_{4}\right)} \frac{\left(2(2 s+1)(s+1) r_{k}^{2}+{ }_{s} \lambda_{\ell m} L^{2}-s L^{2}\left(1-a^{2} / L^{2}\right)\right)}{x(x-1)}
\end{aligned}
$$

which is the best form to multiply it by $x(x-1)$.

Normal form. In the APE, we actually use the normal form of the accessory parameter, since our formulas for the $\tau$-function are adapted to this form. The normal form is obtained by making $\rho_{k}=1+s, k=1,2,3$ in eq. (A.32), which yields

$$
\begin{aligned}
P(z) & =0, \quad Q(z)=\sum_{k=1}^{3}\left(\frac{\frac{1}{4}-\theta_{k}^{2}}{\left(z-z_{k}\right)^{2}}+\frac{\tilde{Q}_{k}}{z-z_{k}}\right), \\
\tilde{Q}_{k} & =\frac{(1+s)(1+2 s)}{z_{k}-\zeta_{\infty}}-F\left(z_{k}\right) Y_{s, k}+\sum_{j=1, j \neq k}^{3} \frac{-\frac{1}{2}\left(1+2 s+4 \theta_{j} \theta_{k}\right)+2 s\left(\theta_{j}+\theta_{k}-s\right)}{z_{k}-z_{j}} .
\end{aligned}
$$

Then, $\mathcal{H}_{x}=-\operatorname{res}_{z=x} Q(z)=-\tilde{Q}_{3}$, which is related to (A.40) by (4.6). 


\section{A.2 Angular master equation}

Most of the expressions in this angular subsection readily follow from those in the previous radial case presented in subsection A.1. Since they are separate subsections, we shall use some of the same symbols to denote quantities here defined as the quantities with the same symbol in section A.1 merely under the change $r \rightarrow u$, and so we will not give the definitions of those symbols explicitly here.

The angular master equation (3.1a) is of the form

$$
\partial_{u}\left(U(u) \partial_{u} S(u)\right)-V(u) S(u)=0,
$$

with

$$
\begin{aligned}
& U=\Delta_{u}, \\
& V=-U\left[-\left(\frac{H+\frac{s}{2} \Delta_{u}^{\prime}}{\Delta_{u}}\right)^{2}+2 s \frac{H^{\prime}}{\Delta_{u}}-\frac{X_{s}}{\Delta_{u}}\right] .
\end{aligned}
$$

Applying similar transformations as in section A.1, we find

$$
\begin{aligned}
P(z) & =\frac{2(\beta-1)}{z-\zeta_{\infty}}+\sum_{k=1}^{3} \frac{1-\rho_{k}}{z-z_{k}} \\
Q(z) & =-\frac{\tilde{V}}{\tilde{U}}+\frac{\beta^{2}-3 \beta}{\left(z-\zeta_{\infty}\right)^{2}}+\frac{q_{\infty}}{z-\zeta_{\infty}}+\sum_{k=1}^{3}\left(\frac{\rho_{k}^{2} / 4}{\left(z-z_{k}\right)^{2}}+\frac{q_{k}}{z-z_{k}}\right) \\
q_{k} & =\frac{\beta+(1-\beta) \rho_{k}}{z_{k}-\zeta_{\infty}}+\sum_{j=1, j \neq k}^{3} \frac{\rho_{k} \rho_{j}-\left(\rho_{k}+\rho_{j}\right)}{2\left(z_{k}-z_{j}\right)} \\
q_{\infty} & =-\sum_{k=1}^{3} \frac{\beta+(1-\beta) \rho_{k}}{z_{k}-\zeta_{\infty}} .
\end{aligned}
$$

We also have

$$
\begin{aligned}
-\frac{\tilde{V}}{\tilde{U}} & =-\left[\frac{H}{\tilde{\Delta}_{u}}+\frac{s}{2} \partial_{z} \log \left(F \tilde{\Delta}_{u}\right)\right]^{2}+2 s\left[\partial_{z}\left(\frac{H}{\tilde{\Delta}_{u}}\right)+\frac{H}{\tilde{\Delta}_{u}} \partial_{z} \log \tilde{\Delta}_{u}\right]-F \frac{X_{s}}{\tilde{\Delta}_{u}} \\
& =\frac{2}{\left(z-\zeta_{\infty}\right)^{2}}+\frac{v_{\infty}}{z-\zeta_{\infty}}+\sum_{k=1}^{3}\left(\frac{-\left(H_{k}+\frac{s}{2}\right)^{2}}{\left(z-z_{k}\right)^{2}}+\frac{v_{k}}{z-z_{k}}\right) \\
v_{k} & =\frac{2 s^{2}}{z_{k}-\zeta_{\infty}}-F\left(z_{k}\right) X_{s, k}-\sum_{j=1, j \neq k}^{3} \frac{2\left(H_{k}-s / 2\right)\left(H_{j}-s / 2\right)}{z_{k}-z_{j}} \\
v_{\infty} & =-\sum_{k=1}^{3} \frac{2 s^{2}}{z_{k}-\zeta_{\infty}}-\frac{2\left(2 s^{2}+1\right)\left(-u_{4}+\sum_{j=1}^{3} u_{j}\right)}{u_{14} \zeta_{\infty}} \\
X_{s, k} & =\frac{2\left(2 s^{2}+1\right) \alpha^{2} u_{k}^{2}-{ }_{s} \lambda_{\ell m}}{\Delta_{u}^{\prime}\left(u_{k}\right)}
\end{aligned}
$$


with $k=1,2,3$, where we used that

$$
\frac{H}{\tilde{\Delta}_{u}}=\sum_{k=1}^{3} \frac{H_{k}}{z-z_{k}}, \quad \tilde{\Delta}_{u}=-\Delta_{u}^{\prime}\left(u_{4}\right) \frac{f(z)}{\left(z-\zeta_{\infty}\right)^{2}}, \quad F\left(z_{k}\right)=\frac{u_{41} \zeta_{\infty}}{\left(z_{k}-\zeta_{\infty}\right)^{2}} .
$$

Using eq. (A.50a) in eq. (A.46), we find

$$
Q(z)=\sum_{k=1}^{3}\left(\frac{\rho_{k}^{2} / 4-\left(H_{k}+s / 2\right)^{2}}{\left(z-z_{k}\right)^{2}}+\frac{q_{k}+v_{k}}{z-z_{k}}\right)+\frac{\beta^{2}-3 \beta+2}{\left(z-\zeta_{\infty}\right)^{2}}+\frac{q_{\infty}+v_{\infty}}{z-\zeta_{\infty}} .
$$

For $\beta=1$, the two terms with poles at $z=\zeta_{\infty}$ vanish identically, as in the radial case.

Canonical form. If we substitute $\rho_{k}=2 \theta_{k}=-2\left(H_{k}+s / 2\right)$ in (A.52), we find

$$
\begin{aligned}
P(z) & =\sum_{k=1}^{3} \frac{1-2 \theta_{k}}{z-z_{k}}, \quad Q(z)=\sum_{k=1}^{3} \frac{Q_{k}}{z-z_{k}}, \\
Q_{k} & =\frac{2 s^{2}+1}{z_{k}-\zeta_{\infty}}-\frac{1}{u_{12} u_{34}} \frac{2\left(2 s^{2}+1\right) u_{k}^{2}-{ }_{s} \lambda_{\ell m} \alpha^{-2}}{x(x-1)}+\sum_{j=1, j \neq k}^{3} \frac{-(2 s+1)\left(\theta_{k}+\theta_{j}+s\right)+s}{z_{k}-z_{j}},
\end{aligned}
$$

with $k=1,2,3$.

Normal form. Substituting $\rho_{k}=1$ in (A.52), we get

$$
\begin{aligned}
P(z) & =0, \quad Q(z)=\sum_{k=1}^{3}\left(\frac{\frac{1}{4}-\theta_{k}^{2}}{\left(z-z_{k}\right)^{2}}+\frac{\tilde{Q}_{k}}{z-z_{k}}\right), \\
\tilde{Q}_{k} & =\frac{2 s^{2}+1}{z_{k}-\zeta_{\infty}}-\frac{1}{u_{12} u_{34}} \frac{2\left(2 s^{2}+1\right) u_{k}^{2}-{ }_{s} \lambda_{\ell m} \alpha^{-2}}{x(x-1)}-\sum_{j=1, j \neq k}^{3} \frac{1+4\left(\theta_{k}-s\right)\left(\theta_{j}-s\right)}{2\left(z_{k}-z_{j}\right)} .
\end{aligned}
$$

\section{B Alternative derivation of quasinormal mode equation}

In this appendix, we show how to obtain eq. (4.15) using a path-multiplicative solution [12] to leading order in $x \rightarrow 0$. First, let us rewrite eq. (3.5) in Euler form

$$
\left\{(z-x) D_{\mathrm{HG}}\left[b_{1}, b_{2} ; 1-2 \theta_{0}\right]+\left(1-2 \theta_{x}\right) z D_{z}-x(x-1) K_{x} z\right\} y(z)=0,
$$

where $D_{z} \equiv z \partial_{z}$ is the Euler derivative and we define the hypergeometric differential operator as

$$
D_{H G}[A, B ; C] \equiv z\left(D_{z}+A\right)\left(D_{z}+B\right)-D_{z}\left(D_{z}+C-1\right),
$$

with $A, B, C$ arbitrary constants. The operator $D_{H G}$ is the operator in the Euler form of the hypergeometric equation, i.e., $D_{H G}[A, B ; C]_{2} \mathrm{~F}_{1}(A, B ; C ; z)=0$.

Now, let us assume that $y(z)=z^{\nu} h_{\nu}(z)+\mathcal{O}(x)$, with $\nu$ a constant. For $x=0$, the monodromy of this solution around $z=0$ must be equal to the composite monodromy of the full solution around $z=0$ and $z=x$ to leading order in $x$ [16]. This type of solution is 
called a path-multiplicative solution by [12]. If we plug the small- $x$ expansion of $y(z)$ into eq. (B.1), we obtain

$$
\left(D_{H G}\left[b_{1,0}+\nu, b_{2,0}+\nu ; 2\left(1-\theta_{0,0}-\theta_{x, 0}+\nu\right)\right]-\nu\left(\nu+1-2 \theta_{0,0}-2 \theta_{x, 0}\right)+\tilde{K}_{x}\right) h_{\nu}(z)=0
$$

where $\tilde{K}_{x} \equiv-\lim _{x \rightarrow 0} x(x-1) K_{x}$. Here, $\theta_{0,0}, \theta_{x, 0}$ and $b_{k, 0}$ denote the Frobenius exponents $\theta_{0}, \theta_{x}$ and $b_{k}$ of (B.1), respectively, calculated at $x=0$. Therefore, if we make the choice

$$
\nu\left(\nu+1-2 \theta_{0,0}-2 \theta_{x, 0}\right)-\tilde{K}_{x}=0,
$$

we have that $h_{\nu}(z)$ is a solution of the hypergeometric equation

$$
D_{H G}\left[b_{1,0}+\nu, b_{2,0}+\nu ; 2\left(1-\theta_{0,0}-\theta_{x, 0}+\nu\right)\right] h_{\nu}(z)=0 .
$$

Now, we use the two solutions of the quadratic equation (B.4) for $\nu$, which we denote by $\nu_{ \pm}$, to obtain the composite monodromy as $x \rightarrow 0$

$$
\sigma_{0}=\frac{1}{2}\left(\nu_{+}-\nu_{-}\right)=\frac{1}{2} \sqrt{\left(2 \theta_{0,0}+2 \theta_{x, 0}-1\right)^{2}+4 \tilde{K}_{x}}
$$

where $\sigma_{0} \equiv \lim _{x \rightarrow 0} \sigma_{0 x}$ (see eq. (4.1a)). Finally, by equating the QNM condition in eq. (3.62)

$$
\sigma_{0}= \pm\left(\theta_{0,0}-\theta_{x, 0}\right)+N+\frac{1}{2}
$$

and the appropriate monodromies (4.11) onto eq. (B.6), we obtain the correct eq. (4.14) and the corresponding QNMs. Finally, we see that, since $\nu_{ \pm}=\theta_{0,0}+\theta_{x, 0}-\frac{1}{2} \pm \sigma_{0}$, the monodromy matrix of the solution $z^{\nu} h_{\nu}(z)$ is given by

$$
M_{0 x}=\left(\begin{array}{cc}
e^{2 \pi i \nu_{+}} & 0 \\
0 & e^{2 \pi i \nu_{-}}
\end{array}\right) \sim\left(\begin{array}{cc}
-e^{2 \pi i \sigma_{0}} & 0 \\
0 & -e^{-2 \pi i \sigma_{0}}
\end{array}\right) .
$$

This is the origin of the minus sign in the composite trace definition in eq. (3.59).

\section{Accessory parameter expansion from isomonodromic $\tau$-function}

Here we adapt the approach of [16] for the APE to the case of monodromies that depend on $x$. The accessory parameter $K_{x}$ of the Heun equation can be obtained via the isomonodromic $\tau$-function by

$$
K_{x}=\left.\frac{d}{d t} \log \left(t^{-4 \theta_{0} \theta_{t}}(1-t)^{-4 \theta_{1} \theta_{t}} \tau\left(\boldsymbol{\theta}_{0,+} ; t\right)\right)\right|_{t=x, \lambda(x)=x}
$$

where

$$
\boldsymbol{\theta}_{s_{1}, s_{2}} \equiv\left(\theta_{0}, \theta_{1}, \theta_{t}+\frac{s_{1} 1}{2}, \theta_{\infty}+\frac{s_{2} 1}{2}\right), \quad s_{1}, s_{2}=0, \pm .
$$


The general definition of the $\tau$-function can be found in [16, 84], for example. The $\tau$ function satisfies a second-order non-linear ODE which is related to the Painlevé VI equation. This second-order ODE has two integration constants $(\sigma, \mathrm{s}) .{ }^{16}$ The integration constant $\sigma$ is in fact the composite monodromy of Heun solutions as discussed in this paper and $\mathrm{s}$ is related to the other composite monodromies in a precise way.

The usefulness of the approach via eq. (C.1) relies on the complete expansion of the $\tau$-function in terms of monodromy data, first obtained in [84]. Such expansion is

$$
\tau(\boldsymbol{\theta} ; t) \equiv t^{2 \theta_{0} \theta_{t}}(1-t)^{4 \theta_{1} \theta_{t}} t^{\sigma^{2}-\theta_{0}^{2}-\theta_{t}^{2}} \sum_{n \in \mathbb{Z}} C(\boldsymbol{\theta}, \sigma+n) \mathbf{s}^{n} t^{n(n+2 \sigma)} \mathcal{B}(\boldsymbol{\theta}, \sigma+n ; t),
$$

where the structure constants $C(\boldsymbol{\theta}, \sigma)$ are given in terms of Barnes functions $G$ as $^{17}$

$$
C(\boldsymbol{\theta}, \sigma)=\frac{\prod_{\varepsilon, \tilde{\varepsilon}= \pm} G\left(1+\theta_{t}+\varepsilon \theta_{0}+\tilde{\varepsilon} \sigma\right) G\left(1+\theta_{1}+\varepsilon \theta_{\infty}+\tilde{\varepsilon} \sigma\right)}{\prod_{\varepsilon= \pm} G(1+\varepsilon 2 \sigma)} .
$$

The $\mathcal{B}$ 's in eq. (C.3) are the conformal blocks for central charge $c=1$, given by the AldayGaiotto-Tachikawa (AGT) [85] combinatorial series

$$
\mathcal{B}(\boldsymbol{\theta}, \sigma ; t)=\sum_{n, m \in \mathbb{Y}} \mathcal{B}_{n, m}(\boldsymbol{\theta}, \sigma) t^{|n|+|m|},
$$

summing over pairs of Young diagrams $n, m$ with

$$
\begin{aligned}
\mathcal{B}_{n, m}(\boldsymbol{\theta}, \sigma)= & \prod_{(i, j) \in n} \frac{\left(\left(\theta_{t}+\sigma+i-j\right)^{2}-\theta_{0}^{2}\right)\left(\left(\theta_{1}+\sigma+i-j\right)^{2}-\theta_{\infty}^{2}\right)}{h_{n}^{2}(i, j)\left(n_{j}^{\prime}+m_{i}-i-j+1+2 \sigma\right)^{2}} \\
& \times \prod_{(i, j) \in m} \frac{\left(\left(\theta_{t}-\sigma+i-j\right)^{2}-\theta_{0}^{2}\right)\left(\left(\theta_{1}-\sigma+i-j\right)^{2}-\theta_{\infty}^{2}\right)}{h_{m}^{2}(i, j)\left(n_{i}+m_{j}^{\prime}-i-j+1-2 \sigma\right)^{2}}
\end{aligned}
$$

where $(i, j)$ denotes the respective box in a Young diagram $n, n_{i}$ the number of boxes in row $i, n_{j}^{\prime}$ the number of boxes in column $j$ and $h_{n}(i, j)=n_{i}+n_{j}^{\prime}-i-j+1$ its hook length.

As mentioned, the two integration constants of the $\tau$-function are $(\sigma, \mathrm{s})$ and, as shown in [16], the condition $\lambda(x)=x$ introduces a constraint $\mathrm{s}=\mathrm{s}(\boldsymbol{\theta}, \sigma, x)=\mathrm{s}_{0}+\mathrm{s}_{1} x+\mathcal{O}\left(x^{2}\right)$, with $\mathrm{s}_{i}=\mathrm{s}_{i}\left(\theta_{k}, \sigma\right)$. In order to find the APE, we just need to plug this constraint into eq. (C.1) and consistently expand the result for small $x$.

The main difference between the APE used in the present paper and the one in [16] is that here the monodromies depend on the moduli $x=x(\epsilon)$ via the parameter $\epsilon$. As explained in the main text, we assume the monodromies and moduli have regular expansions in $\epsilon$ given by (4.1). If we solve the condition $\lambda(x)=x$ for $s$ as an expansion in $\epsilon$ using the expanded monodromies and moduli, we get $\mathbf{s}=\tilde{\mathbf{s}}_{0}+\tilde{\mathbf{s}}_{1} \epsilon+\mathcal{O}\left(\epsilon^{2}\right)$, with $\tilde{\mathbf{s}}_{i}=\tilde{\mathbf{s}}_{i}\left(\theta_{k, j}, \sigma_{j}\right)$, and this result can be used in (C.1) to obtain our main result (4.7).

\footnotetext{
${ }^{16}$ Note that this integration constant is denoted by a sans-serif letter $\mathrm{s}$ in order to distinguish it from the spin $s$. Similarly, we use $\varepsilon$ in eq. (C.4) in order to distinguish it from the extremality parameter $\epsilon$ elsewhere in the paper.

${ }^{17}$ Barnes functions are defined by the functional relation $G(z+1)=\Gamma(z) G(z)$, with $G(1)=1$ and $\Gamma(z)$ being the Euler gamma function.
} 


\section{Coefficients of the quasinormal mode expansion}

In this appendix, we provide expressions below are the coefficients in eq. (1.1) expanded up to $\mathcal{O}\left(a^{3}\right)$. For simplicity, in this appendix we take $L=1$ and we define $\delta_{\sigma} \equiv \ell(\ell+1)$ and $\zeta \equiv \sqrt{12 \delta_{\sigma}-8 s^{2}+5}$.

General-spin expressions for the first two coefficients are the following:

$$
\begin{aligned}
& \bar{\omega}_{0}=\frac{1}{4} \sqrt{3}\left(1-14 a^{2}\right) \zeta+\frac{3}{4} i\left(7 a^{2}(2 N+1)+4 i \sqrt{3} a m-2 N-1\right)+\frac{\sqrt{3} a^{2}}{4 \zeta \delta_{\sigma}\left(4 \delta_{\sigma}-3\right)} \\
& \times\left\{516 \delta_{\sigma}^{3}+4 \delta_{\sigma}^{2}\left(45 m^{2}-74 s^{2}-46\right)+36 m^{2} s^{2}\left(s^{2}+2\right)-3 \delta_{\sigma}\left[m^{2}\left(40 s^{2}+44\right)+4 s^{4}-76 s^{2}+51\right]\right\} \\
& +\mathcal{O}\left(a^{4}\right), \\
& \bar{\omega}_{1}=-3 a m-\frac{9 a m s^{2}\left(\zeta-i \sqrt{3}(2 N+1)\left(\delta_{\sigma}-s^{2}+1\right)\right)}{2 \zeta \delta_{\sigma}\left(3 \delta_{\sigma}-2 s^{2}+2\right)} \\
& +\frac{9 i \sqrt{3} a^{3} m(2 N+1)}{2 \zeta^{3}\left(\delta_{\sigma}-2\right) \delta_{\sigma}^{3}\left(4 \delta_{\sigma}-3\right)\left(3 \delta_{\sigma}-2 s^{2}+2\right)^{2}}\left[3240 \delta_{\sigma}^{8}-18 \delta_{\sigma}^{7}\left(60 m^{2}+518 s^{2}+177\right)\right. \\
& +6 \delta_{\sigma}^{6}\left(3 m^{2}\left(178 s^{2}+83\right)+1862 s^{4}+2044 s^{2}-1251\right)-24 m^{2} s^{4}\left(s^{2}-1\right)^{2}\left(8 s^{4}+11 s^{2}-10\right) \\
& +\delta_{\sigma}^{5}\left(-6 m^{2}\left(922 s^{4}+574 s^{2}-263\right)-7564 s^{6}-16162 s^{4}+15359 s^{2}+60\right) \\
& +\delta_{\sigma}^{4}\left(m^{2}\left(6580 s^{6}+510 s^{4}-2472 s^{2}-352\right)+3460 s^{8}+8476 s^{6}-9133 s^{4}-4781 s^{2}+3112\right) \\
& +4 s^{2} \delta_{\sigma}^{2}\left(m^{2}\left(394 s^{8}-655 s^{6}-1101 s^{4}+1538 s^{2}-257\right)+\left(s^{2}-1\right)^{2}\left(40 s^{6}+75 s^{4}+126 s^{2}-133\right)\right) \\
& -4 m^{2} s^{4}\left(56 s^{8}-287 s^{6}-222 s^{4}+919 s^{2}-466\right) \delta_{\sigma} \\
& -2 \delta_{\sigma}^{3}\left(m^{2}\left(2262 s^{8}-1412 s^{6}-2328 s^{4}+879 s^{2}+140\right)+540 s^{10}+706 s^{8}-577 s^{6}-2817 s^{4}\right. \\
& \left.\left.+2588 s^{2}-440\right)\right]+\frac{3 a^{3} m}{2\left(\delta_{\sigma}-2\right) \delta_{\sigma}^{3}\left(4 \delta_{\sigma}-3\right)\left(3 \delta_{\sigma}-2 s^{2}+2\right)^{2}}\left[126 \delta_{\sigma}^{7}+6 \delta_{\sigma}^{6}\left(45 m^{2}+68 s^{2}-32\right)\right. \\
& -72 m^{2} s^{4}\left(s^{6}-3 s^{2}+2\right)-\delta_{\sigma}^{5}\left(54 m^{2}\left(14 s^{2}+9\right)+994 s^{4}+301 s^{2}+208\right) \\
& +\delta_{\sigma}^{4}\left(6 m^{2}\left(199 s^{4}+166 s^{2}-32\right)+780 s^{6}+1127 s^{4}-901 s^{2}+128\right) \\
& +12 s^{2} \delta_{\sigma}^{2}\left(m^{2}\left(41 s^{6}-3 s^{4}-90 s^{2}+25\right)+\left(s^{2}-1\right)^{2}\left(5 s^{4}+14 s^{2}+17\right)\right) \\
& -12 m^{2} s^{4}\left(7 s^{6}-18 s^{4}-39 s^{2}+50\right) \delta_{\sigma} \\
& \left.-6 \delta_{\sigma}^{3}\left(m^{2}\left(186 s^{6}+80 s^{4}-67 s^{2}-28\right)+54 s^{8}+89 s^{6}-107 s^{4}-20 s^{2}-16\right)\right]+\mathcal{O}\left(a^{4}\right) .
\end{aligned}
$$

We give the coefficient $\bar{\omega}_{2}$ separately for each different value of the spin $s$. For $s=0$ :

$$
\begin{aligned}
& \bar{\omega}_{2}=\frac{9}{8} \sqrt{3} a\left(8 a^{2}-5\right) m+\frac{1}{32} i\left(465 a^{2}-19\right)(2 N+1)-3 i(2 N+1)\left(-24\left(63 a^{2}+2\right) \delta_{\sigma}^{2}\right. \\
& \left.+8\left(21 a^{2}\left(69 m^{2}-25\right)-4\right) \delta_{\sigma}+3 a^{2}\left(5216 m^{2}-695\right)+51\right) /\left[32\left(4 \delta_{\sigma}-3\right)\left(12 \delta_{\sigma}+17\right)^{2}\right] \\
& +\frac{27(2 N+1)^{2}}{16 \sqrt{3} \zeta^{3}\left(4 \delta_{\sigma}-3\right)\left(12 \delta_{\sigma}+17\right)^{2}}\left\{720\left(75 a^{2}-4\right) \delta_{\sigma}^{4}+12\left(3 a^{2}\left(300 m^{2}+3767\right)-596\right) \delta_{\sigma}^{3}\right. \\
& +2\left(3 a^{2}\left(1818 m^{2}+7267\right)-1054\right) \delta_{\sigma}^{2}+\left(4439-3 a^{2}\left(7122 m^{2}+26665\right)\right) \delta_{\sigma} \\
& \left.-a^{2}\left(22228 m^{2}+31937\right)+1785\right\}+\left\{-10368\left(765 a^{2}-76\right) \delta_{\sigma}^{5}\right. \\
& +432\left(3 a^{2}\left(8760 m^{2}-18341\right)+5236\right) \delta_{\sigma}^{4}+108\left(3 a^{2}\left(91484 m^{2}-49331\right)+12268\right) \delta_{\sigma}^{3} \\
& +6\left(9 a^{2}\left(212862 m^{2}+163295\right)-166930\right) \delta_{\sigma}^{2}+\left(-3 a^{2}\left(5249298 m^{2}-3509465\right)-951175\right) \delta_{\sigma} \\
& \left.-15\left(a^{2}\left(459664 m^{2}-155035\right)+12325\right)\right\} /\left[16 \sqrt{3} \zeta^{3}\left(4 \delta_{\sigma}-3\right)\left(12 \delta_{\sigma}+17\right)^{2}\right]+\mathcal{O}\left(a^{4}\right) .
\end{aligned}
$$


For $s= \pm 1 / 2$ :

$$
\begin{aligned}
& \bar{\omega}_{2}=9 \sqrt{3} a^{3} m-\frac{45}{8} \sqrt{3} a m+\frac{1}{32} i\left(465 a^{2}-19\right)(2 N+1) \\
& -\frac{i(2 N+1)}{256 \delta_{\sigma}^{3}\left(2 \delta_{\sigma}+1\right)^{3}\left(4 \delta_{\sigma}+5\right)}\left\{32\left(135 a^{2}-14\right) \delta_{\sigma}^{6}+48\left(a^{2}\left(214 m^{2}+203\right)-21\right) \delta_{\sigma}^{5}\right. \\
& +\left(a^{2}\left(6336 m^{2}+6954\right)-672\right) \delta_{\sigma}^{4}+\left(a^{2}\left(2121-8238 m^{2}\right)-140\right) \delta_{\sigma}^{3}+27 a^{2}\left(10-317 m^{2}\right) \delta_{\sigma}^{2} \\
& \left.-2646 a^{2} m^{2} \delta_{\sigma}-270 a^{2} m^{2}\right\}+\frac{3 \sqrt{3}(2 N+1)^{2}}{256 \zeta^{3} \delta_{\sigma}^{3}\left(2 \delta_{\sigma}+1\right)^{3}\left(4 \delta_{\sigma}+5\right)}\left\{1920\left(75 a^{2}-4\right) \delta_{\sigma}^{8}\right. \\
& +32\left(75 a^{2}\left(12 m^{2}+175\right)-692\right) \delta_{\sigma}^{7}+8\left(a^{2}\left(6372 m^{2}+55869\right)-2900\right) \delta_{\sigma}^{6} \\
& +6\left(a^{2}\left(6604 m^{2}+36965\right)-1892\right) \delta_{\sigma}^{5}+\left(a^{2}\left(31158 m^{2}+50721\right)-2624\right) \delta_{\sigma}^{4} \\
& \left.+\left(3 a^{2}\left(7927 m^{2}+1254\right)-230\right) \delta_{\sigma}^{3}+135 a^{2}\left(78 m^{2}-1\right) \delta_{\sigma}^{2}+2133 a^{2} m^{2} \delta_{\sigma}+135 a^{2} m^{2}\right\} \\
& +\frac{\sqrt{3}}{256 \zeta \delta_{\sigma}^{3}\left(2 \delta_{\sigma}+1\right)^{3}\left(4 \delta_{\sigma}+5\right)}\left\{-256\left(765 a^{2}-76\right) \delta_{\sigma}^{8}+32\left(15 a^{2}\left(584 m^{2}-1213\right)+1796\right) \delta_{\sigma}^{7}\right. \\
& +48\left(a^{2}\left(15494 m^{2}-13239\right)+1296\right) \delta_{\sigma}^{6}+\left(30 a^{2}\left(21096 m^{2}-10825\right)+31592\right) \delta_{\sigma}^{5} \\
& +\left(9 a^{2}\left(19754 m^{2}-8579\right)+7592\right) \delta_{\sigma}^{4}-3\left(a^{2}\left(5485 m^{2}+2104\right)-230\right) \delta_{\sigma}^{3} \\
& \left.-9 a^{2}\left(1552 m^{2}-15\right) \delta_{\sigma}^{2}-1593 a^{2} m^{2} \delta_{\sigma}-135 a^{2} m^{2}\right\}+\mathcal{O}\left(a^{4}\right) .
\end{aligned}
$$

For $s= \pm 1$ :

$$
\begin{aligned}
& \bar{\omega}_{2}=9 \sqrt{3} a^{3} m-\frac{45}{8} \sqrt{3} a m+\frac{1}{32} i\left(465 a^{2}-19\right)(2 N+1) \\
& +\frac{81 \sqrt{3}\left[(2 N+1)^{2}+1\right]\left(\left(1-41 a^{2}\right) \delta_{\sigma}^{2}+18 a^{2} m^{2}\right)}{16 \zeta^{3} \delta_{\sigma}^{3}\left(4 \delta_{\sigma}-3\right)\left(4 \delta_{\sigma}+3\right)^{2}} \\
& -\frac{i(2 N+1)}{32 \delta_{\sigma}^{3}\left(4 \delta_{\sigma}-3\right)\left(4 \delta_{\sigma}+3\right)^{2}}\left\{-8\left(729 a^{2}-50\right) \delta_{\sigma}^{5}+24\left(a^{2}\left(55 m^{2}-41\right)+8\right) \delta_{\sigma}^{4}\right. \\
& \left.+9\left(a^{2}\left(464 m^{2}+799\right)-25\right) \delta_{\sigma}^{3}-108\left(a^{2}\left(112 m^{2}-41\right)+1\right) \delta_{\sigma}^{2}-11664 a^{2} m^{2} \delta_{\sigma}-1944 a^{2} m^{2}\right\} \\
& +\frac{3 \sqrt{3}(2 N+1)^{2}}{16 \zeta^{3} \delta_{\sigma}\left(4 \delta_{\sigma}-3\right)\left(4 \delta_{\sigma}+3\right)^{2}}\left\{240\left(75 a^{2}-4\right) \delta_{\sigma}^{5}\right. \\
& +4\left(a^{2}\left(900 m^{2}+597\right)-20\right) \delta_{\sigma}^{4}+2\left(9 a^{2}\left(10 m^{2}-737\right)+406\right) \delta_{\sigma}^{3} \\
& \left.+3\left(a^{2}\left(754 m^{2}+901\right)-1\right) \delta_{\sigma}^{2}-9\left(a^{2}\left(1160 m^{2}-509\right)+17\right) \delta_{\sigma}-8640 a^{2} m^{2}\right\} \\
& +\frac{3 \sqrt{3}}{16 \zeta^{3} \delta_{\sigma}\left(4 \delta_{\sigma}-3\right)\left(4 \delta_{\sigma}+3\right)^{2}}\left\{-128\left(765 a^{2}-76\right) \delta_{\sigma}^{6}+16\left(a^{2}\left(8760 m^{2}+603\right)+124\right) \delta_{\sigma}^{5}\right. \\
& -4\left(3 a^{2}\left(2572 m^{2}-6911\right)+2012\right) \delta_{\sigma}^{4}-2\left(3 a^{2}\left(23474 m^{2}+5205\right)+278\right) \delta_{\sigma}^{3} \\
& \left.+3\left(a^{2}\left(31222 m^{2}-7631\right)+467\right) \delta_{\sigma}^{2}+9\left(a^{2}\left(5572 m^{2}+1265\right)-35\right) \delta_{\sigma}-25164 a^{2} m^{2}\right\}+\mathcal{O}\left(a^{4}\right) .
\end{aligned}
$$


For $s= \pm 2$ :

$$
\begin{aligned}
& \bar{\omega}_{2}=9 \sqrt{3} a^{3} m-\frac{45}{8} \sqrt{3} a m+\frac{1}{32} i\left(465 a^{2}-19\right)(2 N+1) \\
& +\frac{i(2 N+1)}{32\left(5-4 \delta_{\sigma}\right)^{2}\left(\delta_{\sigma}-2\right)^{3} \delta_{\sigma}^{3}\left(4 \delta_{\sigma}-3\right)}\left\{-8\left(3105 a^{2}-194\right) \delta_{\sigma}^{8}\right. \\
& +8\left(3 a^{2}\left(703 m^{2}+11631\right)-1744\right) \delta_{\sigma}^{7}-3\left(a^{2}\left(105136 m^{2}+432723\right)-15973\right) \delta_{\sigma}^{6} \\
& +2\left(3 a^{2}\left(292720 m^{2}+532233\right)-39133\right) \delta_{\sigma}^{5} \\
& +\left(60340-36 a^{2}\left(117968 m^{2}+122227\right)\right) \delta_{\sigma}^{4}+24\left(a^{2}\left(192848 m^{2}+135795\right)-725\right) \delta_{\sigma}^{3} \\
& \left.-1152 a^{2}\left(1139 m^{2}+900\right) \delta_{\sigma}^{2}-1486080 a^{2} m^{2} \delta_{\sigma}+1036800 a^{2} m^{2}\right\} \\
& +\frac{3 \sqrt{3}(2 N+1)^{2}}{16 \zeta^{3}\left(5-4 \delta_{\sigma}\right)^{2}\left(\delta_{\sigma}-2\right)^{3} \delta_{\sigma}^{3}\left(4 \delta_{\sigma}-3\right)}\left\{240\left(75 a^{2}-4\right) \delta_{\sigma}^{10}+4\left(15 a^{2}\left(60 m^{2}-3901\right)+3148\right) \delta_{\sigma}^{9}\right. \\
& -2\left(3 a^{2}\left(5298 m^{2}-226909\right)+35482\right) \delta_{\sigma}^{8}-3\left(a^{2}\left(12302 m^{2}+1554911\right)-73607\right) \delta_{\sigma}^{7} \\
& +\left(3 a^{2}\left(395920 m^{2}+3455569\right)-405995\right) \delta_{\sigma}^{6}-2\left(15 a^{2}\left(161384 m^{2}+512431\right)-218393\right) \delta_{\sigma}^{5} \\
& +12\left(a^{2}\left(717768 m^{2}+1242091\right)-20995\right) \delta_{\sigma}^{4}-24\left(a^{2}\left(261416 m^{2}+361395\right)-2475\right) \delta_{\sigma}^{3} \\
& \left.-288 a^{2}\left(4489 m^{2}-8100\right) \delta_{\sigma}^{2}+4898880 a^{2} m^{2} \delta_{\sigma}-2332800 a^{2} m^{2}\right\} \\
& +\frac{3 \sqrt{3}}{16 \zeta^{3}\left(5-4 \delta_{\sigma}\right)^{2}\left(\delta_{\sigma}-2\right)^{3} \delta_{\sigma}^{3}\left(4 \delta_{\sigma}-3\right)}\left\{-128\left(765 a^{2}-76\right) \delta_{\sigma}^{11}\right. \\
& +48\left(5 a^{2}\left(584 m^{2}+6277\right)-2796\right) \delta_{\sigma}^{10}-4\left(3 a^{2}\left(171852 m^{2}+857513\right)-201988\right) \delta_{\sigma}^{9} \\
& +2\left(3 a^{2}\left(2349982 m^{2}+6875683\right)-1385222\right) \delta_{\sigma}^{8}-3\left(5 a^{2}\left(3918450 m^{2}+7190207\right)-1969787\right) \delta_{\sigma}^{7} \\
& +\left(3 a^{2}\left(54007292 m^{2}+64065961\right)-8007761\right) \delta_{\sigma}^{6} \\
& -6\left(a^{2}\left(49556268 m^{2}+39151043\right)-1117417\right) \delta_{\sigma}^{5} \\
& +12\left(a^{2}\left(28901884 m^{2}+15878979\right)-262665\right) \delta_{\sigma}^{4}-72\left(a^{2}\left(3095108 m^{2}+1294785\right)-8775\right) \delta_{\sigma}^{3} \\
& \left.+69984 a^{2}\left(493 m^{2}+300\right) \delta_{\sigma}^{2}+44089920 a^{2} m^{2} \delta_{\sigma}-20995200 a^{2} m^{2}\right\}+\mathcal{O}\left(a^{4}\right) .
\end{aligned}
$$

\section{E Coefficients of the angular eigenvalue expansion}

In this appendix we give the explicit form of the coefficients in the expansion for the eigenvalue in eq. (1.3), expanded for small $\alpha$. As in the previous appendix, we define $\delta_{\sigma} \equiv \ell(\ell+1)$

$$
\begin{aligned}
\lambda_{\omega, 0} \equiv & \delta_{\sigma}-s^{2}+\frac{\alpha^{2}}{\delta_{\sigma}\left(4 \delta_{\sigma}-3\right)}\left[-2 s^{4}\left(\delta_{\sigma}-3 m^{2}\right)+s^{2}\left(\delta_{\sigma}\left(4 m^{2}+1\right)-6 m^{2}\right)\right. \\
& \left.+\delta_{\sigma}\left(\delta_{\sigma}\left(2 \delta_{\sigma}+6 m^{2}+1\right)-4 m^{2}-2\right)\right]+\mathcal{O}\left(\alpha^{4}\right), \\
\lambda_{\omega, 1} \equiv & -2 m\left(1+\frac{s^{2}}{\delta_{\sigma}}\right)-\frac{2 \alpha^{2} m}{\delta_{\sigma}^{3}\left(\delta_{\sigma}-2\right)\left(4 \delta_{\sigma}-3\right)}\left[-2 s^{6}\left(5 \delta_{\sigma}^{2}-\left(7 \delta_{\sigma}+6\right) m^{2}\right)+\delta_{\sigma}\right)^{2} s^{2} \times \\
& \left(3 \delta_{\sigma}\left(2 \delta_{\sigma}-2 m^{2}-7\right)+22 m^{2}+8\right)+\delta_{\sigma}^{3}\left(\delta_{\sigma}-2\right)\left(6 \delta_{\sigma}+2 m^{2}-5\right) \\
& \left.+2 s^{4}\left(\delta_{\sigma}\left(\delta_{\sigma}\left(3 \delta_{\sigma}-5 m^{2}+4\right)-7 m^{2}\right)-6 m^{2}\right)\right]+\mathcal{O}\left(\alpha^{4}\right), \\
\lambda_{\omega, 2} \equiv & \frac{2}{\delta_{\sigma}^{3}\left(4 \delta_{\sigma}-3\right)}\left\{s^{4}\left[\left(5 \delta_{\sigma}+3\right) m^{2}-3 \delta_{\sigma}^{2}\right]+2 \delta_{\sigma}^{2} s^{2}\left(\delta_{\sigma}-3 m^{2}\right)+\delta_{\sigma}^{3}\left(\delta_{\sigma}+m^{2}-1\right)\right\} \\
& +\mathcal{O}\left(\alpha^{2}\right), \\
\lambda_{\omega, 3} \equiv & -\frac{4 m s^{2}}{\delta_{\sigma}^{5}\left(\delta_{\sigma}-2\right)\left(4 \delta_{\sigma}-3\right)}\left(-\delta_{\sigma}^{4}\left(3 \delta_{\sigma}-5 m^{2}-1\right)+2 \delta_{\sigma}^{2} s^{2}\left(5 \delta_{\sigma}^{2}-\left(7 \delta_{\sigma}+6\right) m^{2}\right)\right. \\
& \left.+s^{4}\left(\delta_{\sigma}\left(19 m^{2}-\delta_{\sigma}\left(7 \delta_{\sigma}-9 m^{2}+6\right)\right)+6 m^{2}\right)\right)+\mathcal{O}\left(\alpha^{2}\right) .
\end{aligned}
$$


The expressions for $\lambda_{\omega, 0}$ and $\lambda_{\omega, 1}$ which we provide agree with [11] (which also provide the $\mathcal{O}\left(\alpha^{2}\right)$ term in $\left.\lambda_{\omega, 2}\right)$. The leading-order terms in the expansions for $\lambda_{\omega, 2}$ and $\lambda_{\omega, 3}$ (which are not provided in [11]) are, in fact, just the same as in Kerr, and agree with [22].

Open Access. This article is distributed under the terms of the Creative Commons Attribution License (CC-BY 4.0), which permits any use, distribution and reproduction in any medium, provided the original author(s) and source are credited.

\section{References}

[1] B.F. Whiting, Mode Stability of the Kerr Black Hole, J. Math. Phys. 30 (1989) 1301 [INSPIRE].

[2] LigO ScIEnTific and VIRGo collaborations, Observation of Gravitational Waves from a Binary Black Hole Merger, Phys. Rev. Lett. 116 (2016) 061102 [arXiv:1602.03837] [INSPIRE].

[3] G.T. Horowitz and V.E. Hubeny, Quasinormal modes of AdS black holes and the approach to thermal equilibrium, Phys. Rev. D 62 (2000) 024027 [hep-th/9909056] [INSPIRE].

[4] K.D. Kokkotas and B.G. Schmidt, Quasinormal modes of stars and black holes, Living Rev. Rel. 2 (1999) 2 [gr-qc/9909058] [INSPIRE].

[5] E. Berti, V. Cardoso and A.O. Starinets, Quasinormal modes of black holes and black branes, Class. Quant. Grav. 26 (2009) 163001 [arXiv:0905.2975] [INSPIRE].

[6] S.A. Teukolsky, Perturbations of a rotating black hole. 1. Fundamental equations for gravitational electromagnetic and neutrino field perturbations, Astrophys. J. 185 (1973) 635 [INSPIRE].

[7] O.J.C. Dias, J.E. Santos and M. Stein, Kerr-AdS and its Near-horizon Geometry: Perturbations and the Kerr/CFT Correspondence, JHEP 10 (2012) 182 [arXiv:1208.3322] [INSPIRE].

[8] U. Khanal, Rotating black hole in asymptotic de Sitter space: perturbation of the space-time with spin fields, Phys. Rev. D 28 (1983) 1291 [INSPIRE].

[9] C.M. Chambers and I.G. Moss, Stability of the Cauchy horizon in Kerr-de Sitter space-times, Class. Quant. Grav. 11 (1994) 1035 [gr-qc/9404015] [inSPIRE].

[10] D. Batic and H. Schmid, Heun equation, Teukolsky equation and type-D metrics, J. Math. Phys. 48 (2007) 042502 [gr-qc/0701064] [InSPIRE].

[11] H. Suzuki, E. Takasugi and H. Umetsu, Perturbations of Kerr-de Sitter black hole and Heun's equations, Prog. Theor. Phys. 100 (1998) 491 [gr-qc/9805064] [INSPIRE].

[12] A. Ronveaux and F. Arscott, Heun's differential equations, Oxford University Press (1995).

[13] R. Garnier, Sur des équations différentielles du troisième ordre dont l'intégrale générale est uniforme et sur une classe d'équations nouvelles d'ordre supérieur dont l'intégrale générale à ses points critiques fixes, Annales Sci. Ecole Norm. Sup. 29 (1912) 1.

[14] K. Iwasaki, H. Kimura, S. Shimomura and M. Yoshida, From Gauss to Painlevé: A Modern Theory of Special Functions, in Aspects of Mathematics E, vol. 16, Braunschweig (1991).

[15] A. Litvinov, S. Lukyanov, N. Nekrasov and A. Zamolodchikov, Classical Conformal Blocks and Painleve VI, JHEP 07 (2014) 144 [arXiv:1309.4700] [INSPIRE]. 
[16] M. Lencsés and F. Novaes, Classical Conformal Blocks and Accessory Parameters from Isomonodromic Deformations, JHEP 04 (2018) 096 [arXiv: 1709.03476] [INSPIRE].

[17] T. Anselmo, R. Nelson, B.C. da Cunha and D.G. Crowdy, Accessory parameters in conformal mapping: exploiting the isomonodromic tau function for Painlevé VI, Proc. Roy. Soc. Lond. A 474 (2018) 20180080.

[18] P. Menotti, On the monodromy problem for the four-punctured sphere, J. Phys. A 47 (2014) 415201 [arXiv: 1401.2409] [inSPIRE].

[19] L. Hollands and O. Kidwai, Higher length-twist coordinates, generalized Heun's opers and twisted superpotentials, arXiv:1710.04438 [INSPIRE].

[20] D. Anninos and T. Anous, A de Sitter Hoedown, JHEP 08 (2010) 131 [arXiv:1002.1717] [INSPIRE].

[21] S. Yoshida, N. Uchikata and T. Futamase, Quasinormal modes of Kerr-de Sitter black holes, Phys. Rev. D 81 (2010) 044005 [inSPIRE].

[22] E. Berti, V. Cardoso and M. Casals, Eigenvalues and eigenfunctions of spin-weighted spheroidal harmonics in four and higher dimensions, Phys. Rev. D 73 (2006) 024013 [Erratum ibid. D 73 (2006) 109902] [gr-qc/0511111] [INSPIRE].

[23] E.W. Leaver, An Analytic representation for the quasi normal modes of Kerr black holes, Proc. Roy. Soc. Lond. A 402 (1985) 285 [INSPIRE].

[24] E.W. Leaver, Solutions to a generalized spheroidal wave equation: Teukolsky's equations in general relativity, and the two-center problem in molecular quantum mechanics, J. Math. Phys. 27 (1986) 1238.

[25] O.J. Tattersall, Kerr-(anti-)de Sitter black holes: Perturbations and quasinormal modes in the slow rotation limit, Phys. Rev. D 98 (2018) 104013 [arXiv: 1808.10758] [INSPIRE].

[26] S. Dyatlov, Asymptotic distribution of quasi-normal modes for Kerr-de Sitter black holes, Annales Henri Poincaré 13 (2012) 1101 [arXiv:1101.1260] [INSPIRE].

[27] O.J.C. Dias, F.C. Eperon, H.S. Reall and J.E. Santos, Strong cosmic censorship in de Sitter space, Phys. Rev. D 97 (2018) 104060 [arXiv:1801.09694] [INSPIRE].

[28] F. Novaes and B. Carneiro da Cunha, Isomonodromy, Painlevé transcendents and scattering off of black holes, JHEP 07 (2014) 132 [arXiv: 1404.5188] [INSPIRE].

[29] B. Carter, Black holes (les astres occlus), in Black Hole Equilibrium States, Gordon and Breach Science Publishers, NY (1973).

[30] F. Mellor and I. Moss, Stability of Black Holes in de Sitter Space, Phys. Rev. D 41 (1990) 403 [INSPIRE].

[31] S. Akcay and R.A. Matzner, Kerr-de Sitter Universe, Class. Quant. Grav. 28 (2011) 085012 [arXiv: 1011.0479] [INSPIRE].

[32] I.S. Booth and R.B. Mann, Cosmological pair production of charged and rotating black holes, Nucl. Phys. B 539 (1999) 267 [gr-qc/9806056] [InSPIRE].

[33] P. Hintz and A. Vasy, The global non-linear stability of the Kerr-de Sitter family of black holes, arXiv: 1606.04014 [INSPIRE].

[34] A. Castro, J.M. Lapan, A. Maloney and M.J. Rodriguez, Black Hole Scattering from Monodromy, Class. Quant. Grav. 30 (2013) 165005 [arXiv:1304.3781] [INSPIRE]. 
[35] B. Carneiro da Cunha and F. Novaes, Kerr-de Sitter greybody factors via isomonodromy, Phys. Rev. D 93 (2016) 024045 [arXiv: 1508. 04046] [InSPIRE].

[36] S.Y. Slavyanov and W. Lay, Special Functions: A Unified Theory Based on Singularities, Oxford University Press, U.S.A. (2000).

[37] B. Carneiro da Cunha and F. Novaes, Kerr Scattering Coefficients via Isomonodromy, JHEP 11 (2015) 144 [arXiv:1506.06588] [INSPIRE].

[38] A.M. Ghezelbash and R.B. Mann, Entropy and mass bounds of Kerr-de Sitter spacetimes, Phys. Rev. D 72 (2005) 064024 [hep-th/0412300] [INSPIRE].

[39] S.L. Detweiler, Black holes and gravitational waves. III. The resonant frequencies of rotating holes, Astrophys. J. 239 (1980) 292 [INSPIRE].

[40] T. Harmark, J. Natario and R. Schiappa, Greybody Factors for d-Dimensional Black Holes, Adv. Theor. Math. Phys. 14 (2010) 727 [arXiv:0708.0017] [INSPIRE].

[41] H. Yang, A. Zimmerman, A. Zenginoğlu, F. Zhang, E. Berti and Y. Chen, Quasinormal modes of nearly extremal Kerr spacetimes: spectrum bifurcation and power-law ringdown, Phys. Rev. D 88 (2013) 044047 [arXiv: 1307.8086] [INSPIRE].

[42] A. Castro, A. Maloney and A. Strominger, Hidden Conformal Symmetry of the Kerr Black Hole, Phys. Rev. D 82 (2010) 024008 [arXiv: 1004.0996] [inSPIRE].

[43] L.C.B. Crispino, A. Higuchi, E.S. Oliveira and J.V. Rocha, Greybody factors for nonminimally coupled scalar fields in Schwarzschild-de Sitter spacetime, Phys. Rev. D 87 (2013) 104034 [arXiv: 1304.0467] [INSPIRE].

[44] S. Mano, H. Suzuki and E. Takasugi, Analytic solutions of the Teukolsky equation and their low frequency expansions, Prog. Theor. Phys. 95 (1996) 1079 [gr-qc/9603020] [INSPIRE].

[45] M. Sasaki and H. Tagoshi, Analytic black hole perturbation approach to gravitational radiation, Living Rev. Rel. 6 (2003) 6 [gr-qc/0306120] [INSPIRE].

[46] M. Casals and P. Zimmerman, Perturbations of Extremal Kerr Spacetime: Analytic Framework and Late-time Tails, arXiv:1801.05830 [INSPIRE].

[47] H. Suzuki, E. Takasugi and H. Umetsu, Analytic solutions of Teukolsky equation in Kerr-de Sitter and Kerr-Newman-de Sitter geometries, Prog. Theor. Phys. 102 (1999) 253 [gr-qc/9905040] [INSPIRE].

[48] H. Suzuki, E. Takasugi and H. Umetsu, Absorption rate of the Kerr-de Sitter black hole and the Kerr-Newman-de Sitter black hole, Prog. Theor. Phys. 103 (2000) 723 [gr-qc/9911079] [INSPIRE].

[49] L. Motl and A. Neitzke, Asymptotic black hole quasinormal frequencies, Adv. Theor. Math. Phys. 7 (2003) 307 [hep-th/0301173] [INSPIRE].

[50] M. Casals and A.C. Ottewill, Spin-1 quasinormal frequencies in Schwarzschild spacetime for large overtone number, Phys. Rev. D 97 (2018) 024048 [arXiv: 1606.03423] [INSPIRE].

[51] T. Tachizawa and K.-i. Maeda, Superradiance in the kerr-de sitter space-time, Phys. Lett. A $172(1993) 325$.

[52] M. Casals, C. Kavanagh and A.C. Ottewill, High-order late-time tail in a Kerr spacetime, Phys. Rev. D 94 (2016) 124053 [arXiv: 1608.05392] [INSPIRE]. 
[53] B.E. Barrowes, K. O'Neill, G.T.M. and J.A. Kong, On the Asymptotic Expansion of the Spheroidal Wave Function and Its Eigenvalues for Complex Size Parameter, Stud. Appl. Math. 113 (2004) 271.

[54] E.S.C. Ching, P.T. Leung, W.M. Suen and K. Young, Wave propagation in gravitational systems: Late time behavior, Phys. Rev. D 52 (1995) 2118 [gr-qc/9507035] [INSPIRE].

[55] K. Glampedakis and N. Andersson, Late time dynamics of rapidly rotating black holes, Phys. Rev. D 64 (2001) 104021 [gr-qc/0103054] [INSPIRE].

[56] L. Barack and A. Ori, Late time decay of scalar perturbations outside rotating black holes, Phys. Rev. Lett. 82 (1999) 4388 [gr-qc/9902082] [inSPIRE].

[57] S. Hod, The Radiative tail of realistic gravitational collapse, Phys. Rev. Lett. 84 (2000) 10 [gr-qc/9907096] [INSPIRE].

[58] M. Casals, S.E. Gralla and P. Zimmerman, Horizon Instability of Extremal Kerr Black Holes: Nonaxisymmetric Modes and Enhanced Growth Rate, Phys. Rev. D 94 (2016) 064003 [arXiv: 1606.08505] [INSPIRE].

[59] S.E. Gralla and P. Zimmerman, Critical Exponents of Extremal Kerr Perturbations, Class. Quant. Grav. 35 (2018) 095002 [arXiv:1711.00855] [INSPIRE].

[60] S. Aretakis, Horizon Instability of Extremal Black Holes, Adv. Theor. Math. Phys. 19 (2015) 507 [arXiv: 1206.6598] [INSPIRE].

[61] S. Hod, Slow relaxation of rapidly rotating black holes, Phys. Rev. D 78 (2008) 084035 [arXiv: 0811.3806] [INSPIRE].

[62] D. Anninos and T. Hartman, Holography at an Extremal de Sitter Horizon, JHEP 03 (2010) 096 [arXiv:0910.4587] [INSPIRE].

[63] NIST Digital Library of Mathematical Functions, Release 1.0.5, 2012-10-01 [http://dlmf.nist.gov/].

[64] V. Cardoso and J.P.S. Lemos, Quasinormal modes of the near extremal Schwarzschild-de Sitter black hole, Phys. Rev. D 67 (2003) 084020 [gr-qc/0301078] [INSPIRE].

[65] M. Jimbo, T. Miwa and A.K. Ueno, Monodromy Preserving Deformation of Linear Ordinary Differential Equations With Rational Coefficients. I, Physica D 2 (1981) 306.

[66] M. Jimbo and T. Miwa, Monodromy Preserving Deformation of Linear Ordinary Differential Equations with Rational Coefficients. II, Physica D 2 (1981) 407.

[67] M. Jimbo and T. Miwa, Monodromy Preserving Deformation of Linear Ordinary Differential Equations with Rational Coefficients. III, Physica D 4 (1981) 26.

[68] M. Casals and C. Marinho, in preparation.

[69] P. Menotti, Classical conformal blocks, Mod. Phys. Lett. A 31 (2016) 1650159 [arXiv: 1601.04457] [INSPIRE].

[70] N. Iorgov, O. Lisovyy and Yu. Tykhyy, Painlevé VI connection problem and monodromy of $c=1$ conformal blocks, JHEP 12 (2013) 029 [arXiv:1308.4092] [INSPIRE].

[71] J.B. Amado, B. Carneiro da Cunha and E. Pallante, On the Kerr-AdS/CFT correspondence, JHEP 08 (2017) 094 [arXiv:1702.01016] [INSPIRE]. 
[72] J. Barragán-Amado, B. Carneiro Da Cunha and E. Pallante, Scalar quasinormal modes of Kerr- $A d S_{5}$, arXiv: 1812.08921 [INSPIRE].

[73] O. Gamayun, N. Iorgov and O. Lisovyy, How instanton combinatorics solves Painlevé VI, V and IIIs, J. Phys. A 46 (2013) 335203 [arXiv:1302.1832] [InSPIRE].

[74] O. Lisovyy, H. Nagoya and J. Roussillon, Irregular conformal blocks and connection formulae for Painlevé V functions, J. Math. Phys. 59 (2018) 091409 [arXiv: 1806.08344] [InSPIRE].

[75] N. Iorgov, O. Lisovyy and J. Teschner, Isomonodromic tau-functions from Liouville conformal blocks, Commun. Math. Phys. 336 (2015) 671 [arXiv:1401.6104] [InSPIRE].

[76] P. Gavrylenko and O. Lisovyy, Fredholm Determinant and Nekrasov Sum Representations of Isomonodromic Tau Functions, Commun. Math. Phys. 363 (2018) 1 [arXiv:1608.00958] [INSPIRE].

[77] V. Frolov, P. Krtous and D. Kubiznak, Black holes, hidden symmetries and complete integrability, Living Rev. Rel. 20 (2017) 6 [arXiv:1705.05482] [INSPIRE].

[78] M. Guica, T. Hartman, W. Song and A. Strominger, The Kerr/CFT Correspondence, Phys. Rev. D 80 (2009) 124008 [arXiv:0809.4266] [INSPIRE].

[79] G. Compère, The Kerr/CFT correspondence and its extensions, Living Rev. Rel. 15 (2012) 11 [arXiv: 1203.3561] [inSPIRE].

[80] B. Carneiro da Cunha and M. Guica, Exploring the BTZ bulk with boundary conformal blocks, arXiv: 1604.07383 [INSPIRE].

[81] M. Piatek and A.R. Pietrykowski, Solving Heun's equation using conformal blocks, Nucl. Phys. B 938 (2019) 543 [arXiv:1708.06135] [InSPIRE].

[82] D. Braak, Integrability of the rabi model, Phys. Rev. Lett. 107 (2011) 100401 [arXiv: 1103.2461].

[83] B. Carneiro da Cunha, M.C. de Almeida and A.R. de Queiroz, On the Existence of Monodromies for the Rabi model, arXiv: 1508.01342 [INSPIRE].

[84] O. Gamayun, N. Iorgov and O. Lisovyy, Conformal field theory of Painlevé VI, JHEP 10 (2012) 038 [Erratum ibid. 10 (2012) 183] [arXiv: 1207.0787] [INSPIRE].

[85] L.F. Alday, D. Gaiotto and Y. Tachikawa, Liouville Correlation Functions from Four-dimensional Gauge Theories, Lett. Math. Phys. 91 (2010) 167 [arXiv:0906.3219] [INSPIRE]. 